\title{
Photon orbital angular momentum in astronomy
}

\author{
N. M. Elias II ${ }^{1,2}$ \\ 1 Zentrum für Astronomie der Universität Heidelberg, Landessternwarte, Königstuhl 12, 69117 Heidelberg, Germany \\ 2 Max-Planck-Institut für Astronomie; Königstuhl 17, 69117 Heidelberg, Germany \\ e-mail: n.elias@lsw.uni-heidelberg.de
}

Received 17 March 2008 / Accepted 7 October 2008

\begin{abstract}
Context. Photon orbital angular momentum (POAM) has been created in the laboratory, yet it is still relatively unknown. How does POAM manifest itself in astronomy? Are there any applications for measuring astrophysical POAM?

Aims. In this paper, I 1) explain POAM in an astronomical context; 2) define the POAM observables for astronomy; 3) create generic systems-based calculi that describe how POAM propagates from celestial sphere to detector; 4) use the calculi with several astronomical instruments as examples of their utility; 5) demonstrate an application for astrophysical POAM measurements; and 6) relate POAM to existing astronomical instruments and concepts.

Methods. Electric fields are expanded into azimuthal Fourier components, and the intensities are expanded into correlations or rancors. The source electric fields are spatially incoherent. In the systems-based calculi, the inputs are located on the celestial sphere, the system is represented by propagation through free space and instrument, and the outputs are located in a specific plane. The diffraction and point-spread function expansions are very generic and can be used with any type of instrument. I employ these examples to demonstrate the calculi (in order of increasing difficulty): free space, single telescopes, interferometers, coronagraphs, and rancorimeters.

Results. The azimuthal Fourier components of the electric field correspond to POAM vortex states. Rancors contain less information than correlations, yet they are easier to measure and can be used in many applications. Propagation through an aberrated telescope applies external torque, which may be expressed in terms of Zernike polynomials. I prove that a sectored phase mask in a focal-plane coronagraph applies torque to the low-order states, producing a null. Also, I prove that a Michelson interferometer is inherently capable of filtering POAM; e.g., tracking $180^{\circ}$ from the central fringe eliminates even states, producing a null. A limited rancorimeter can be created by placing a focal-plane wedge mask in a coronagraph. The resulting rancors can be used to perform super-Rayleigh observations of unresolved unresolved objects, such as binary stars. There are three types of source POAM: intrinsic, structure, and pointing. Instrumental POAM, which must be calibrated, includes optical aberrations and atmospheric turbulence.

Conclusions. This paper represents the starting point for future research: 1) making a priori predictions about the intrinsic POAM of astronomical sources; 2) designing ground- and space-based POAM-measuring instruments; 3 ) understanding existing instruments in terms of POAM; 4) minimizing the effects of random noise on POAM; and 5) calibrating all types of instrumental POAM.
\end{abstract}

Key words. instrumentation: miscellaneous - methods: analytical - methods: observational - techniques: miscellaneous

\section{Introduction}

Electromagnetic fields may be described in terms of intensity, wavelength, coherence, and angular momentum. Angular momentum consists of two distinct parts, spin and orbital (Marcuse 1980; Heitler 1984). Spin angular momentum is classically represented by photons rotating about their own axis. Orbital angular momentum, on the other hand, is somewhat of a misnomer because under normal circumstances photons do not orbit about a fixed point in space. The canonical view of orbital angular momentum shows the photon Poynting vector precessing about the nominal propagation direction, i.e., spiral wavefronts. Spin and orbital angular momentum are different in another fundamental way, namely that the former consists of two orthogonal states while the latter consists of an infinite number of orthogonal states.

\subsection{History}

Because the concept of photon orbital angular momentum (POAM) is difficult to visualize and understand, it is merely mentioned (Jackson 1975; Mandel \& Wolf 1995) and even neglected (Reitz et al. 1980; Griffiths 1981) in elementary electromagnetics textbooks. Furthermore, a search of the scientific literature shows that the number of POAM articles is smaller than the number of photon spin angular momentum (PSAM, more commonly known as polarization) articles. In recent years, however, the amount of theoretical and applied POAM research has been increasing steadily.

Allen et al. (1992) recognized that light beams with a helical phase dependence carry quantized POAM that is independent of PSAM. Tamm \& Weiss (1990) have directly generated Laguerre-Gaussian (LG) laser beams, which exhibit these helical wavefronts. POAM may be generated with spatial light modulators (SLMs), which are pixelated liquid-crystal devices (Bazhenov et al. 1990). Leach et al. (2002) have developed a technique, employing a modified Mach-Zehnder interferometer, to measure the POAM state of 
individual photons. All of this progress has been summarized by Padgett et al. (2004). Molina-Terriza et al. (2007) also published a general review of various aspects of POAM.

Harwit (2003) described astrophysical processes that generate POAM, including light scattering off inhomogeneities in the environments surrounding energetic sources (e.g. masers, pulsars, and quasars), and photons scattering off rotating black holes. In addition, he mentions the advantages of POAM for SETI, namely multiple-bit encoding per photon, entanglement, and the lack of natural high-POAM sources. Sjöholm \& Palmer (2007) and Thidé et al. (2007) have also conducted POAM simulations at radio wavelengths, which may be relevant for astronomical applications.

\subsection{Applications}

So, what are the applications for POAM in astronomy?

The most obvious application is the characterization of POAM and how it relates to underlying physical processes. Harwit (2003) showed that exotic astronomical sources generate electric fields with non-zero POAM. Do common astronomical sources also generate non-zero POAM? If so, what produces it? Can it be exploited to obtain valuable information?

I found these questions difficult to answer because laboratory and astrophysical POAM differ from each other in fundamental ways. Laboratory POAM sources typically consist of individual vortices and are created by lasers and dedicated optics. Astrophysical POAM sources, on the other hand, could contain individual vortices, but it is more likely that they contain multiple vortices due to large-scale structure. In addition, the electric fields produced by laboratory sources are generally spatially coherent, while the electric fields arising from the celestial sphere are generally spatially incoherent. These distinctions are important and must be taken into account.

The focus of this paper has shifted significantly with time. I had originally wanted to investigate POAM generated by astronomical sources. I do invest some effort on that topic, but I decided to leave these detailed analyses for the future. I found that understanding POAM observables and how they propagate is a prerequisite for understanding astrophysical POAM mechanisms as well as advanced topics like instrument design, random error minimization, and systematic error calibration. To that end, I create new observables and propagation calculi and present several important examples of how they are used, including a practical application.

\subsection{Overview}

In Sect. 2, I describe astronomical POAM in terms of a semi-classical/semi-quantum model that is suitable for astronomy. The electric fields are analogous to quantum wavefunctions, while the intensities are analogous to probabilities. Vortices are helical wavefronts with quantized POAM that are independent of coordinate system origin.

In Sect. 3, I expand arbitrary spatially incoherent electric fields on the celestial sphere in terms of azimuthal Fourier series. Each component represents a single POAM state. The intensity can be expanded in terms of correlations of the states. Correlations between different states are called orbital polarization, analogous to PSAM spin polarization. The intensity can also be expanded in terms of its own azimuthal Fourier series. Each term is called a rancor, which is equivalent to the sum of correlated states with the same rancor index and related to orbital polarization. Also, I derive the POAM expectation value in terms of integrated probabilities.

In Sect. 4, I create two calculi, starting with the electric field and intensity overlap integrals, that can be used to model POAM propagation from celestial sphere through free space and instrument to detector. In the following five sections, I use these calculi in conjunction with astronomically relevant examples (in order of increasing difficulty) to demonstrate their utility: free space (Sect. 5), single telescope (Sect. 6), focal-plane coronagraph (Sect. 7), Michelson interferometer (Sect. 8), and limited rancorimeter (Sect. 9). Propagation through free space and an unaberrated telescope applies no external torque to the electric field. Coronagraphs apply torque to (modulate) the zero-order state of the central star, producing a central null. Michelson interferometers filter states according to the baseline and delay, e.g., single-Bracewell interferometers track $180^{\circ}$ from the central fringe to eliminate even states and produce a null. I convert the coronagraph to a limited rancorimeter by replacing the focal-plane phase mask with a rotating opaque wedge mask. It measures only rancors, yet it can perform tasks such as super-Rayleigh modeling of an unresolved binary star.

In Sect. 10, I define a natural zero-POAM calibration source: an unresolved or partially resolved star at the field-of-view (FOV) center. Several simple POAM sources are also discussed, which are used to describe instrinsic, structure, and pointing POAM. Structure and pointing POAM are related to each other. In Sect. 11, I derive general expressions for the responses of aberrated systems that lead to instrumental POAM, and show that Zernike polynomials may be written in terms of POAM expansions (and vice versa). Instrumental POAM (due to optical aberrations and atmospheric turbulence) is analogous to instrumental PSAM because they both must be calibrated for meaningful scientific results.

\section{Simple POAM model}

In astronomy, treating POAM with pure quantum mechanics is not necessary most of the time. Gori et al. (1998) presented a simplified semi-classical/semi-quantum POAM model, drawing on formalisms from both the classical and quantum regimes. In this section, I generalize their approach.

Using the first-order paraxial approximation, the POAM expectation value depends on the azimuthal derivative of the electric field as well as the intensity in the plane of interest. Vortex electric fields represent pure POAM states with a constant POAM expectation value that is independent of coordinate system origin. The mathematics in other sections of this paper use the formulae in this section, replacing the vector $\overrightarrow{\mathbf{H}}$ with vectors from other planes. 


\subsection{The semi-classical/semi-quantum regime}

Consider an ensemble of photons traveling perpendicularly through a plane along the $Z$ axis. The plane can represent the celestial sphere (locally, a plane), the observation plane (containing the entrance pupil of the instrument), the instrument image plane, etc. I assume that all of the photons have the same polarization, so the PSAM nature of the radiation can be ignored. The scalar spatial/temporal wave function on the plane is

$\Psi(\overrightarrow{\mathbf{H}} ; t)=|\Psi(\overrightarrow{\mathbf{H}} ; t)| \mathrm{e}^{j \Phi(\overrightarrow{\mathbf{H}} ; t)}$,

where $\overrightarrow{\mathbf{H}}=\left(H_{x}, H_{y}\right)=(H \cos \epsilon, H \sin \epsilon)$ is the vector in the plane, $H_{x}$ and $H_{y}$ are the Cartesian coordinates, $H$ is the radial coordinate in the plane, $\epsilon$ is the azimuthal coordinate in the plane, and $t$ is the time. It can be divided into a modulus function

$|\Psi(\overrightarrow{\mathbf{H}} ; t)|=\sqrt{\operatorname{Re}^{2}[\Psi(\overrightarrow{\mathbf{H}} ; t)]+\operatorname{Im}^{2}[\Psi(\overrightarrow{\mathbf{H}} ; t)]}$

and an aberration function

$\Phi(\overrightarrow{\mathbf{H}} ; t)=\tan ^{-1}\left[\frac{\operatorname{Im} \Psi(\overrightarrow{\mathbf{H}} ; t)}{\operatorname{Re} \Psi(\overrightarrow{\mathbf{H}} ; t)}\right]$.

This wave function may be employed to represent one or more photons.

The spatial probability density is proportional to the time average of the squared magnitude of the wave function,

$p(\overrightarrow{\mathbf{H}}) \propto\left\langle\frac{1}{2}|\Psi(\overrightarrow{\mathbf{H}} ; t)|^{2}\right\rangle$,

where $\langle\cdot\rangle$ denotes the time average ${ }^{2}$. It should be normalized such that the integral over the relevant region in the plane is unity. The wave function is proportional to the electric field

$\Psi(\overrightarrow{\mathbf{H}} ; t) \propto E(\overrightarrow{\mathbf{H}} ; t)=|E(\overrightarrow{\mathbf{H}} ; t)| \mathrm{e}^{j \Phi(\overrightarrow{\mathbf{H}} ; t)}$,

which means that the spatial probability density is proportional to the intensity

$p(\overrightarrow{\mathbf{H}}) \propto I(\overrightarrow{\mathbf{H}})=\left\langle\frac{1}{2}|E(\overrightarrow{\mathbf{H}} ; t)|^{2}\right\rangle$

Therefore, the normalized probability density is the intensity divided by the intensity integrated over a given region in the plane, or

$p(\overrightarrow{\mathbf{H}})=\frac{I(\overrightarrow{\mathbf{H}})}{\int \mathrm{d}^{2} H I(\overrightarrow{\mathbf{H}})}=\frac{I(\overrightarrow{\mathbf{H}})}{I^{\text {plane }}}$,

where $\mathrm{d}^{2} H=\mathrm{d} H_{x} \mathrm{~d} H_{y}=\mathrm{d} H H \mathrm{~d} \epsilon$ is the differential area element.

Using the first-order paraxial approximation, the time average POAM per photon about the $Z$ axis is

$L_{Z}(\overrightarrow{\mathbf{H}})=\left\langle L_{Z}(\overrightarrow{\mathbf{H}} ; t)\right\rangle=h\left[H_{x} \kappa_{H_{y}}(\overrightarrow{\mathbf{H}})-H_{y} \kappa_{H_{x}}(\overrightarrow{\mathbf{H}})\right]=\hbar\left[H_{x} \frac{\partial \Phi(\overrightarrow{\mathbf{H}})}{\partial H_{y}}-H_{y} \frac{\partial \Phi(\overrightarrow{\mathbf{H}})}{\partial H_{x}}\right]=\hbar \frac{\partial \Phi(\overrightarrow{\mathbf{H}})}{\partial \epsilon}$

where $h$ is the Planck constant, $\hbar=h / 2 \pi$ is the reduced Planck constant,

$\kappa_{H_{x}}(\overrightarrow{\mathbf{H}})=\left\langle\kappa_{H_{x}}(\overrightarrow{\mathbf{H}} ; t)\right\rangle=\frac{1}{2 \pi} \frac{\partial \Phi(\overrightarrow{\mathbf{H}})}{\partial H_{x}}=\left\langle\frac{1}{2 \pi} \frac{\partial \Phi(\overrightarrow{\mathbf{H}} ; t)}{\partial H_{x}}\right\rangle$

and

$\kappa_{H_{y}}(\overrightarrow{\mathbf{H}})=\left\langle\kappa_{H_{y}}(\overrightarrow{\mathbf{H}} ; t)\right\rangle=\frac{1}{2 \pi} \frac{\partial \Phi(\overrightarrow{\mathbf{H}})}{\partial H_{y}}=\left\langle\frac{1}{2 \pi} \frac{\partial \Phi(\overrightarrow{\mathbf{H}} ; t)}{\partial H_{y}}\right\rangle$

are the transverse wavenumbers, and

$\frac{\partial \Phi(\overrightarrow{\mathbf{H}})}{\partial \epsilon}=-\frac{1}{I(\overrightarrow{\mathbf{H}})} \operatorname{Im}\left\langle\frac{1}{2} E(\overrightarrow{\mathbf{H}} ; t) \frac{\partial E^{*}(\overrightarrow{\mathbf{H}} ; t)}{\partial \epsilon}\right\rangle$

${ }^{2}$ I leave the length of the time average arbitrary, because it depends on the photon rate, the amount of atmospheric turbulence, detector statistics, and the specific application. 
is the azimuthal derivative of the aberration function derived in Appendix A. Equation (3a) can be converted to its classical form when $h \kappa_{H_{x}} \rightarrow p_{H_{x}}$ and $h \kappa_{H_{y}} \rightarrow p_{H_{y}}$ (the linear momenta). The transverse wavenumbers are proportional to the slopes of the aberration function and are constant only across uniform wavefronts. The POAM expectation value per photon is the weighted average over the plane, or

$\hat{L}_{Z}^{\text {plane }}=\int \mathrm{d}^{2} H p(\overrightarrow{\mathbf{H}}) L_{Z}(\overrightarrow{\mathbf{H}})=\hbar \int \mathrm{d}^{2} H p(\overrightarrow{\mathbf{H}}) \frac{\partial \Phi(\overrightarrow{\mathbf{H}})}{\partial \epsilon}$

In real systems, integrations over additional variables may be required (Sects. 4 and 8).

\subsection{Vortices}

In general, $\hat{L}_{Z}^{\text {plane }}$ depends on the coordinate system origin. In Eq. (4), POAM is measured with respect to $\left(H_{x}, H_{y}\right)=(0,0)$. If I shift the reference point by $\left(\Delta H_{x}, \Delta H_{y}\right)$, the POAM in the plane becomes

$\hat{L}_{Z}^{\text {plane, }}=h \int \mathrm{d}^{2} H\left[\left(H_{x}+\Delta H_{x}\right) \kappa_{H_{y}}(\overrightarrow{\mathbf{H}})-\left(H_{y}+\Delta H_{y}\right) \kappa_{H_{x}}(\overrightarrow{\mathbf{H}})\right]=\hat{L}_{Z}^{\text {plane }}+h\left(\Delta H_{x} \hat{\kappa}_{H_{y}}^{\text {plane }}-\Delta H_{y} \hat{\kappa}_{H_{x}}^{\text {plane }}\right)$,

where

$\hat{\kappa}_{H_{x}}^{\text {plane }}=\int \mathrm{d}^{2} H p(\overrightarrow{\mathbf{H}}) \kappa_{H_{x}}(\overrightarrow{\mathbf{H}})$

and

$\hat{\kappa}_{H_{y}}^{\text {plane }}=\int \mathrm{d}^{2} H p(\overrightarrow{\mathbf{H}}) \kappa_{H_{y}}(\overrightarrow{\mathbf{H}})$

are the transverse wavenumber expectation values. A spatially invariant POAM expectation value, or $\hat{L}_{Z}^{\text {plane, }}=\hat{L}_{Z}^{\text {plane }}$, requires $\hat{\kappa}_{H_{x}}^{\text {plane }}=$ $\hat{\kappa}_{H_{y}}^{\text {plane }}=0$ for non-zero $\Delta H_{x}$ and $\Delta H_{y}$.

What kind of electric field yields a spatially invariant POAM expectation value? Consider

$E(\overrightarrow{\mathbf{H}} ; t) \rightarrow E_{m}(H ; t) \mathrm{e}^{j m \epsilon}=\left[\left|E_{m}(H ; t)\right| \mathrm{e}^{j \Theta_{m}(H ; t)}\right] \mathrm{e}^{j m \epsilon}$,

which is called a vortex because of its helical form for each $H$ (Indebetouw 1993). The parameter $m$ must be an integer quantum number, otherwise the helix will be discontinuous in $\epsilon$. The intensity of this vortex is

$I(\overrightarrow{\mathbf{H}})=\left\langle\frac{1}{2}|E(\overrightarrow{\mathbf{H}} ; t)|^{2}\right\rangle \rightarrow I_{m, m}(H)=\left\langle\frac{1}{2}\left|E_{m}(H ; t)\right|^{2}\right\rangle$,

which is independent of $\epsilon$. The modulus and aberration functions are

$|\Psi(\overrightarrow{\mathbf{H}} ; t)| \propto\left|E_{m}(H ; t)\right|$

and

$\Phi(\overrightarrow{\mathbf{H}} ; t)=\Theta_{m}(H ; t)+m \epsilon$

The modulus is also independent of $\epsilon$, and for a given $H$ and $t$ the aberration function is a linear function of $\epsilon$. This aberration function leads to $\hat{\kappa}_{H_{x}}^{\text {plane }}=\hat{\kappa}_{H_{y}}^{\text {plane }}=0$ as well as the fundamental equation

$\hat{L}_{Z}^{\text {plane }}=m \hbar$.

Note that $\hat{L}_{7}^{\text {plane }}$ is independent of coordinate system origin, which means that vortices must be pure POAM states. Laboratory studies of POAM typically employ LG laser modes, which uniquely set the radial dependences of the $E_{m}(H ; t)$ (Tamm \& Weiss 1990). For natural astronomical sources, however, there are an infinite number of possible radial dependences, so the generic form of the $E_{m}(H ; t)$ is preferred here. 


\section{Arbitrary POAM expansions}

In this section, I expand the electric field into an azimuthal Fourier series, where each component represents a POAM state. I express the intensity in terms of correlations of these states. Correlations between different states are called orbital polarization. The intensity can also be expanded into its own azimuthal Fourier series, where each rancor component is equivalent to an infinite sum of correlations. I give estimates for the rms electric field amplitudes and phases. Last, I define the POAM expectation value in terms of an infinite sum of integrated probabilities.

Astronomical electric fields generally do not consist of a single vortex, so how can they be expressed within the POAM paradigm? Since $\epsilon$ is periodic in $2 \pi$, any electric field may be expanded in terms of an azimuthal Fourier series,

$E(\overrightarrow{\mathbf{H}} ; t)=\sum_{m=-\infty}^{\infty} E_{m}(H ; t) \mathrm{e}^{j m \epsilon} \stackrel{\mathcal{F}}{\Leftrightarrow} E_{m}(H ; t)=\frac{1}{2 \pi} \int_{0}^{2 \pi} \mathrm{d} \epsilon \mathrm{e}^{-j m \epsilon} E(\overrightarrow{\mathbf{H}} ; t)$.

I employ the same vector $\overrightarrow{\mathbf{H}}$ as in Sect. 2. Yao et al. (2006) used constants and Kennedy et al. (2002) used LG expansions for the Fourier components, but here they are arbitrary functions of $H$ and $t$. This expansion is just the macroscopic description of the POAM state spectrum in the plane. It is analogous to the Jones vector,

$\overrightarrow{\mathbf{E}}(\overrightarrow{\mathbf{H}} ; t)=E_{H_{x}}(\overrightarrow{\mathbf{H}} ; t) \hat{\mathbf{H}}_{x}+E_{H_{y}}(\overrightarrow{\mathbf{H}} ; t) \hat{\mathbf{H}}_{y} \stackrel{\mathcal{J}}{\Leftrightarrow}\left\{\begin{array}{l}E_{H_{x}}(\overrightarrow{\mathbf{H}} ; t)=\hat{\mathbf{H}}_{x} \cdot \overrightarrow{\mathbf{E}}(\overrightarrow{\mathbf{H}} ; t) \\ E_{H_{y}}(\overrightarrow{\mathbf{H}} ; t)=\hat{\mathbf{H}} y \cdot \overrightarrow{\mathbf{E}}(\overrightarrow{\mathbf{H}} ; t)\end{array}\right.$,

which is the macroscopic description for PSAM.

The intensity, expressed in terms of the states in Eq. (9a), is

$I(\vec{H})=\sum_{m=-\infty}^{\infty} \sum_{n=-\infty}^{\infty} I_{m, n}(H) \mathrm{e}^{j(m-n) \epsilon}=\sum_{m=-\infty}^{\infty} I_{m, m}(H)+2 \operatorname{Re} \sum_{m=-\infty}^{\infty} \sum_{n=m+1}^{\infty} I_{m, n}(H) \mathrm{e}^{j(m-n) \epsilon}$,

where the

$I_{m, n}(H)=I_{n, m}^{*}(H)=\left\langle\frac{1}{2} E_{m}(H ; t) E_{n}^{*}(H ; t)\right\rangle$

are the components of the correlation spectrum. The sums consist of two types of terms, autocorrelated $(m=n)$ and cross-correlated $(m \neq n)$. The cross-correlated terms contain orbital polarization, or rancor ${ }^{2}$. These quantities are analogous to PSAM spin polarization. If all of the cross correlations are zero, the radiation is unrancored.

The rms states of any source can be determined directly from its correlations. The rms magnitude of each state $m$ comes from the $(m, m)$ autocorrelation

$\bar{E}_{m}(H)=\sqrt{\left\langle\frac{1}{2}\left|E_{m}(H ; t)\right|^{2}\right\rangle}=\sqrt{I_{m, m}(H)}$.

The rms phases are zero for autocorrelations. Using the $n=0$ state as a reference, the rms phase of each state $m$ comes from the $(m, 0)$ cross correlation,

$\Delta \bar{\Phi}_{m, 0}(H)=\tan ^{-1} \frac{\operatorname{Im} I_{m, 0}(H)}{\operatorname{Re} I_{m, 0}(H)}=\tan ^{-1} \frac{\operatorname{Im}\left\langle\frac{1}{2} E_{m}(H ; t) E_{0}^{*}(H ; t)\right\rangle}{\operatorname{Re}\left\langle\frac{1}{2} E_{m}(H ; t) E_{0}^{*}(H ; t)\right\rangle}$.

The other cross correlations contain redundant rms phases, and they serve as a useful check.

An ensemble of limited measurements can often be used to gain valuable insights about astronomical sources. For example, the diameter of a star or the orbital parameters of a binary star can be extracted from a finite number of interferometric visibilities. For the POAM case, significant results can be obtained without referring to the electric fields at all. Consider the azimuthal Fourier series of the intensity

$I(\overrightarrow{\mathbf{H}})=\sum_{m=-\infty}^{\infty} \mathcal{I}_{m}(H) \mathrm{e}^{j m \epsilon}$,

where the

$I_{m}(H)=\frac{1}{2 \pi} \int_{0}^{2 \pi} \mathrm{d} \epsilon \mathrm{e}^{-j m \epsilon} I(\overrightarrow{\mathbf{H}})=\sum_{k=-\infty}^{\infty} I_{k, k-m}(H)$

are the components of the rancor spectrum. The POAM information contained in the rancors is not complete, but it may be sufficient for certain types of modeling (Sect. 9.2). As a matter of fact, if rancors are measured with sufficient spatial resolution, it is possible

2 I chose the word "rancor" because it is convenient and a synonym for polarization. Related expressions naturally follow, e.g., rancorimetry, rancorimeter, (un)rancored, and (de)rancorizer. 
to reconstruct an image using only this Fourier series. Note that each rancor is an infinite sum of correlations with the same rancor index $m$ (Appendix B).

Calculating $\hat{L}_{Z}^{\text {plane }}$ in terms of correlations is relatively straightforward. According to Appendix A, the azimuthal derivative of the aberration function is

$\frac{\partial \Phi(\overrightarrow{\mathbf{H}})}{\partial \epsilon}=\frac{1}{I(\overrightarrow{\mathbf{H}})} \operatorname{Re} \sum_{m=-\infty}^{\infty} \sum_{n=-\infty}^{\infty} n I_{m, n}(H) \mathrm{e}^{j(m-n) \epsilon}=\frac{1}{I(\overrightarrow{\mathbf{H}})}\left[\sum_{m=-\infty}^{\infty} m I_{m, m}(H)+2 \operatorname{Re} \sum_{m=-\infty}^{\infty} \sum_{n=m+1}^{\infty} n I_{m, n}(H) \mathrm{e}^{j(m-n) \epsilon}\right]$.

Substituting this equation into Eq. (4), I obtain the POAM expectation value

$\hat{L}_{Z}^{\text {plane }}=\sum_{m=-\infty}^{\infty} p_{m, m}^{\text {plane }} m \hbar$

where

$p_{m, m}^{\text {plane }}=\frac{I_{m, m}^{\text {plane }}}{I^{\text {plane }}}=\frac{2 \pi \int_{0}^{\infty} \mathrm{d} H H I_{m, m}(H)}{\int \mathrm{d}^{2} H I(\overrightarrow{\mathbf{H}})}=2 \pi \int_{0}^{\infty} \mathrm{d} H H p_{m, m}(H)$

is the probability of a single photon being in state $m, p_{m, m}(H)$ is the corresponding probability density, $I_{m, m}^{\text {plane }}$ is the integrated correlation $(m, m), I^{\text {plane }}$ is the intensity integrated over the plane,

$\sum_{m=-\infty}^{\infty} p_{m, m}^{\text {plane }}=\frac{I_{0}^{\text {plane }}}{I^{\text {plane }}}=2 \pi \int_{0}^{\infty} \mathrm{d} H H \frac{I_{0}(H)}{I^{\text {plane }}}=2 \pi \int_{0}^{\infty} \mathrm{d} H H \mathrm{p}_{0}(H)=1$,

$\mathcal{I}_{0}^{\text {plane }}$ is the integrated unrancored term, and $\mathrm{p}_{0}(H)$ is the unrancored probability density. The rancored probability densities contain phase infofrmation, but they do not contribute to $\hat{L}_{Z}^{\text {plane }}$ because the $\mathrm{e}^{j(m-n) \epsilon}$ are periodic in $\epsilon$.

\section{POAM propagation through a generic system}

In Sects. 2 and 3, I presented the POAM expansions of electric fields and intensities. Those expressions can be applied to any plane by simply changing the spatial coordinate $\overrightarrow{\mathbf{H}}$. In this section, I employ a mathematical systems approach using overlap integrals to describe how the POAM expansions of electric fields and intensities propagate through a generic system, from celestial sphere (input; $\overrightarrow{\mathbf{H}} \rightarrow \overrightarrow{\boldsymbol{\Omega}}$ ) to a specific plane (output; $\overrightarrow{\mathbf{H}} \rightarrow \overrightarrow{\mathbf{N}}$ ). These generic expansions can be applied to any optical system; I present important and very different examples in Sects. 5-9 to demonstrate their utility.

\subsection{Electric fields}

The expression for the propagation of electric fields is given by

$E(\overrightarrow{\mathbf{N}} ; \overrightarrow{\mathbf{a}}, t)=\int \mathrm{d}^{2} \Omega D(\overrightarrow{\mathbf{N}}, \overrightarrow{\boldsymbol{\Omega}} ; \overrightarrow{\mathbf{a}}) E(\overrightarrow{\boldsymbol{\Omega}} ; t)$,

where $\overrightarrow{\mathbf{N}}=\left(N_{x}, N_{y}\right)=(N \cos v, N \sin v)$ is the vector in the specific plane, $N_{x}$ and $N_{y}$ are the Cartestian coordinates in the specific plane, $N$ is the radial coordinate in the specific plane, $v$ is the azimuthal coordinate in the specific plane, $\overrightarrow{\boldsymbol{\Omega}}=(\alpha, \delta)=(\rho \cos \phi, \rho \sin \phi)$ is the vector on the celestial sphere, $\alpha$ and $\delta$ are the Cartestian coordinates on the celestial sphere, $\rho$ is the radial coordinate on the celestial sphere, $\phi$ is azimuthal coordinate on the celestial sphere, and $\overrightarrow{\mathbf{a}}$ is an optional list of parameters. This equation is an overlap integral, linking the input electric fields on the celestial sphere to the output electric fields in the specific plane via the system diffraction function (DF).

Equation (15) is valid for both spatially coherent and incoherent electric fields on the celestial sphere, but in this paper I deal only with the latter. The DF, which describes the propagation behavior through free space and instruments, could be a function of time in the presence of the atmosphere and thermomechanical instrument stresses. In the mathematical systems approach, the DF is also known as the electric-field impulse response.

When the electric fields and DF of Eq. (15) are expanded in azimuthal Fourier series, POAM propagation becomes clear. There are three different generic expansions, all displayed in Table 1. The formulae for the input sensitivities, output sensitivities, and input/output gains of the DF are located in Table 2. I derive these results in Appendix C.

The first electric-field expansion uses the input sensitivity of the DF. Each term indicates how the input states from the celestial sphere are modified by the instrument before they arrive at the specific plane. This expansion is of limited use because each component is not a specific-plane state, but I include it for the sake of completeness. Each term in the second expansion is a specific-plane state, written in terms of the output sensitivity and the total input electric field. This form is employed in situations where the exact form of the input POAM spectrum is not specified. The third expansion is a combination of the first two, using the input/output gains and the input states. The gains are direct indicators of how much source state $m$ is converted into specific-plane state $p$. This form may be used for a number of different purposes, including optical system analysis. 
Table 1. The POAM expansions of $E(\overrightarrow{\mathbf{N}} ; \overrightarrow{\mathbf{a}}, t)$ in terms of the POAM expansions of $D(\overrightarrow{\mathbf{N}}, \overrightarrow{\boldsymbol{\Omega}} ; \overrightarrow{\mathbf{a}})$ (Table 2) and $E(\overrightarrow{\boldsymbol{\Omega}} ; t)$.

\begin{tabular}{|c|c|}
\hline POAM expansion type & Expression \\
\hline Input & $\begin{array}{l}E(\overrightarrow{\mathbf{N}} ; \overrightarrow{\mathbf{a}}, t)=\sum_{m=-\infty}^{\infty} \hat{E}_{m}(\overrightarrow{\mathbf{N}} ; \overrightarrow{\mathbf{a}}, t) \\
\text { where } \hat{E}_{m}(\overrightarrow{\mathbf{N}} ; \overrightarrow{\mathbf{a}}, t)=2 \pi \int_{0}^{\infty} \mathrm{d} \rho \rho D^{-m}(\overrightarrow{\mathbf{N}}, \rho ; \overrightarrow{\mathbf{a}}) E_{m}(\rho ; t)\end{array}$ \\
\hline Output & $\begin{array}{l}E(\overrightarrow{\mathbf{N}} ; \overrightarrow{\mathbf{a}}, t)=\sum_{p=-\infty}^{\infty} E_{p}(N ; \overrightarrow{\mathbf{a}}, t) \mathrm{e}^{j p v} \\
\text { where } E_{p}(N ; \overrightarrow{\mathbf{a}}, t)=\int \mathrm{d}^{2} \Omega D_{p}(N, \overrightarrow{\boldsymbol{\Omega}} ; \overrightarrow{\mathbf{a}}) E(\overrightarrow{\boldsymbol{\Omega}} ; t)\end{array}$ \\
\hline Input/Output & $\begin{array}{l}E(\overrightarrow{\mathbf{N}} ; \overrightarrow{\mathbf{a}}, t)=\sum_{p=-\infty}^{\infty} E_{p}(N ; \overrightarrow{\mathbf{a}}, t) \mathrm{e}^{j p v} \\
\text { where } E_{p}(N ; \overrightarrow{\mathbf{a}}, t)=\sum_{m=-\infty}^{\infty} 2 \pi \int_{0}^{\infty} \mathrm{d} \rho \rho D_{p}^{-m}(N, \rho ; \overrightarrow{\mathbf{a}}) E_{m}(\rho ; t)\end{array}$ \\
\hline
\end{tabular}

Table 2. The POAM expansions of the $D(\overrightarrow{\mathbf{N}}, \overrightarrow{\boldsymbol{\Omega}} ; \overrightarrow{\mathbf{a}})$.

\begin{tabular}{l|l}
\hline \hline POAM expansion & Expression \\
\hline \hline Input sensitivity: & \\
Integral form (forward) & $D^{-m}(\overrightarrow{\mathbf{N}}, \rho ; \overrightarrow{\mathbf{a}})=\frac{1}{2 \pi} \int_{0}^{2 \pi} \mathrm{d} \phi \mathrm{e}^{j m \phi} D(\overrightarrow{\mathbf{N}}, \overrightarrow{\boldsymbol{\Omega}} ; \overrightarrow{\mathbf{a}})$ \\
Sum form (reverse) & $D(\overrightarrow{\mathbf{N}}, \overrightarrow{\boldsymbol{\Omega}} ; \overrightarrow{\mathbf{a}})=\sum_{m=-\infty}^{\infty} D^{-m}(\overrightarrow{\mathbf{N}}, \rho ; \overrightarrow{\mathbf{a}}) \mathrm{e}^{-j m \phi}$ \\
\hline \hline Output sensitivity: & \\
Integral form (forward) & $D_{p}(N, \overrightarrow{\mathbf{\Omega}} ; \overrightarrow{\mathbf{a}})=\frac{1}{2 \pi} \int_{0}^{2 \pi} \mathrm{d} v \mathrm{e}^{-j p v} D(\overrightarrow{\mathbf{N}}, \overrightarrow{\boldsymbol{\Omega}} ; \overrightarrow{\mathbf{a}})$ \\
Sum form (reverse) & $D(\overrightarrow{\mathbf{N}}, \overrightarrow{\boldsymbol{\Omega}} ; \overrightarrow{\mathbf{a}})=\sum_{p=-\infty}^{\infty} D_{p}(N, \overrightarrow{\boldsymbol{\Omega}} ; \overrightarrow{\mathbf{a}}) \mathrm{e}^{j p v}$ \\
\hline \hline Input/Output gain: & \\
Integral form (forward) & $D_{p}^{-m}(N, \rho ; \overrightarrow{\mathbf{a}})=\frac{1}{2 \pi} \int_{0}^{2 \pi} \mathrm{d} \phi \mathrm{e}^{j m \phi} \frac{1}{2 \pi} \int_{0}^{2 \pi} \mathrm{d} v \mathrm{e}^{-j p v} D(\overrightarrow{\mathbf{N}}, \overrightarrow{\boldsymbol{\Omega}} ; \overrightarrow{\mathbf{a}})$ \\
Sum form (reverse) & $D(\overrightarrow{\mathbf{N}}, \overrightarrow{\boldsymbol{\Omega}} ; \overrightarrow{\mathbf{a}})=\sum_{p=-\infty}^{\infty} \sum_{m=-\infty}^{\infty} D_{p}^{-m}(N, \rho ; \overrightarrow{\mathbf{a}}) \mathrm{e}^{-j m \phi} \mathrm{e}^{j p v}$ \\
\hline
\end{tabular}

\subsection{Intensities}

For spatially incoherent electric fields on the celestial sphere, the expression for the propagation of intensities has the same overlapintegral form as Eq. (15),

$I(\overrightarrow{\mathbf{N}} ; \overrightarrow{\mathbf{a}})=\left\langle\frac{1}{2}|E(\overrightarrow{\mathbf{N}} ; \overrightarrow{\mathbf{a}}, t)|^{2}\right\rangle=\int \mathrm{d}^{2} \Omega P(\overrightarrow{\mathbf{N}}, \overrightarrow{\mathbf{\Omega}} ; \overrightarrow{\mathbf{a}}) I(\overrightarrow{\mathbf{\Omega}})=\int \mathrm{d}^{2} \Omega|D(\overrightarrow{\mathbf{N}}, \overrightarrow{\mathbf{\Omega}} ; \overrightarrow{\mathbf{a}})|^{2}\left\langle\frac{1}{2}|E(\overrightarrow{\mathbf{\Omega}} ; t)|^{2}\right\rangle$

I derive this equation in Appendix D. The intensity impulse response of the mathematical systems approach is more commonly known as the point-spread function (PSF) in astronomy.

When the input electric fields and DF of Eq. (15) are expanded in azimuthal Fourier series and plugged into Eq. (16), POAM propagation becomes clear. There are eight different generic expansions involving both correlations and rancors, all displayed in Table 3. The formulae for the input sensitivities, output sensitivities, and input/output gains of the PSF are located in Table 4. I derive these results in Appendix C.

The first two expansions use the correlated and rancored input sensitivities of the PSF. Each term shows how the input correlations or rancors are modified by the instrument before they arrive at the specific plane. These expansions are of limited use because each component is not a specific-plane correlation or rancor, but I include them for the sake of completeness. Each term in the next two expansions is either a correlation or rancor, written in terms of the total input intensity. These forms are employed in situations where the exact forms of the input correlation or rancor spectra are not specified. The last four expansions are combinations of the first two, using the input/output gains and the input correlations and rancors. The gains are a direct indication of how much input correlation $(m, n)$ (or rancor $m$ ) is converted into specific-plane correlation $(p, q)$ (or rancor $p$ ). These forms may be used for a number of different purposes, including optical system analysis.

\section{POAM propagation through free space}

Spatially incoherent electric fields on the celestial sphere $(\overrightarrow{\mathbf{H}} \rightarrow \overrightarrow{\boldsymbol{\Omega}})$ travel through free space to the observation plane $(\overrightarrow{\mathbf{H}} \rightarrow \overrightarrow{\mathbf{R}})$, which contains the entrance pupil of the instrument. In this section, I form the DF, PSF, and the POAM expansions for this simple system and prove that 1) each state on the celestial sphere gives rise to only the same state in the observation plane; 2) only the unrancored correlations in the observation are non-zero; and 3) the integrated probabilities and POAM expectation value are conserved, which means that no external torque is applied to the wavefronts.

\subsection{Classical forms}

The DF for propagation through free space is

$D(\overrightarrow{\mathbf{N}}, \overrightarrow{\boldsymbol{\Omega}} ; \overrightarrow{\mathbf{a}}) \rightarrow D(\overrightarrow{\mathbf{R}}, \overrightarrow{\boldsymbol{\Omega}})=\mathrm{e}^{j 2 \pi k \overrightarrow{\mathbf{R}} \cdot \hat{\mathbf{s}}(\overrightarrow{\boldsymbol{\Omega}})}=\mathrm{e}^{j 2 \pi k \overrightarrow{\mathbf{R}} \cdot \hat{\mathbf{x}}_{x y}(\overrightarrow{\boldsymbol{\Omega}})} \approx \mathrm{e}^{j 2 \pi \kappa R \rho \cos (\psi-\phi)}$ 
Table 3. The POAM expansions of $I(\overrightarrow{\mathbf{N}} ; \overrightarrow{\mathbf{a}})$, for a spatially incoherent source, in terms of the POAM expansions of $P(\overrightarrow{\mathbf{N}}, \overrightarrow{\boldsymbol{\Omega}} ; \overrightarrow{\mathbf{a}})($ Table 4$)$ and $I(\overrightarrow{\mathbf{\Omega}})$.

\begin{tabular}{|c|c|}
\hline POAM expansion type & Expression \\
\hline $\begin{array}{l}\text { Input } \\
\text { (correlated) }\end{array}$ & $\begin{array}{l}I(\overrightarrow{\mathbf{N}} ; \overrightarrow{\mathbf{a}})=\sum_{m=-\infty}^{\infty} \sum_{n=-\infty}^{\infty} \hat{I}_{m, n}(\overrightarrow{\mathbf{N}} ; \overrightarrow{\mathbf{a}}) \\
\text { where } \hat{I}_{m, n}(\overrightarrow{\mathbf{N}} ; \overrightarrow{\mathbf{a}})=2 \pi \int_{0}^{\infty} \mathrm{d} \rho \rho \mathcal{P}^{-m+n}(\overrightarrow{\mathbf{N}}, \rho ; \overrightarrow{\mathbf{a}}) I_{m, n}(\rho)\end{array}$ \\
\hline $\begin{array}{l}\text { Input } \\
\text { (rancored) }\end{array}$ & $\begin{array}{l}I(\overrightarrow{\mathbf{N}} ; \overrightarrow{\mathbf{a}})=\sum_{m=-\infty}^{\infty} \hat{\mathcal{I}}_{m}(\overrightarrow{\mathbf{N}} ; \overrightarrow{\mathbf{a}}) \\
\text { where } \hat{\mathcal{I}}_{m}(\overrightarrow{\mathbf{N}} ; \overrightarrow{\mathbf{a}})=2 \pi \int_{0}^{\infty} \mathrm{d} \rho \rho \mathcal{P}^{-m}(\overrightarrow{\mathbf{N}}, \rho ; \overrightarrow{\mathbf{a}}) \mathcal{I}_{m}(\rho)\end{array}$ \\
\hline $\begin{array}{l}\text { Output } \\
\text { (correlated/unexpanded) }\end{array}$ & $\begin{array}{l}I(\overrightarrow{\mathbf{N}} ; \overrightarrow{\mathbf{a}})=\sum_{p=-\infty}^{\infty} \sum_{q=-\infty}^{\infty} I_{p, q}(N ; \overrightarrow{\mathbf{a}}) \mathrm{e}^{j(p-q) v} \\
\text { where } I_{p, q}(N ; \overrightarrow{\mathbf{a}})=\int \mathrm{d}^{2} \Omega P_{p, q}(N, \overrightarrow{\boldsymbol{\Omega}} ; \overrightarrow{\mathbf{a}}) I(\overrightarrow{\boldsymbol{\Omega}})\end{array}$ \\
\hline $\begin{array}{l}\text { Output } \\
\text { (rancored/unexpanded) }\end{array}$ & $\begin{array}{l}I(\overrightarrow{\mathbf{N}} ; \overrightarrow{\mathbf{a}})=\sum_{p=-\infty}^{\infty} I_{p}(N ; \overrightarrow{\mathbf{a}}) \mathrm{e}^{j p v} \\
\text { where } \mathcal{I}_{p}(N ; \overrightarrow{\mathbf{a}})=\int \mathrm{d}^{2} \Omega \mathcal{P}_{p}(N, \overrightarrow{\boldsymbol{\Omega}} ; \overrightarrow{\mathbf{a}}) I(\overrightarrow{\boldsymbol{\Omega}})\end{array}$ \\
\hline $\begin{array}{l}\text { Input/Output } \\
\text { (correlated/correlated) }\end{array}$ & $\begin{array}{l}I(\overrightarrow{\mathbf{N}} ; \overrightarrow{\mathbf{a}})=\sum_{p=-\infty}^{\infty} \sum_{q=-\infty}^{\infty} I_{p, q}(N ; \overrightarrow{\mathbf{a}}) \mathrm{e}^{j(p-q) v} \\
\text { where } I_{p, q}(N ; \overrightarrow{\mathbf{a}})=\sum_{m=-\infty}^{\infty} \sum_{n=-\infty}^{\infty} 2 \pi \int_{0}^{\infty} \mathrm{d} \rho \rho \mathcal{P}_{p, q}^{-m+n}(N, \rho ; \overrightarrow{\mathbf{a}}) I_{m, n}(\rho)\end{array}$ \\
\hline $\begin{array}{l}\text { Input/Output } \\
\text { (correlated/rancored) }\end{array}$ & $\begin{array}{l}I(\overrightarrow{\mathbf{N}} ; \overrightarrow{\mathbf{a}})=\sum_{p=-\infty}^{\infty} \sum_{q=-\infty}^{\infty} I_{p, q}(N ; \overrightarrow{\mathbf{a}}) \mathrm{e}^{j(p-q) v} \\
\text { where } I_{p, q}(N ; \overrightarrow{\mathbf{a}})=\sum_{m=-\infty}^{\infty} 2 \pi \int_{0}^{\infty} \mathrm{d} \rho \rho \mathcal{P}_{p, q}^{-m}(N, \rho ; \overrightarrow{\mathbf{a}}) I_{m}(\rho)\end{array}$ \\
\hline $\begin{array}{l}\text { Input/Output } \\
\text { (rancored/correlated) }\end{array}$ & $\begin{array}{l}I(\overrightarrow{\mathbf{N}} ; \overrightarrow{\mathbf{a}})=\sum_{p=-\infty}^{\infty} I_{p}(N ; \overrightarrow{\mathbf{a}}) \mathrm{e}^{j p v} \\
\text { where } I_{p}(N ; \overrightarrow{\mathbf{a}})=\sum_{m=-\infty}^{\infty} \sum_{n=-\infty}^{\infty} 2 \pi \int_{0}^{\infty} \mathrm{d} \rho \rho \mathcal{P}_{p}^{-m+n}(N, \rho ; \overrightarrow{\mathbf{a}}) I_{m, n}(\rho)\end{array}$ \\
\hline $\begin{array}{l}\text { Input/Output } \\
\text { (rancored/rancored) }\end{array}$ & $\begin{array}{l}I(\overrightarrow{\mathbf{N}} ; \overrightarrow{\mathbf{a}})=\sum_{p=-\infty}^{\infty} \mathcal{I}_{p}(N ; \overrightarrow{\mathbf{a}}) \mathrm{e}^{j p v} \\
\text { where } \mathcal{I}_{p}(N ; \overrightarrow{\mathbf{a}})=\sum_{m=-\infty}^{\infty} 2 \pi \int_{0}^{\infty} \mathrm{d} \rho \rho \mathcal{P}_{p}^{-m}(N, \rho ; \overrightarrow{\mathbf{a}}) \mathcal{I}_{m}(\rho)\end{array}$ \\
\hline
\end{tabular}

Table 4. The POAM expansions of $P(\overrightarrow{\mathbf{N}}, \overrightarrow{\mathbf{\Omega}} ; \overrightarrow{\mathbf{a}})$.

\begin{tabular}{|c|c|}
\hline POAM expansion & Expression \\
\hline $\begin{array}{l}\text { Input sensitivity (separate): } \\
\text {. }\end{array}$ & \\
\hline Integral form (forward) & $P^{-m,-n}(\overrightarrow{\mathbf{N}}, \rho ; \overrightarrow{\mathbf{a}})=D^{-m}(\overrightarrow{\mathbf{N}}, \rho ; \overrightarrow{\mathbf{a}}) D^{-n, *}(\overrightarrow{\mathbf{N}}, \rho ; \overrightarrow{\mathbf{a}})$ \\
\hline Sum form (reverse) & $P(\overrightarrow{\mathbf{N}}, \overrightarrow{\boldsymbol{\Omega}} ; \overrightarrow{\mathbf{a}})=\sum_{m=-\infty}^{\infty} \sum_{n=-\infty}^{\infty} P^{-m,-n}(\overrightarrow{\mathbf{N}}, \rho ; \overrightarrow{\mathbf{a}}) \mathrm{e}^{-j(m-n) \phi}$ \\
\hline Output sensitivity (separate): & \\
\hline Integral form (forward) & $P_{p, q}(N, \overrightarrow{\boldsymbol{\Omega}} ; \overrightarrow{\mathbf{a}})=D_{p}(N, \overrightarrow{\boldsymbol{\Omega}} ; \overrightarrow{\mathbf{a}}) D_{q}^{*}(N, \overrightarrow{\boldsymbol{\Omega}} ; \overrightarrow{\mathbf{a}})$ \\
\hline Sum form (reverse) & $P(\overrightarrow{\mathbf{N}}, \overrightarrow{\boldsymbol{\Omega}} ; \overrightarrow{\mathbf{a}})=\sum_{p=-\infty}^{\infty} \sum_{q=-\infty}^{\infty} P_{p, q}(N, \overrightarrow{\boldsymbol{\Omega}} ; \overrightarrow{\mathbf{a}}) \mathrm{e}^{j(p-q) v}$ \\
\hline Input/Output gain (separate): & \\
\hline Integral form (forward) & $P_{p, q}^{-m,-n}(N, \rho ; \overrightarrow{\mathbf{a}})=D_{p}^{-m}(N, \rho ; \overrightarrow{\mathbf{a}}) D_{q}^{-n, *}(N, \rho ; \overrightarrow{\mathbf{a}})$ \\
\hline Sum form (reverse) & $P(\overrightarrow{\mathbf{N}}, \overrightarrow{\boldsymbol{\Omega}} ; \overrightarrow{\mathbf{a}})=\sum_{p=-\infty}^{\infty} \sum_{q=-\infty}^{\infty} \sum_{m=-\infty}^{\infty} \sum_{n=-\infty}^{\infty} P_{p, q}^{-m,-n}(N, \rho ; \overrightarrow{\mathbf{a}}) \mathrm{e}^{-j(m-n) \phi} \mathrm{e}^{j(p-q) v}$ \\
\hline Input sensitivity (combined): & \\
\hline Integral form (forward) & $\mathcal{P}^{-m}(\overrightarrow{\mathbf{N}}, \rho ; \overrightarrow{\mathbf{a}})=\frac{1}{2 \pi} \int_{0}^{2 \pi} \mathrm{d} \phi \mathrm{e}^{j m \phi} P(\overrightarrow{\mathbf{N}}, \overrightarrow{\boldsymbol{\Omega}} ; \overrightarrow{\mathbf{a}})=\sum_{k=-\infty}^{\infty} P^{-k,-k+m}(\overrightarrow{\mathbf{N}}, \rho ; \overrightarrow{\mathbf{a}})$ \\
\hline Sum form (reverse) & $P(\overrightarrow{\mathbf{N}}, \overrightarrow{\boldsymbol{\Omega}} ; \overrightarrow{\mathbf{a}})=\sum_{m=-\infty}^{\infty} \mathcal{P}^{-m}(\overrightarrow{\mathbf{N}}, \rho ; \overrightarrow{\mathbf{a}}) \mathrm{e}^{-j m \phi}$ \\
\hline Output sensitivity (combined): & \\
\hline Integral form (forward) & $\mathcal{P}_{p}(N, \overrightarrow{\boldsymbol{\Omega}} ; \overrightarrow{\mathbf{a}})=\frac{1}{2 \pi} \int_{0}^{2 \pi} \mathrm{d} v \mathrm{e}^{-j p v} P(\overrightarrow{\mathbf{N}}, \overrightarrow{\boldsymbol{\Omega}} ; \overrightarrow{\mathbf{a}})=\sum_{l=-\infty}^{\infty} P_{l, l-p}(N, \overrightarrow{\boldsymbol{\Omega}} ; \overrightarrow{\mathbf{a}})$ \\
\hline Sum form (reverse) & $P(\overrightarrow{\mathbf{N}}, \overrightarrow{\boldsymbol{\Omega}} ; \overrightarrow{\mathbf{a}})=\sum_{p=-\infty}^{\infty} \mathcal{P}_{p}(N, \overrightarrow{\boldsymbol{\Omega}} ; \overrightarrow{\mathbf{a}}) \mathrm{e}^{j p v}$ \\
\hline$\overline{\text { Input/Output gain (combined \#1): }}$ & \\
\hline Integral form (forward) & $\mathcal{P}_{p}^{-m}(N, \rho ; \overrightarrow{\mathbf{a}})=\frac{1}{2 \pi} \int_{0}^{2 \pi} \mathrm{d} \phi \mathrm{e}^{j m \phi} \frac{1}{2 \pi} \int_{0}^{2 \pi} \mathrm{d} v \mathrm{e}^{-j p v} P(\overrightarrow{\mathbf{N}}, \overrightarrow{\mathbf{\Omega}} ; \overrightarrow{\mathbf{a}})=\sum_{k=-\infty}^{\infty} \sum_{l=-\infty}^{\infty} P_{l, l-p}^{-k,-k+m}(N, \rho ; \overrightarrow{\mathbf{a}})$ \\
\hline Sum form (reverse) & $P(\overrightarrow{\mathbf{N}}, \overrightarrow{\boldsymbol{\Omega}} ; \overrightarrow{\mathbf{a}})=\sum_{p=-\infty}^{\infty} \sum_{m=-\infty}^{\infty} \mathcal{P}_{p}^{-m}(N, \rho ; \overrightarrow{\mathbf{a}}) \mathrm{e}^{-j m \phi} \mathrm{e}^{j p v}$ \\
\hline Input/Output gain (combined \#2): & \\
\hline Integral form (forward) & $\mathcal{P}_{p, q}^{-m}(N, \rho ; \overrightarrow{\mathbf{a}})=\frac{1}{2 \pi} \int_{0}^{2 \pi} \mathrm{d} \phi \mathrm{e}^{j m \phi} P_{p, q}(N, \overrightarrow{\mathbf{\Omega}} ; \overrightarrow{\mathbf{a}})=\sum_{k=-\infty}^{\infty} P_{p, q}^{-k,-k+m}(N, \rho ; \overrightarrow{\mathbf{a}})$ \\
\hline Sum form (reverse) & $P(\overrightarrow{\mathbf{N}}, \overrightarrow{\boldsymbol{\Omega}} ; \overrightarrow{\mathbf{a}})=\sum_{p=-\infty}^{\infty} \sum_{q=-\infty}^{\infty} \sum_{m=-\infty}^{\infty} \mathcal{P}_{p, q}^{-m}(N, \rho ; \overrightarrow{\mathbf{a}}) \mathrm{e}^{-j m \phi} \mathrm{e}^{j(p-q) v}$ \\
\hline Input/Output gain (combined \#3): & \\
\hline Integral form (forward) & $\mathcal{P}_{p}^{-m,-n}(N, \rho)=\frac{1}{2 \pi} \int_{0}^{2 \pi} \mathrm{d} v \mathrm{e}^{-j p v} P^{-m,-n}(\overrightarrow{\mathbf{N}}, \rho ; \overrightarrow{\mathbf{a}})=\sum_{l=-\infty}^{\infty} P_{l, l-p}^{-m,-n}(N, \rho ; \overrightarrow{\mathbf{a}})$ \\
\hline Sum form (reverse) & $P(\overrightarrow{\mathbf{N}}, \overrightarrow{\boldsymbol{\Omega}} ; \overrightarrow{\mathbf{a}})=\sum_{p=-\infty}^{\infty} \sum_{m=-\infty}^{\infty} \sum_{n=-\infty}^{\infty} \mathcal{P}_{p}^{-m,-n}(N, \rho ; \overrightarrow{\mathbf{a}}) \mathrm{e}^{-j(m-n) \phi} \mathrm{e}^{j p v}$ \\
\hline
\end{tabular}

where $\overrightarrow{\mathbf{R}}=(X, Y)=(R \cos \psi, R \sin \psi)$ is the vector in the observation plane, $X$ and $Y$ are the Cartesian coordinates, $R$ is the radial coordinate, $\psi$ is azimuthal coordinate, $\kappa=1 / \lambda$ is the wavenumber,

$\hat{\mathbf{S}}(\overrightarrow{\mathbf{\Omega}})=\sin \rho \cos \phi \hat{\mathbf{X}}+\sin \rho \sin \phi \hat{\mathbf{Y}}+\cos \rho \hat{\mathbf{Z}}=\overrightarrow{\mathbf{s}}_{x y}(\overrightarrow{\mathbf{\Omega}})+\cos \rho \hat{\mathbf{Z}} \approx \rho \cos \phi \hat{\mathbf{X}}+\rho \sin \phi \hat{\mathbf{Y}}+1 \hat{\mathbf{Z}}$ 
is the unit vector pointing toward the celestial sphere, and $\hat{\mathbf{X}}, \hat{\mathbf{Y}}$, and $\hat{\mathbf{Z}}$ are the unit vectors originating from the observation plane. Only $\hat{\mathbf{s}}_{x y}(\overrightarrow{\boldsymbol{\Omega}})$ is required, since $\overrightarrow{\mathbf{R}}$ and $\hat{\mathbf{Z}}$ are perpendicular. The approximation in Eq. (17b) arises because the distance between the celestial sphere and the observation plane is extremely large. It leads to the approximation in Eq. (17a), whose exponential kernel is the Fraunhofer propagator. The electric field in the observation plane then becomes

$E(\overrightarrow{\mathbf{N}} ; \overrightarrow{\mathbf{a}}, t) \rightarrow E(\overrightarrow{\mathbf{R}} ; t)=\int \mathrm{d}^{2} \Omega \mathrm{e}^{j 2 \pi \kappa R \rho \cos (\psi-\phi)} E(\overrightarrow{\boldsymbol{\Omega}} ; t)$.

Also, this DF leads to a PSF of unity, which means that the intensity becomes

$I(\overrightarrow{\mathbf{N}} ; \overrightarrow{\mathbf{a}}) \rightarrow I(\overrightarrow{\mathbf{R}})=\int \mathrm{d}^{2} \Omega I(\overrightarrow{\mathbf{\Omega}})=I^{\mathrm{cs}}=$ constant

A constant intensity over the entire observation plane suggests that the source emits an infinite amount of energy, which is impossible. In reality, the intensity is constant over a very large sphere centered on the source. Locally, each point on the sphere may be well approximated by an observation plane that is larger than the entrance pupil of any practical astronomical telescope, which means that the intensity integral over the entrance pupil

$I^{\mathrm{ent}}=\int_{\mathrm{ent}} \mathrm{d}^{2} R I(\overrightarrow{\mathbf{R}})=A_{\mathrm{ent}} I^{\mathrm{cs}}=$ constant

will be finite, where $A_{\text {ent }}$ is the area of the entrance pupil.

\subsection{POAM expansions}

Each state in the observation plane has the form

$E_{m}(R ; t)=2 \pi \int_{0}^{\infty} \mathrm{d} \rho \rho D_{m}^{-m}(R, \rho) E_{m}(\rho ; t)=j^{m} 2 \pi \int_{0}^{\infty} \mathrm{d} \rho \rho J_{m}(2 \pi \kappa R \rho) E_{m}(\rho ; t)=j^{m} H_{m}\left[E_{m}(\rho ; t) ; R\right]$,

where $H_{m}\left[E_{m}(\rho ; t) ; R\right]$ is the $m$ th-order Hankel transform (Bracewell 1986), and $J_{m}(\cdot)$ is the $m$ th-order Bessel function of the first kind (Appendix E). This equation shows that each state on the celestial sphere gives rise only to the same state in the observation plane, which means that propagation through free space does not apply external torque. A small uniform $m \neq 0$ disk at the FOV center produces decaying rings with large radii in the observation plane. As the disk radius increases, the ring radii decrease.

The correlations in the observation plane are

$I_{m, n}(R)=2 \pi \int_{0}^{\infty} \mathrm{d} \rho \rho P_{m, n}^{-m,-n}(R, \rho) \mathcal{I}_{m-n}(\rho)=j^{m-n} 2 \pi \int_{0}^{\infty} \mathrm{d} \rho \rho J_{m}(2 \pi \kappa R \rho) J_{n}(2 \pi \kappa R \rho) \mathcal{I}_{m-n}(\rho)$.

Only the unrancored correlations in the observation plane are non-zero, which means that

$I(\overrightarrow{\mathbf{R}})=\mathcal{I}_{0}(R)=\sum_{m=-\infty}^{\infty} I_{m, m}(R)=\sum_{m=-\infty}^{\infty} 2 \pi \int_{0}^{\infty} \mathrm{d} \rho \rho I_{m, m}(\rho)=\sum_{m=-\infty}^{\infty} I_{m, m}^{\mathrm{cs}}$

where the second last equality comes from Appendix E, and the last equality comes from Eq. (14b). Not only is the sum constant (Eq. (18a)), but each term is constant as well. Using Eq. (14b) again, I find that

$p_{m, m}^{\mathrm{ent}}=\frac{2 \pi \int_{0}^{R_{\mathrm{ent}}} \mathrm{d} R R I_{m, m}(R)}{I^{\mathrm{ent}}}=\frac{A^{\mathrm{ent}} I_{m, m}^{\mathrm{cs}}}{A^{\mathrm{ent}} I^{\mathrm{cs}}}=p_{m, m}^{\mathrm{cs}}$,

where $R_{\text {ent }}$ is the radius of the entrance pupil. The integrated probability in the entrance pupil is identical to the integrated probability on the celestial sphere. Therefore, the POAM expectation value is conserved $\left(\hat{L}_{Z}^{\text {ent }}=\hat{L}_{Z}^{\text {cs }}\right)$ and no external torque is applied to the wavefronts, as shown previously.

\section{POAM propagation through a single telescope}

Electric fields in the observation plane $(\overrightarrow{\mathbf{H}} \rightarrow \overrightarrow{\mathbf{R}})$, which originate from a spatially incoherent source on the celestial sphere $(\overrightarrow{\mathbf{H}} \rightarrow$ $\overrightarrow{\boldsymbol{\Omega}})$ and propagate through free space, must be sent to an instrument for analysis $(\overrightarrow{\mathbf{H}} \rightarrow \overrightarrow{\mathbf{r}})$. In this section, I employ a telescope for this task (Fig. 1). The telescope has an exit pupil, which represents the image of the entrance pupil plus aberrations. The exit pupil is included so that POAM devices (e.g., mask, hologram, sorter, etc.) or non-POAM devices (e.g., deformable mirror) may be introduced into the optical system. I form the DF, PSF, and the POAM expansions for an unaberrated telescope and prove that 1) the input sensitivities, output sensitivities, and input/output gains of the DF can be expressed in terms the same principal DF POAM functions; 2) the sensitivities, output sensitivities, and input/output gains of the PSF can be expressed in terms of the same principal PSF functions; 3 ) the integrated probabilities and POAM expectation values are conserved, which means that no external torque is applied to the wavefronts. 
telescope

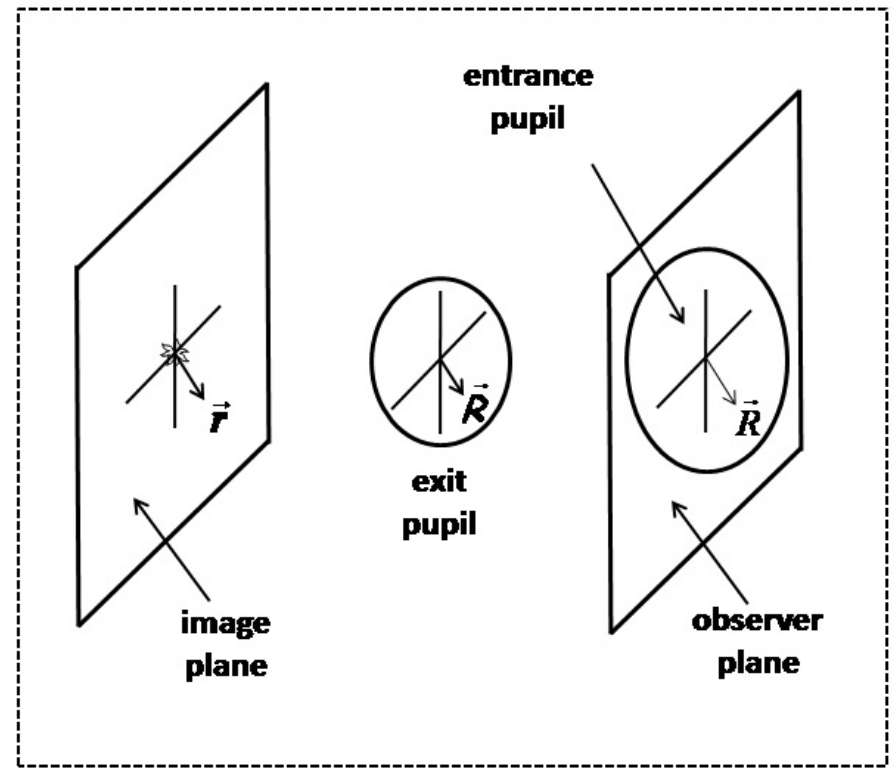

free

space

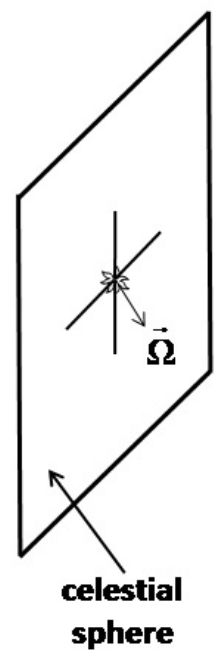

Fig. 1. The schematic diagram of propagation from a source on the celestial sphere through free space and telescope to the image plane.

\subsection{Classical forms}

When the integral over $\overrightarrow{\boldsymbol{\Omega}}$ in Eq. (17c) is removed, the result is the electric field in the observation plane due to a point source (impulse) on the celestial sphere, or

$E(\overrightarrow{\mathbf{R}}, \overrightarrow{\boldsymbol{\Omega}} ; t)=\mathrm{e}^{j 2 \pi \kappa \mathbf{\mathbf { R }} \cdot \overrightarrow{\mathbf{s}}_{x y}(\overrightarrow{\boldsymbol{\Omega}})} E(\overrightarrow{\mathbf{\Omega}} ; t)$

The electric field in the exit-pupil plane is a modified version of Eq. (22a)

$E(\overrightarrow{\mathcal{R}}, \overrightarrow{\boldsymbol{\Omega}} ; t)=\mathrm{e}^{j 2 \pi \kappa M \overrightarrow{\mathcal{R}} \cdot \overrightarrow{\mathbf{s}}_{x y}(\overrightarrow{\boldsymbol{\Omega}})} \mathcal{D}(\overrightarrow{\mathcal{R}}, \overrightarrow{\boldsymbol{\Omega}}) E(\overrightarrow{\mathbf{\Omega}} ; t)$,

where $\overrightarrow{\mathcal{R}}=\overrightarrow{\mathbf{R}} / M=(\mathcal{X}, \mathcal{Y})=(\mathcal{R} \cos \chi, \mathcal{R} \sin \chi)$ is the vector in the the exit-pupil plane, $\mathcal{X}$ and $\mathcal{Y}$ are the Cartesian coordinates, $\mathcal{R}$ is the radial coordinate, $\chi$ is the azimuthal coordinate, $\mathcal{D}(\overrightarrow{\mathcal{R}}, \overrightarrow{\boldsymbol{\Omega}})$ is an amplitude/phase aberration function of the instrument front-end projected into the exit-pupil plane, and $M$ is the amount of beam compression between the entrance- and exit-pupil planes. The electric field in the image plane due to a point source on the celestial sphere is

$E(\overrightarrow{\mathbf{r}}, \overrightarrow{\boldsymbol{\Omega}} ; t)=\frac{1}{A_{\mathrm{ep}}} \int \mathrm{d}^{2} \mathcal{R} \mathrm{e}^{-j 2 \pi \kappa \frac{\overrightarrow{\mathbf{r}}}{f} \cdot \overrightarrow{\mathcal{R}}} E(\overrightarrow{\mathcal{R}}, \overrightarrow{\boldsymbol{\Omega}} ; t)=D(\overrightarrow{\mathbf{r}}, \overrightarrow{\boldsymbol{\Omega}}) E(\overrightarrow{\boldsymbol{\Omega}} ; t)$,

where

$D(\overrightarrow{\mathbf{r}}, \overrightarrow{\boldsymbol{\Omega}})=\frac{1}{A_{\mathrm{ep}}} \int \mathrm{d}^{2} \mathcal{R} \mathrm{e}^{-j 2 \pi \kappa M\left[\frac{\overrightarrow{\mathbf{r}}}{\Theta}-\overrightarrow{\mathbf{s}}_{x y}(\overrightarrow{\boldsymbol{\Omega}})\right] \cdot \overrightarrow{\mathcal{R}}} \mathcal{D}(\overrightarrow{\mathcal{R}}, \overrightarrow{\boldsymbol{\Omega}})$

is the telescope DF, $A_{\mathrm{ep}}=\pi R_{\mathrm{ep}}^{2}$ is the area of the exit pupil, $R_{\mathrm{ep}}$ is the radius of the exit pupil, $\Theta=M f$ is the plate scale (units are length per angle, e.g. $\left.\mu \mathrm{m} \operatorname{arcsec}^{-1}\right), f$ is the focal length between the exit-pupil plane and the image plane, $\overrightarrow{\mathbf{r}}=(x, y)=$ $(r \cos \xi, r \sin \xi)$ is the vector, $x$ and $y$ are the Cartesian coordinates, $r$ is the radial coordinate, and $\xi$ is the azimuthal coordinate. The overlap integrals,

$E(\overrightarrow{\mathbf{r}} ; t)=\int \mathrm{d}^{2} \Omega D(\overrightarrow{\mathbf{r}}, \overrightarrow{\mathbf{\Omega}}) E(\overrightarrow{\mathbf{\Omega}} ; t)$

and

$I(\overrightarrow{\mathbf{r}})=\int \mathrm{d}^{2} \Omega P(\overrightarrow{\mathbf{r}}, \overrightarrow{\mathbf{\Omega}}) I(\overrightarrow{\mathbf{\Omega}})=\int \mathrm{d}^{2} \Omega|D(\overrightarrow{\mathbf{r}}, \overrightarrow{\boldsymbol{\Omega}})|^{2}\left\langle\frac{1}{2}|E(\overrightarrow{\mathbf{r}} ; t)|^{2}\right\rangle$,

are expressed in terms of the DF and PSF and have the same forms as in Sect. $4(\overrightarrow{\mathbf{N}} \rightarrow \overrightarrow{\mathbf{r}}$ and $\overrightarrow{\mathbf{a}} \rightarrow \overrightarrow{\mathbf{0}})$.

Consider an unaberrated telescope with $\mathcal{D}(\overrightarrow{\mathcal{R}}, \overrightarrow{\boldsymbol{\Omega}}) \rightarrow \mathcal{D}_{0}=$ real constant (the phase is arbitrary, so I choose zero). The DF then becomes

$D(\overrightarrow{\mathbf{r}}, \overrightarrow{\mathbf{\Omega}}) \rightarrow \mathbf{D}(\overrightarrow{\mathbf{r}}, \overrightarrow{\mathbf{\Omega}})=\mathcal{D}_{0} \mathcal{J}_{1}\left[2 \pi \kappa R_{\mathrm{tel}} \Gamma(\overrightarrow{\mathbf{r}}, \overrightarrow{\mathbf{\Omega}})\right]$ 
where

$\Gamma(\overrightarrow{\mathbf{r}}, \overrightarrow{\boldsymbol{\Omega}})=\left|\frac{\overrightarrow{\mathbf{r}}}{\Theta}-\overrightarrow{\mathbf{s}}_{x y}(\overrightarrow{\boldsymbol{\Omega}})\right|$

is the length of the special coordinate relating a position on the celestial sphere to a position in the image plane, $R_{\mathrm{tel}}=M R_{\mathrm{ep}}$ is the radius of the telescope entrance pupil, and $\mathcal{J}_{1}(x)=2 J_{1}(x) / x$. The PSF of an unaberrated telescope is simply the Airy function, or

$P(\overrightarrow{\mathbf{r}}, \overrightarrow{\mathbf{\Omega}}) \rightarrow \mathbf{P}(\overrightarrow{\mathbf{r}}, \overrightarrow{\mathbf{\Omega}})=|\mathbf{D}(\overrightarrow{\mathbf{r}}, \overrightarrow{\mathbf{\Omega}})|^{2}=\mathcal{D}_{0}^{2} \mathcal{J}_{1}^{2}\left[2 \pi \kappa R_{\mathrm{tel}} \Gamma(\overrightarrow{\mathbf{r}}, \overrightarrow{\mathbf{\Omega}})\right]=\mathcal{P}_{0} \mathcal{A}\left[2 \pi \kappa R_{\mathrm{tel}} \Gamma(\overrightarrow{\mathbf{r}}, \overrightarrow{\mathbf{\Omega}})\right]$

These formulae are derived in Appendix F.

\subsection{POAM expansions}

The input/output gains of the unaberrated DF are

$D_{l}^{-k}(r, \rho) \rightarrow \mathbf{D}(r, \rho ; \pm k) \delta_{k, l}=2 \int_{0}^{1} \mathrm{~d} s s J_{k}\left(2 \pi \kappa \frac{R_{\mathrm{tel}}}{\Theta} r s\right) J_{l}\left(2 \pi \kappa R_{\mathrm{tel}} \rho s\right) \delta_{k, l}$.

These gains are called the principal DF POAM functions, because they are also used in the input and output sensitivities

$D^{-k}(\overrightarrow{\mathbf{r}}, \rho) \rightarrow \mathbf{D}(r, \rho ; k) \mathrm{e}^{j k \xi}$

and

$D_{k}(r, \overrightarrow{\boldsymbol{\Omega}}) \rightarrow \mathbf{D}(r, \rho ; k) \mathrm{e}^{-j k \phi}$,

respectively (Appendix F). For an unaberrated telescope, the three DF POAM expansions in Table 1 collapse into one because of the Kronecker delta function,

$E(\overrightarrow{\mathbf{r}} ; t)=\sum_{p=-\infty}^{\infty} E_{p}(\rho ; t) \mathrm{e}^{j p \xi}=\sum_{p=-\infty}^{\infty}\left[2 \pi \int_{0}^{\infty} \mathrm{d} \rho \rho \mathbf{D}(r, \rho ; p) E_{p}(\rho ; t)\right] \mathrm{e}^{j p \xi}$

The first four principal DF POAM functions appear in Fig. 2. The abscissas are the scaled radii $\alpha$ and $\beta$. Since these variables are arguments to the same Bessel function of order $k$, the plots exhibit symmetry about $\alpha=\beta$. For $|k|>0$, there is a null that increases in depth and radius with increasing $|k|$. Decaying, concentric rings surround these nulls.

The unaberrated PSF sensitivities and gains can be discussed in terms of two types of principal PSF POAM functions (\#1 and \#2),

$\mathbf{P}(r, \rho ; \pm k, \pm l)=\mathbf{P}(r, \rho ; \pm k, \mp l)=\mathbf{D}(r, \rho ; k) \mathbf{D}(r, \rho ; l)$

and

$\mathbf{P}(r, \rho ; \pm l)=\sum_{k=-\infty}^{\infty} \mathbf{P}(r, \rho ; \pm k, \pm k \pm l)=\sum_{k=-\infty}^{\infty} \mathbf{P}(r, \rho ; \pm k, \pm k \mp l)$,

both of which are functions of the principal DF POAM components (Appendix F). For an unaberrated telescope, the eight PSF POAM expansions in Table 3 collapse into four,

$$
\begin{aligned}
& I(\overrightarrow{\mathbf{r}})=\sum_{p=-\infty}^{\infty} \sum_{q=-\infty}^{\infty}\left[2 \pi \int_{0}^{\infty} \mathrm{d} \rho \rho \mathbf{P}(r, \rho ; p-q) I_{p, q}(\rho)\right] \mathrm{e}^{j(p-q) \xi}, \\
& I(\overrightarrow{\mathbf{r}})=\sum_{p=-\infty}^{\infty} \sum_{q=-\infty}^{\infty}\left[2 \pi \int_{0}^{\infty} \mathrm{d} \rho \rho \mathbf{P}(r, \rho ; p, q) \mathcal{I}_{p-q}(\rho)\right] \mathrm{e}^{j(p-q) \xi}, \\
& I(\overrightarrow{\mathbf{r}})=\sum_{p=-\infty}^{\infty}\left[2 \pi \int_{0}^{\infty} \mathrm{d} \rho \rho \mathbf{P}(r, \rho ; p) \mathcal{I}_{p}(\rho)\right] \mathrm{e}^{j p \xi},
\end{aligned}
$$

and

$I(\overrightarrow{\mathbf{r}})=\sum_{p=-\infty}^{\infty}\left[\sum_{k=-\infty}^{\infty} 2 \pi \int_{0}^{\infty} \mathrm{d} \rho \rho \mathbf{P}(r, \rho ; p) I_{k, k-p}(\rho)\right] \mathrm{e}^{j p \xi}$. 

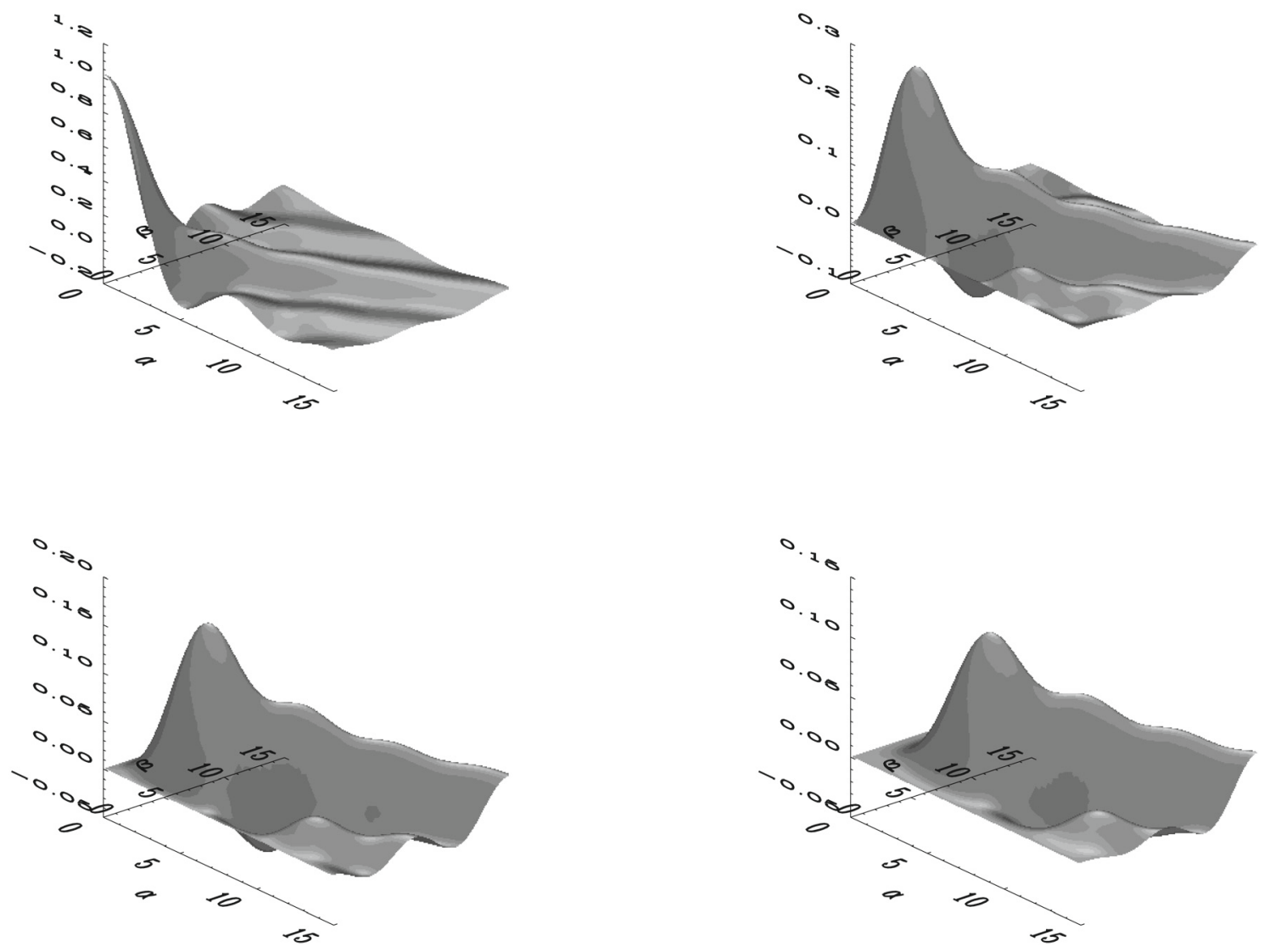

Fig. 2. The first four principal POAM DF functions $\left(k=0,1,2,3 \rightarrow\right.$ upper left, upper right, lower left, lower right). The abscissas are $\alpha=2 \pi \kappa \frac{R_{\mathrm{tel}}}{\Theta} r$ and $\beta=2 \pi \kappa R_{\mathrm{tel}} \rho$.
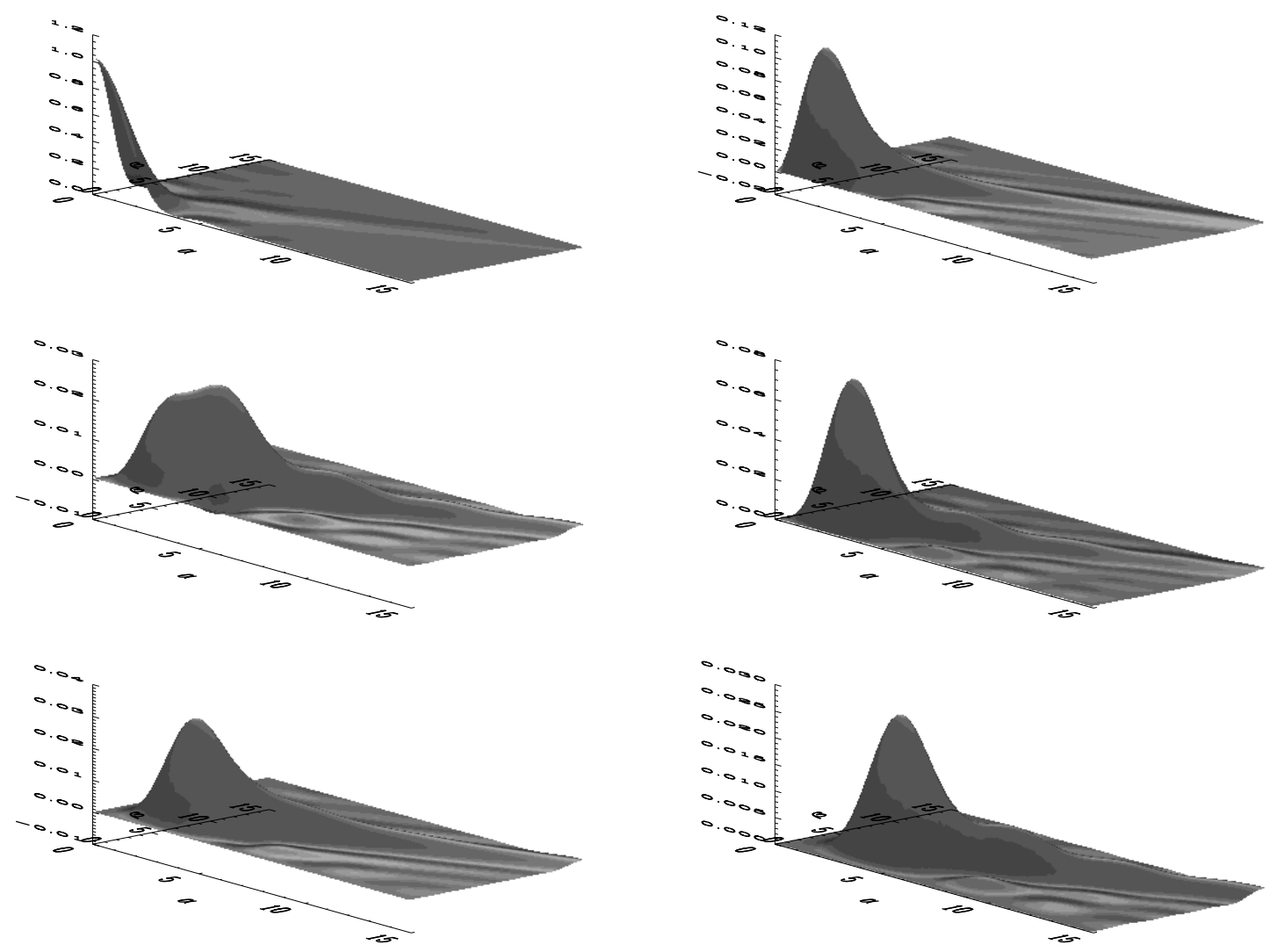

Fig. 3. The first six principal POAM PSF functions \#1 $[(p, q)=(0,0),(0,1),(0,2),(1,1),(1,2),(2,2) \rightarrow$ upper left, upper right, middle left, middle right, lower left, lower right]. The abscissas are $\alpha=2 \pi \kappa \frac{R_{\mathrm{tel}}}{\Theta} r$ and $\beta=2 \pi \kappa R_{\mathrm{tel}} \rho$. 

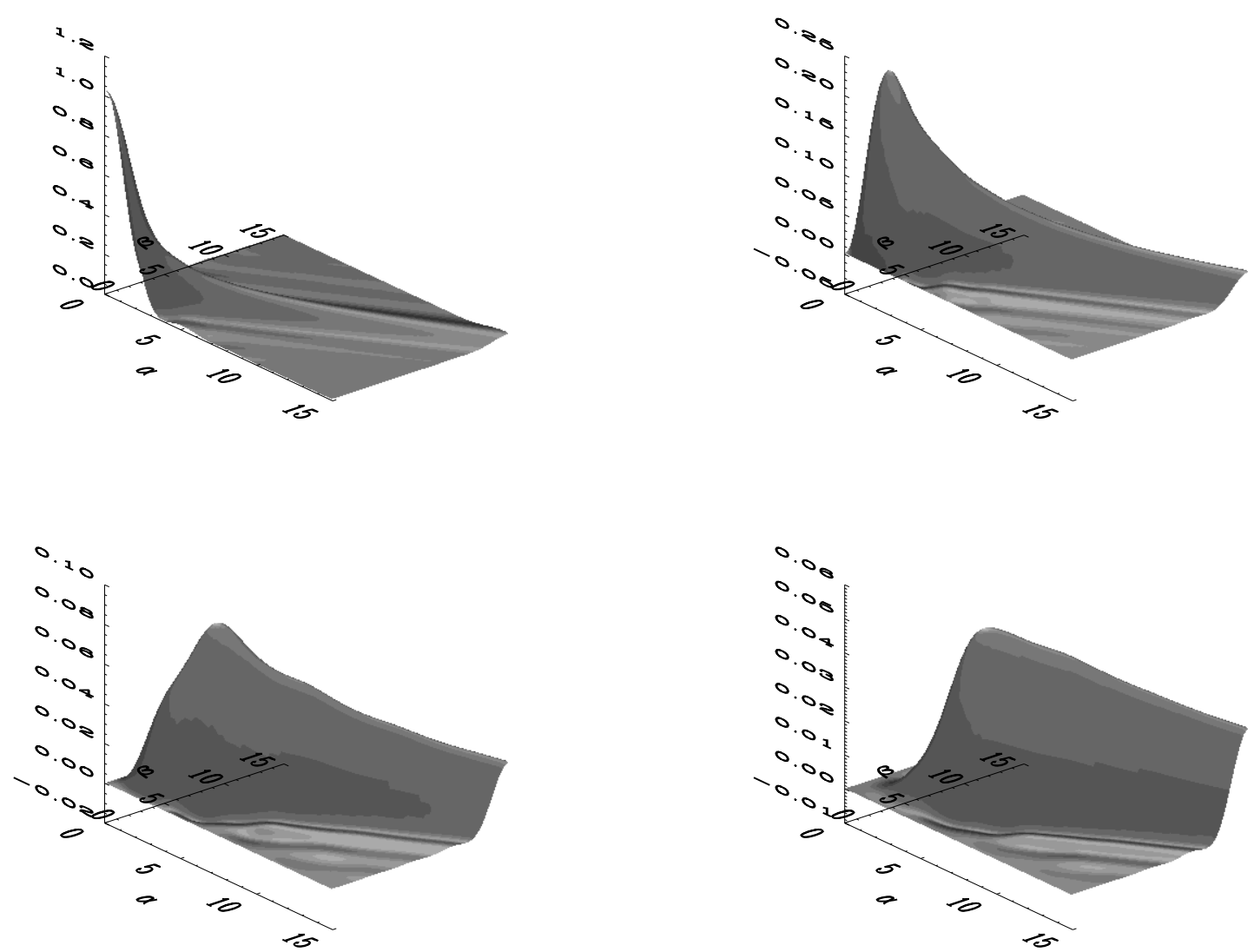

Fig. 4. The first four principal POAM PSF functions \#2 ( $p=0,1,2,3 \rightarrow$ upper left, upper right, lower left, lower right). The abscissas are $\alpha=$ $2 \pi \kappa \frac{R_{\mathrm{tel}}}{\Theta} r$ and $\beta=2 \pi \kappa R_{\mathrm{tel}} \rho$.

The first six principal PSF POAM functions of the first type are presented in Fig. 3. Also, the first four principal PSF POAM functions of the second type are presented in Fig. 4. All plots employ the normalized radii $\alpha$ and $\beta$, and they are symmetric about $\alpha$ $=\beta$. For $|k|,|l|>0$, there is a null that increases in depth and radius with increasing $|k|,|l|$.

According to Eq. (27d), each state on the celestial sphere produces only the same state in the image plane. Similarly, Eqs. (29a,c) shows that each correlation (rancor) on the celestial sphere produces only the same correlation (rancor) in the image plane. The radial distributions, however, have changed. Propagation through an unaberrated telescope, like propagation through free space (Sect. 5), applies no external torque to the wavefronts. Therefore, the integrated probabilities and the POAM expectation value on the celestial sphere and in the image plane are conserved quantities, or $p_{p, p}^{i p}=p_{p, p}^{\mathrm{cs}}$ and $\hat{L}_{Z}^{i p}=\hat{L}_{Z}^{\mathrm{cs}}$. In Appendix F, I prove this assertion using different mathematics.

\section{POAM propagation through a focal-plane coronagraph}

A basic coronagraph consists of a telescope, an image-plane mask, and filtering/reimaging optics (Fig. 5). The telescope is identical to the one described in Sect. 6. Wavefront control is important for coronagraphs, so a deformable mirror could be located in the telescope exit pupil (TEP). The mask is placed in the telescope image plane (TIP; the image plane of Sect. 6). From the TIP, the light passes through the reimage exit pupil (REP) to the reimage plane (RIP). Apodization optics for filtering the higher spatial frequencies within the REP, including masks such as Lyot stops, can be used to improve instrument performance. I will not consider any pupil-plane masks. In this section, I 1) form the DF and the POAM expansions; 2) show that the input/output gains of an unaberrated system can be expressed in terms of the principal DF POAM functions of the telescope and reimaging system and the azimuthal DFT of the mask; 3) demonstrate via POAM why a null occurs; and 4) plot the responses of alternating and vortex phase masks. Anzolin et al. (2008) have recently performed coronagraphy using a related technique, modulating the POAM of a central star with a fork hologram.

\subsection{Classical forms}

In a focal-plane coronagraph, the TIP electric field (Eq. (23a)) is modified by a mask function $M(\overrightarrow{\mathbf{r}}, \overrightarrow{\mathbf{\Omega}})$, or

$E^{\prime}(\overrightarrow{\mathbf{r}}, \overrightarrow{\mathbf{\Omega}} ; t)=M(\overrightarrow{\mathbf{r}}, \overrightarrow{\mathbf{\Omega}}) E(\overrightarrow{\mathbf{r}}, \overrightarrow{\mathbf{\Omega}} ; t)=M(\overrightarrow{\mathbf{r}}, \overrightarrow{\mathbf{\Omega}}) D(\overrightarrow{\mathbf{r}}, \overrightarrow{\mathbf{\Omega}}) E(\overrightarrow{\mathbf{\Omega}} ; t)$ 
reimaging system

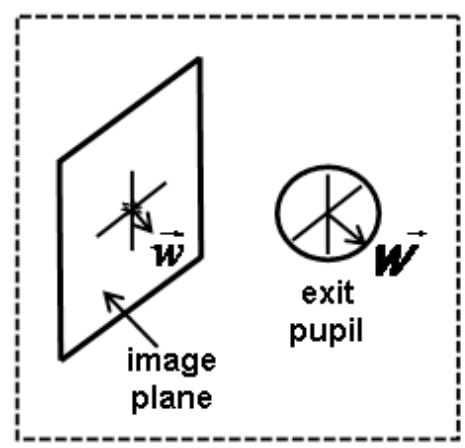

telescope

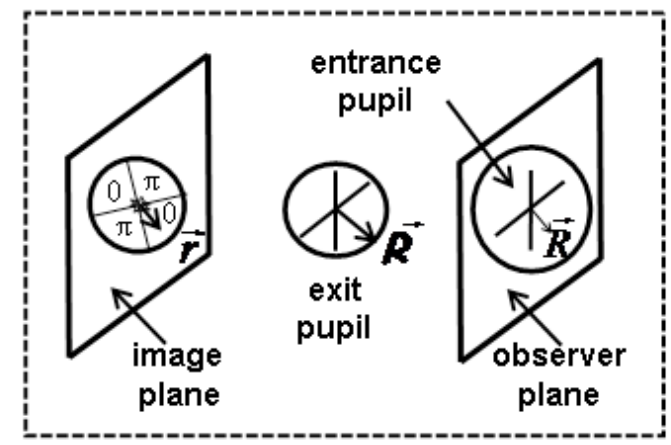

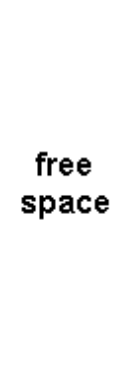

Fig. 5. A coronagraph schematic diagram from a source on the celestial sphere to the reimage plane through an image-plane mask. The complete system consists of two subsystems, telescope and reimaging optics.

where $D(\overrightarrow{\mathbf{r}}, \overrightarrow{\mathbf{\Omega}})$ is the telescope DF (TDF) derived in Sect. 6. The electric field then propagates to the REP

$E(\overrightarrow{\mathcal{W}}, \overrightarrow{\boldsymbol{\Omega}} ; t)=\int \mathrm{d}^{2} r \mathrm{e}^{j 2 \pi \kappa \frac{\overrightarrow{\mathrm{r}}}{f} \cdot \overrightarrow{\mathcal{W}}} \mathcal{D}(\overrightarrow{\mathcal{W}}, \overrightarrow{\mathbf{r}}, \overrightarrow{\boldsymbol{\Omega}}) E^{\prime}(\overrightarrow{\mathbf{r}}, \overrightarrow{\boldsymbol{\Omega}} ; t)=\int \mathrm{d}^{2} r \mathrm{e}^{j 2 \pi \kappa \frac{\vec{r}}{f} \cdot \overrightarrow{\mathcal{W}}} \mathcal{D}(\overrightarrow{\mathcal{W}}, \overrightarrow{\mathbf{r}}, \overrightarrow{\boldsymbol{\Omega}}) M(\overrightarrow{\mathbf{r}}, \overrightarrow{\boldsymbol{\Omega}}) D(\overrightarrow{\mathbf{r}}, \overrightarrow{\boldsymbol{\Omega}}) E(\overrightarrow{\boldsymbol{\Omega}} ; t)$,

where $\overrightarrow{\mathcal{W}}=\left(\mathcal{X}_{r}, \mathcal{Y}_{r}\right)=(\mathcal{W} \cos \eta, \mathcal{W} \sin \eta)$ is the vector in the REP, $\mathcal{X}_{r}$ and $\mathcal{Y}_{r}$ are the Cartesian coordinates, $\mathcal{W}$ is the radial coordinate, $\eta$ is the azimuthal coordinate, $f$ is the focal length between the TIP and REP (also between the TEP and TIP), and $\mathcal{D}(\overrightarrow{\mathcal{W}}, \overrightarrow{\mathbf{r}}, \overrightarrow{\boldsymbol{\Omega}})$ is the reimage amplitude/phase aberration function (RAF) projected into the REP. The RAF can also contain additional spatial filtering. Finally, the electric field reaches the RIP

$E(\overrightarrow{\mathbf{w}}, \overrightarrow{\boldsymbol{\Omega}} ; t)=\frac{1}{A_{\text {rep }}} \int \mathrm{d}^{2} \mathcal{W} \mathrm{e}^{-j 2 \pi \kappa \frac{\vec{r}}{r_{r}} \cdot \vec{W}} E(\overrightarrow{\mathcal{W}}, \overrightarrow{\boldsymbol{\Omega}} ; t)=\left[\int \mathrm{d}^{2} r D(\overrightarrow{\mathbf{w}}, \overrightarrow{\mathbf{r}}, \overrightarrow{\boldsymbol{\Omega}}) M(\overrightarrow{\mathbf{r}}, \overrightarrow{\boldsymbol{\Omega}}) D(\overrightarrow{\mathbf{r}}, \overrightarrow{\boldsymbol{\Omega}})\right] E(\overrightarrow{\boldsymbol{\Omega}} ; t)=D(\overrightarrow{\mathbf{w}}, \overrightarrow{\boldsymbol{\Omega}}) E(\overrightarrow{\boldsymbol{\Omega}} ; t)$,

where $\overrightarrow{\mathbf{w}}=\left(w_{x}, w_{y}\right)=(w \cos \omega, w \sin \omega)$ is the vector in the RIP, $w_{x}$ and $w_{y}$ are the Cartesian coordinates, $w$ is the radial coordinate, $\omega$ is the azimuthal coordinate, $A_{\text {rep }}$ is the REP area, $f_{r}$ is the focal length between the REP and RIP,

$D(\overrightarrow{\mathbf{w}}, \overrightarrow{\mathbf{r}}, \overrightarrow{\boldsymbol{\Omega}})=\frac{1}{A_{\text {rep }}} \int \mathrm{d}^{2} \mathcal{W} \mathrm{e}^{-j 2 \pi\left(\frac{\overrightarrow{\mathrm{w}}}{f_{r}}-\frac{\vec{r}}{f}\right) \cdot \overrightarrow{\mathcal{W}}} \mathcal{D}(\overrightarrow{\mathcal{W}}, \overrightarrow{\mathbf{r}}, \overrightarrow{\boldsymbol{\Omega}})=\frac{1}{A_{\text {rep }}} \int \mathrm{d}^{2} \mathcal{W} \mathrm{e}^{-j 2 \pi k M\left(\frac{\overrightarrow{\mathrm{w}}}{\mathrm{\theta}_{r}}-\frac{\overrightarrow{\mathrm{r}}}{\Theta}\right) \cdot \overrightarrow{\mathcal{W}}} \mathcal{D}(\overrightarrow{\mathcal{W}}, \overrightarrow{\mathbf{r}}, \overrightarrow{\boldsymbol{\Omega}})$

is the reimage $\mathrm{DF}$ (RDF), $\Theta=M f$ is the plate scale relating the coordinates on the celestial sphere to the TIP, $M$ is the magnification of the coordinates within the entrance pupil and the coordinates within the REP, $\Theta_{r}=M_{r} \Theta$ is the plate scale relating the coordinates in the TIP to the RIP, $M_{r}=f_{r} / f$ is the relative magnification between the TIP and RIP, and $D(\overrightarrow{\mathbf{w}}, \overrightarrow{\mathbf{\Omega}})$ is the coronagraph DF (CDF) of the telescope and reimaging system. The overlap integrals,

$E(\overrightarrow{\mathbf{w}} ; t)=\int \mathrm{d}^{2} \Omega D(\overrightarrow{\mathbf{w}}, \overrightarrow{\boldsymbol{\Omega}}) E(\overrightarrow{\boldsymbol{\Omega}} ; t)$

and

$I(\overrightarrow{\mathbf{w}})=\int \mathrm{d}^{2} \Omega P(\overrightarrow{\mathbf{w}}, \overrightarrow{\boldsymbol{\Omega}}) I(\overrightarrow{\boldsymbol{\Omega}})=\int \mathrm{d}^{2} \Omega|D(\overrightarrow{\mathbf{w}}, \overrightarrow{\boldsymbol{\Omega}})|^{2}\left\langle\frac{1}{2}|E(\overrightarrow{\mathbf{w}} ; t)|^{2}\right\rangle$,

are expressed in terms of the CDF and coronagraph PSF (CPSF) and have the same forms as in Sect. $4(\overrightarrow{\mathbf{N}} \rightarrow \overrightarrow{\mathbf{w}}$ and $\overrightarrow{\mathbf{a}} \rightarrow \overrightarrow{\mathbf{0}})$.

In general, the mask function weakly depends on $\overrightarrow{\boldsymbol{\Omega}}$ because masks have a finite thickness. Also, the RAF and RDF are weak functions of $\overrightarrow{\boldsymbol{\Omega}}$ due to aberrations. Assuming an unaberrated TDF, an unaberrated RDF, and a mask and RDF that are independent of position on the celestial sphere, the CDF becomes

$D(\overrightarrow{\mathbf{w}}, \overrightarrow{\boldsymbol{\Omega}})=\int \mathrm{d}^{2} r \mathcal{J}_{1}\left[2 \pi \kappa R_{\mathrm{tel}} \Gamma(\overrightarrow{\mathbf{w}}, \overrightarrow{\mathbf{r}})\right] M(\overrightarrow{\mathbf{r}}) \mathcal{J}_{1}\left[2 \pi \kappa R_{\mathrm{tel}} \Gamma(\overrightarrow{\mathbf{r}}, \overrightarrow{\boldsymbol{\Omega}})\right]$,

where

$\Gamma(\overrightarrow{\mathbf{w}}, \overrightarrow{\mathbf{r}})=\left|\frac{\overrightarrow{\mathbf{w}}}{\Theta_{r}}-\frac{\overrightarrow{\mathbf{r}}}{\Theta}\right|$

and

$\Gamma(\overrightarrow{\mathbf{r}}, \overrightarrow{\boldsymbol{\Omega}})=\left|\frac{\overrightarrow{\mathbf{r}}}{\Theta}-\overrightarrow{\mathbf{s}}_{x y}(\overrightarrow{\boldsymbol{\Omega}})\right|$ 
are the special coordinates, $R_{\mathrm{tel}}=M R_{\mathrm{tep}}$ is the radius of the telescope (entrance pupil), and $R_{\text {tep }}$ is the radius of the TEP. As a check, if I let $M(\overrightarrow{\mathbf{r}})=1$ (a trivial non-coronagraphic mask) and allow the integration range within the TIP to be large, the CDF becomes

$D(\overrightarrow{\mathbf{w}}, \overrightarrow{\mathbf{\Omega}}) \rightarrow \mathcal{D}_{0} \mathcal{J}_{1}\left[2 \pi \kappa R_{\mathrm{tel}} \Gamma(\overrightarrow{\mathbf{w}}, \overrightarrow{\mathbf{\Omega}})\right]$

where

$\Gamma(\overrightarrow{\mathbf{w}}, \overrightarrow{\mathbf{\Omega}})=\left|\frac{\overrightarrow{\mathbf{w}}}{\Theta_{r}}-\overrightarrow{\mathbf{s}}_{x y}(\overrightarrow{\mathbf{\Omega}})\right|$

is another special coordinate. The corresponding CPSF simply becomes the Airy pattern, or

$P(\overrightarrow{\mathbf{w}}, \overrightarrow{\mathbf{\Omega}})=|D(\overrightarrow{\mathbf{w}}, \overrightarrow{\mathbf{\Omega}})|^{2} \rightarrow \mathcal{D}_{0}^{2} \mathcal{J}_{1}^{2}\left[2 \pi \kappa R_{\text {tel }} \Gamma(\overrightarrow{\mathbf{w}}, \overrightarrow{\mathbf{\Omega}})\right]=\mathcal{P}_{0} \mathcal{A}\left[2 \pi \kappa R_{\text {tel }} \Gamma(\overrightarrow{\mathbf{w}}, \overrightarrow{\boldsymbol{\Omega}})\right]$

Since the reimaging system does not act like a coronagraph, a scaled replica of the TIP image appears in the RIP. No external torque is applied to the wavefront, so the integrated probabilities and the POAM expectation value are conserved. Conversely, inserting a coronagraphic mask must apply external torque, changing the integrated probabilities and POAM expectation value.

\subsection{POAM expansions}

To demonstrate how a non-trivial TIP mask affects the POAM spectrum and how it produces a null at the FOV center, I first calculate the POAM expansions of the CDF. When the TDF and RDF are expanded in terms of the TIP sensitivities, the CDF becomes

$D(\overrightarrow{\mathbf{w}}, \overrightarrow{\boldsymbol{\Omega}})=\sum_{k=-\infty}^{\infty} \sum_{p=-\infty}^{\infty} 2 \pi \int_{0}^{r_{\text {tip }}} \mathrm{d} r r D^{-k}(\overrightarrow{\mathbf{w}}, r, \overrightarrow{\boldsymbol{\Omega}}) M_{k-p}(r, \overrightarrow{\boldsymbol{\Omega}}) D_{p}(r, \overrightarrow{\boldsymbol{\Omega}})$,

where $r_{\text {tip }}$ is the unocculted radius of the TIP, and

$M(\overrightarrow{\mathbf{r}}, \overrightarrow{\boldsymbol{\Omega}})=\sum_{g=-\infty}^{\infty} M_{g}(r, \overrightarrow{\boldsymbol{\Omega}}) \mathrm{e}^{j g \xi} \stackrel{\mathcal{F}}{\Leftrightarrow} M_{g}(r, \overrightarrow{\boldsymbol{\Omega}})=\frac{1}{2 \pi} \int_{0}^{2 \pi} \mathrm{d} \xi \mathrm{e}^{-j g \xi} M(\overrightarrow{\mathbf{r}}, \overrightarrow{\boldsymbol{\Omega}})$

is the TIP Fourier expansion of the mask. Assuming a mask and RDF that are independent of position on the celestial sphere, I express the CDF input/output gains as a function of the TDF and RDF input/output gains,

$$
\begin{aligned}
D(\overrightarrow{\mathbf{w}}, \overrightarrow{\boldsymbol{\Omega}})=\sum_{u=-\infty}^{\infty} \sum_{m=-\infty}^{\infty} D_{u}^{-m}(w, \rho) \mathrm{e}^{-j m \phi} \mathrm{e}^{j u \omega} \stackrel{\mathcal{F F}}{\Leftrightarrow} D_{u}^{-m}(w, \rho) & =\frac{1}{2 \pi} \int_{0}^{2 \pi} \mathrm{d} \omega \mathrm{e}^{-j u \omega} \frac{1}{2 \pi} \int_{0}^{2 \pi} \mathrm{d} \phi \mathrm{e}^{j m \phi} D(\overrightarrow{\mathbf{w}}, \overrightarrow{\boldsymbol{\Omega}}) \\
& =\sum_{k=-\infty}^{\infty} \sum_{p=-\infty}^{\infty} 2 \pi \int_{0}^{r_{\mathrm{tip}}} \mathrm{d} r r D_{u}^{-k}(w, r) M_{k-p}(r) D_{p}^{-m}(r, \rho) .
\end{aligned}
$$

If the telescope and reimaging system are unaberrated, then

$D_{u}^{-m}(w, \rho)=2 \pi \int_{0}^{r_{\text {tip }}} \mathrm{d} r r \mathbf{D}_{u}^{-u}(w, r) M_{u-m}(r) \mathbf{D}_{m}^{-m}(r, \rho)=2 \pi \int_{0}^{r_{\text {tip }}} \mathrm{d} r r \mathbf{D}(w, r ; u) M_{u-m}(r) \mathbf{D}(r, \rho ; m)$,

where the

$D_{u}^{-k}(w, r) \rightarrow \mathbf{D}(w, r ; \pm u) \delta_{k, u}=2 \int_{0}^{1} \mathrm{~d} s s J_{u}\left(2 \pi \kappa R_{\mathrm{tel}} \frac{w}{\Theta_{r}} s\right) J_{u}\left(2 \pi \kappa R_{\mathrm{tel}} \frac{r}{\Theta} s\right) \delta_{k, u}$

are the RDF input/output gains, and the

$D_{p}^{-m}(r, \rho) \rightarrow \mathbf{D}(r, \rho ; \pm m) \delta_{p, m}=2 \int_{0}^{1} \mathrm{~d} s s J_{m}\left(2 \pi \kappa R_{\mathrm{tel}} \frac{r}{\Theta} s\right) J_{m}\left(2 \pi \kappa R_{\mathrm{tel}} \rho s\right) \delta_{p, m}$

are the TDF input/output gains. Because the telescope and reimaging system are unaberrated, these input/output gains are principal DF POAM components (Eq. (27a)), causing the double sum in the CDF to disappear. Understanding the basic POAM behavior of focal-plane coronagraphs is now simply a matter of substituting different masks into Eqs. (35) or (36a) and reconstructing the CDF and CPSF. 


\subsection{Sector masks}

The central star of a planetary system contributes most of the zero-order state to the scene. To demonstrate how focal-plane coronagraphs attenuate low-order states, I employ an alternating phase sector mask and a vortex sector mask.

Continuous azimuthally-dependent masks are often approximated by sectors because they are easier to manufacture

$M(\overrightarrow{\mathbf{r}}, \overrightarrow{\mathbf{\Omega}})=\sum_{g=0}^{2 N-1} m_{g}(r, \overrightarrow{\boldsymbol{\Omega}}) p\left(\xi-\frac{2 \pi g}{2 N}, \frac{\pi}{N}\right)$

where $2 N=2,4,6, \ldots$ is the number of sectors (even only), the $m_{g}(r, \overrightarrow{\boldsymbol{\Omega}})$ are the complex sector weights (magnitudes between 0 and 1$)$, and $p(\theta, \Delta \theta)$ is a pulse function centered at $\theta=0$ of width $\Delta \theta$. Masks that depend on azimuth modulate the states. I will not consider radial apodization in this work, since it serves only to tweak coronagraph performance. The TIP Fourier components of the sector mask are

$M_{h}(r, \overrightarrow{\boldsymbol{\Omega}})=\mathcal{M}_{h}(r, \overrightarrow{\boldsymbol{\Omega}}) \operatorname{sinc}\left(\pi \frac{h}{2 N}\right)$,

where the

$\mathcal{M}_{h}(r, \overrightarrow{\boldsymbol{\Omega}})=\frac{1}{2 N} \sum_{g=0}^{2 N-1} \mathrm{e}^{-j 2 \pi \frac{h g}{2 N}} m_{g}(r, \overrightarrow{\boldsymbol{\Omega}})$

are the Discrete Fourier Transform (DFT) components of the mask weights (the unapodized TIP Fourier components of the mask). The DFT is periodic in $2 N$. Therefore, all mask Fourier components $-\infty<h<\infty$ influence how the mask works, limited only by the $\operatorname{sinc}(\cdot)$ function arising directly from the sector approximation. I derive this result in Appendix G. Substituting Eq. (37b) into Eqs. (35) and (36a), I obtain the aberrated

$D_{u}^{-m}(w, \rho)=\sum_{k=-\infty}^{\infty} \sum_{p=-\infty}^{\infty} 2 \pi \int_{0}^{r_{\text {tip }}} \mathrm{d} r r D_{u}^{-k}(w, r) \mathcal{M}_{k-p}(r) D_{p}^{-m}(r, \rho) \operatorname{sinc}\left(\pi \frac{k-p}{2 N}\right)$

and unaberrated

$D_{u}^{-m}(w, \rho)=2 \pi \int_{0}^{r_{\text {tip }}} \mathrm{d} r r \mathbf{D}(w, r ; u) \mathcal{M}_{u-m}(r) \mathbf{D}(r, \rho ; m) \operatorname{sinc}\left(\pi \frac{u-m}{2 N}\right)$

$\mathrm{CDF}$ input/output gains for sector masks. From these equations, it is clear that the sector mask Fourier components and the sinc $(\cdot)$ function attenuate or eliminate some principal TDF and RDF POAM components.

An alternating mask uses azimuthal $r$-independent sectors whose phases alternate between $0^{\circ}$ and $180^{\circ}$,

$m_{g}(r) \rightarrow m_{g}=\mathrm{e}^{j g \pi}$.

Rouan et al. (2000), Riaud et al. (2001), Boccaletti et al. (2002), Riaud et al. (2003), and Boccaletti et al. (2004) have studied the $2 N=4$ mask extensively, but here I discuss an arbitrary number of sectors. The sector mask Fourier components (Appendix G) are

$\mathcal{M}_{h}(r) \rightarrow \mathcal{M}_{h}=\sum_{l=-\infty}^{\infty} \delta_{h,(2 l+1) N}$,

which means that the aberrated and unaberrated CDF input/output gains become

$$
\begin{aligned}
D_{u}^{-m}(w, \rho) & =\sum_{k=-\infty}^{\infty} \sum_{p=-\infty}^{\infty} 2 \pi \int_{0}^{r_{\mathrm{tip}}} \mathrm{d} r r D_{u}^{-k}(w, r) D_{p}^{-m}(r, \rho)\left\{\sum_{l=-\infty}^{\infty} \delta_{k-p,(2 l+1) N} \operatorname{sinc}\left[\pi\left(l+\frac{1}{2}\right)\right]\right\} \\
& =\sum_{k=-\infty}^{\infty} \sum_{p=-\infty}^{\infty} 2 \pi \int_{0}^{r_{\mathrm{tip}}} \mathrm{d} r r D_{u}^{-k}(w, r) D_{p}^{-m}(r, \rho) \mathcal{G}_{a}(k-p ; N)
\end{aligned}
$$

and

$D_{u}^{-m}(w, \rho)=2 \pi \int_{0}^{r_{\mathrm{tip}}} \mathrm{d} r r \mathbf{D}(w, r ; u) \mathbf{D}(r, \rho ; m) \mathcal{G}_{a}(u-m ; N)$,

where $\mathcal{G}_{a}(i ; N)$ is the alternating-phase modulation function. We know from Eq. (36c) that the only TDF input/output gain that yields significant values near the FOV center is $\mathbf{D}(r, \rho \approx 0 ; 0)$. Since the RDF input/output gain has the same functional form, the only one that yields significant values near the FOV center is $\mathbf{D}(w, r \approx 0 ; 0)$. According to Eq. (40b), the unaberrated CDF input/output gain cannot pass light near the FOV center when both $u$ and $m$ are zero because $\mathcal{G}_{a}(0 ; N)=0$. Therefore, the instrument does indeed act as a coronagraph. 
A vortex mask employs azimuthal $r$-independent sectors whose phases approximate a single vortex,

$m_{g}(r) \rightarrow m_{g}=\mathrm{e}^{j 2 \pi \frac{N_{w} g}{2 N}}$

where $N_{w}$ is the number of phase wraps per $360^{\circ}$. This vortex sector mask is identical to the alternating sector mask for $N_{w}=N$. Lee et al. (2006) have studied the $N_{w}=2$ sector mask, but here I discuss an arbitrary value. The sector mask Fourier components (Appendix G) are

$\mathcal{M}_{h}(r) \rightarrow \mathcal{M}_{h}=\sum_{l=-\infty}^{\infty} \delta_{h, 2 l N+N_{w}}$

which means that the aberrated and unaberrated CDF input/output gains become

$$
\begin{aligned}
D_{u}^{-m}(w, \rho) & =\sum_{k=-\infty}^{\infty} \sum_{p=-\infty}^{\infty} 2 \pi \int_{0}^{r_{\mathrm{tip}}} \mathrm{d} r r D_{u}^{-k}(w, r) D_{p}^{-m}(r, \rho)\left\{\sum_{l=-\infty}^{\infty} \delta_{k-p, 2 l N+N_{w}} \operatorname{sinc}\left[\pi\left(l+\frac{1}{2} \frac{N_{w}}{N}\right)\right]\right\} \\
& =\sum_{k=-\infty}^{\infty} \sum_{p=-\infty}^{\infty} 2 \pi \int_{0}^{r_{\mathrm{tip}}} \mathrm{d} r r D_{u}^{-k}(w, r) D_{p}^{-m}(r, \rho) \mathcal{G}_{v}\left(k-p ; N, N_{w}\right)
\end{aligned}
$$

and

$D_{u}^{-m}(w, \rho)=2 \pi \int_{0}^{r_{\text {tip }}} \mathrm{d} r r \mathbf{D}(w, r ; u) \mathbf{D}(r, \rho ; m) \mathcal{G}_{v}\left(u-m ; N, N_{w}\right)$,

where $\mathcal{G}_{v}\left(i ; N, N_{w}\right)$ is the vortex modulation function. This vortex sector mask forces the instrument to act as a coronagraph in the same manner as the alternating sector mask.

The number of numerically significant CDF input/output gains is large, so plotting all of them is somewhat prohibitive. In addition, they exhibit $\approx$ the same behavior, namely a null at the center of the RIP that attenuates the star light. Therefore, I present plots that display the overall CPSFs, combining all of the CDF/CPSF input/output gains.

The average intensity leakage for a point source at the FOV center is a simple performance metric for coronagraphs. It is defined as

$\bar{P}(w, \overrightarrow{\mathbf{0}})=\frac{1}{2 \pi} \int_{0}^{2 \pi} \mathrm{d} \omega P(\overrightarrow{\mathbf{w}}, \overrightarrow{\mathbf{0}})$.

This quantity conveniently eliminates $\omega$ in the RIP as a dependent variable. In Fig. 6, I show the intensity leakage for an alternating mask with $N=2,4,6$ (four, eight, or twelve alternating sectors). The normalized radial coordinate in the RIP is $\gamma$. The basic envelope (a line following the peaks) is $\approx$ the same for all $N$. The only major difference between the curves is that the depth and width of the null increases with $N$. In Fig. 7, I show the intensity leakage for an $N_{w}=2$ sector mask with $N=2,4,6$. The $N_{w}=N$ curve is identical to the $N=2$ curve in Fig. 6 . The other two curves in Fig. 7 are very similar (but not exactly the same) to the other two curves in Fig. 6.

The azimuthally averaged PSF for a coronagraph is

$\bar{P}(w, \rho)=\frac{1}{2 \pi} \int_{0}^{2 \pi} \mathrm{d} \omega \frac{1}{2 \pi} \int_{0}^{2 \pi} \mathrm{d} \phi P(\overrightarrow{\mathbf{w}}, \overrightarrow{\boldsymbol{\Omega}})$.

This quantity eliminates both azimuthal coordinates, providing a rough estimate for the throughput of possible planets as a function of radial coordinates. In Fig. 8, I show the azimuthally averaged PSFs for an alternating mask with $N=2$, 4, 6, as well as the difference between the $N=2$ and $N=6$ PSFs. I use the normalized radial coordinates $\alpha$ and $\gamma$. The increasing null width and depth increasing with $N$ is clearly evident. For each $\alpha$, there is a corresponding maximum for a single $\gamma$. Planets are attenuated, but much less than the central star.

\section{POAM Propagation through a Michelson interferometer}

Interferometers - which employ telescopes (Sect. 6), feed systems, delay lines, and a beam combiner - are used to sythesize an entrance pupil in the observation plane that is larger than a single telescope. In other words, by Fourier transforming a large number visibilities at different baseline vectors and processing the dirty image, it is possible to estimate the source intensity on the celestial sphere at high spatial resolution with an ensemble of relatively small telescopes. Also, a small number of interferometer observations can be used to model simple sources, e.g., measuring the angular diameter of a star or measuring the separation and position angle of a binary star.

Consider Fig. 9, where a wavefront enters the front end of an interferometer. If this wavefront is expanded into vortices about the midpoint between the telescope entrance pupils, the phase difference is $k \pi$ for each POAM state $k$. Therefore, interferometers are sensitive to diametrically opposed pieces of vortices at a spatial frequency proportional to the baseline length in the observation plane (perpendicular to the line of sight). The POAM response can be changed by moving the delay tracking point and/or the baseline vector. For example, single-Bracewell interferometers track $180^{\circ}$ from the central fringe to null light from a central star 


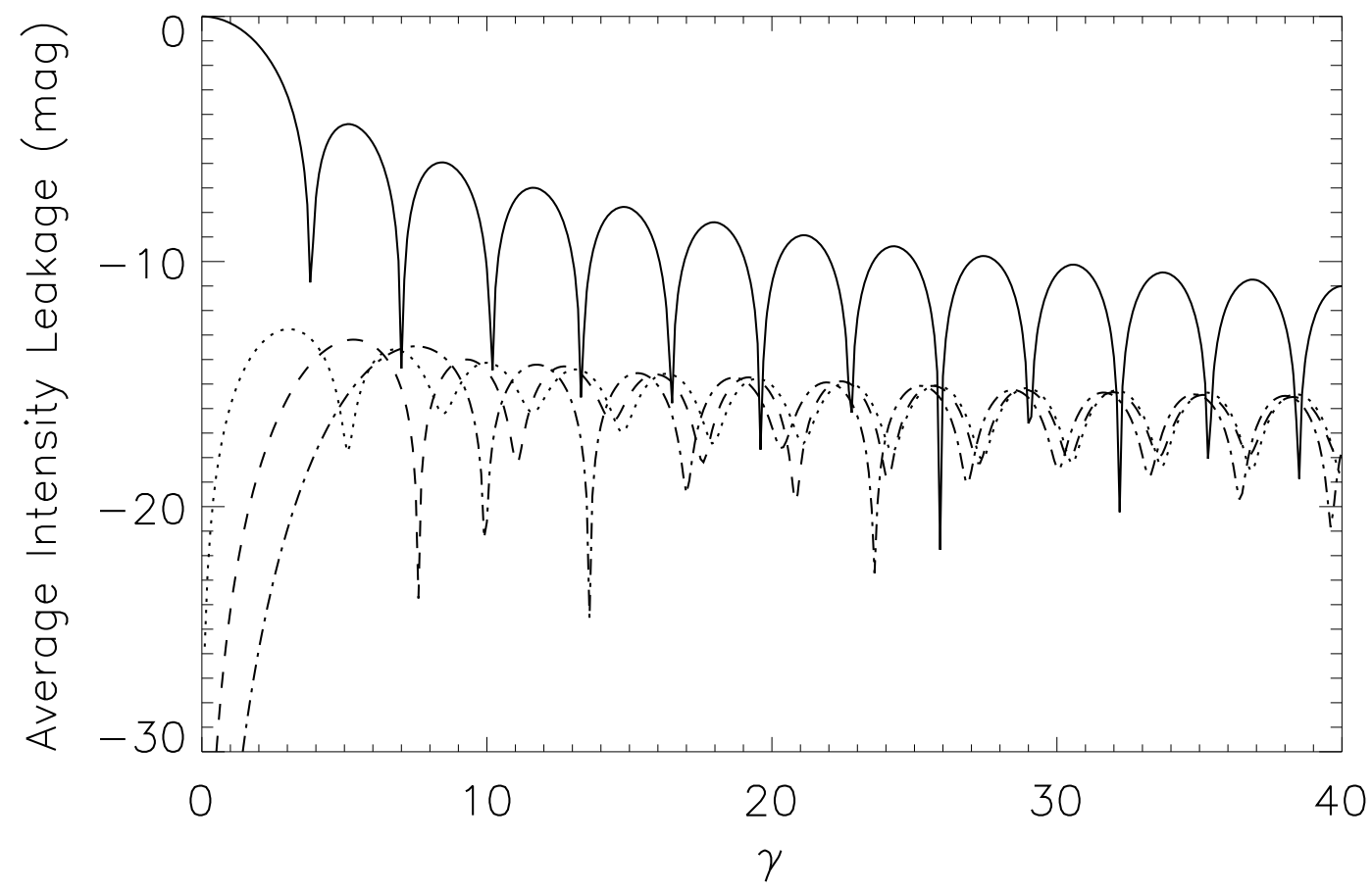

Fig. 6. The average intensity leakage (in magnitudes) versus $\gamma=2 \pi \kappa R_{\mathrm{tel}} w / \Theta_{r}$ for a coronagraph using an alternating mask. The solid curve corresponds to the Airy function (no mask). The dotted, dashed, and dot-dashed lines correspond to $N=2,4$, and 6 , respectively.

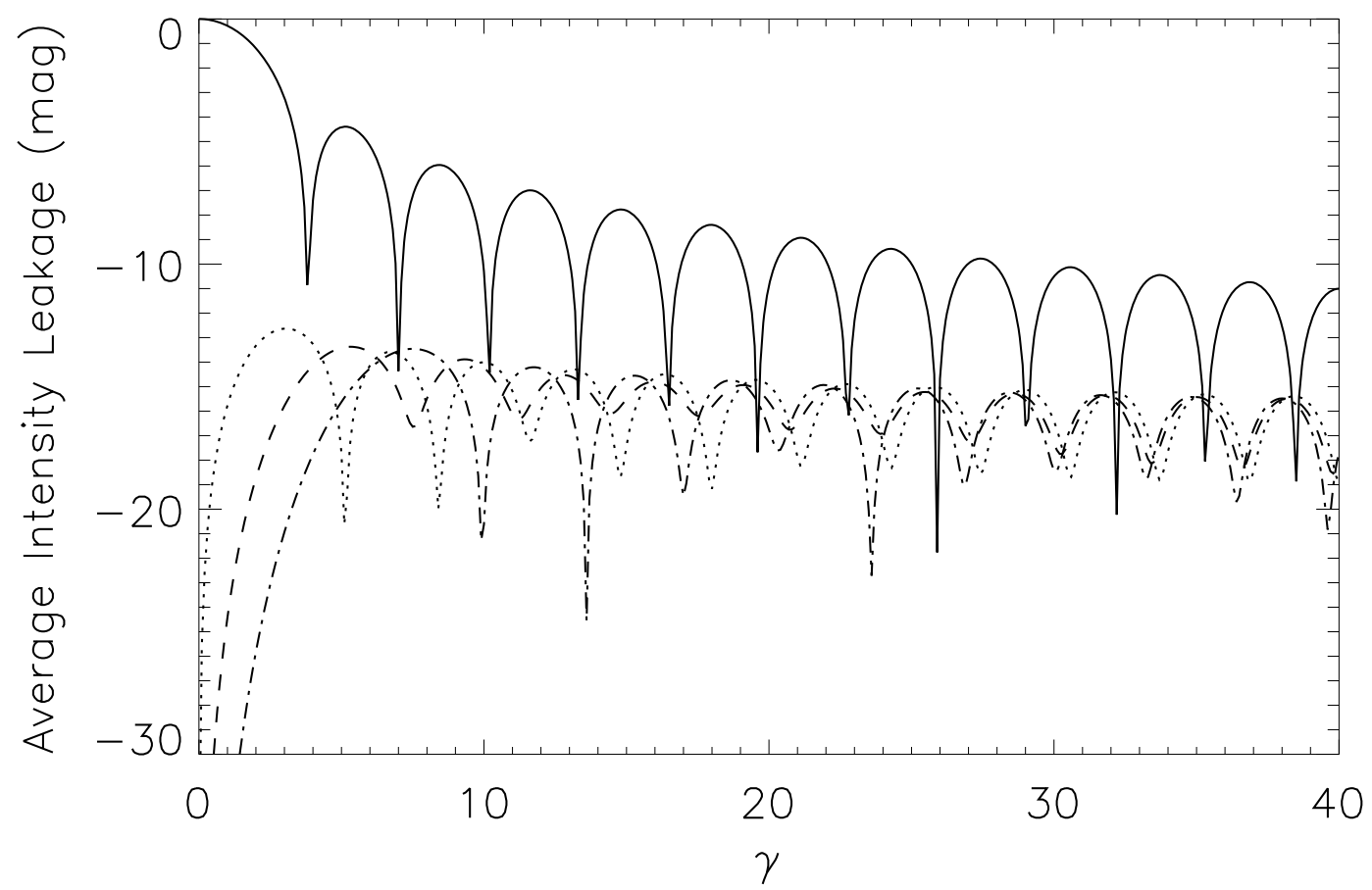

Fig. 7. The average intensity leakage (in magnitudes) versus $\gamma=2 \pi \kappa R_{\mathrm{tel}} w / \Theta_{r}$ for a coronagraph using an $N_{w}=2$ vortex mask. The solid curve corresponds to the Airy function (no mask). The dotted, dashed, and dot-dashed lines correspond to $N=2,4$, and 6 , respectively.

(shifting the fringe pattern projected on the sky directly correlates with modifying the POAM response). Interferometers are capable of limited POAM filtering with no additional hardware.

I use a Michelson beam combiner to illustrate the POAM behavior of interferometers, because it is the most common one used in astronomy. A Michelson interferometer could be used in conjunction with a rancorimeter for high-resolution measurements (Sect. 9). Another type of beam combiner, the Mach-Zehnder, has been used in laboratory experiments to measure POAM (Leach et al. 2002; Padgett et al. 2004). It can also be used for astronomical observations (Danchi et al. 2004). In this section, I form the DF, PSF, and POAM expansions of a Michelson interferometer and show that 1) the POAM response can be factored into interferometer and arm parts; 2) the POAM response is the sum of uncorrelated and correlated parts; 3) the POAM response is complicated by 

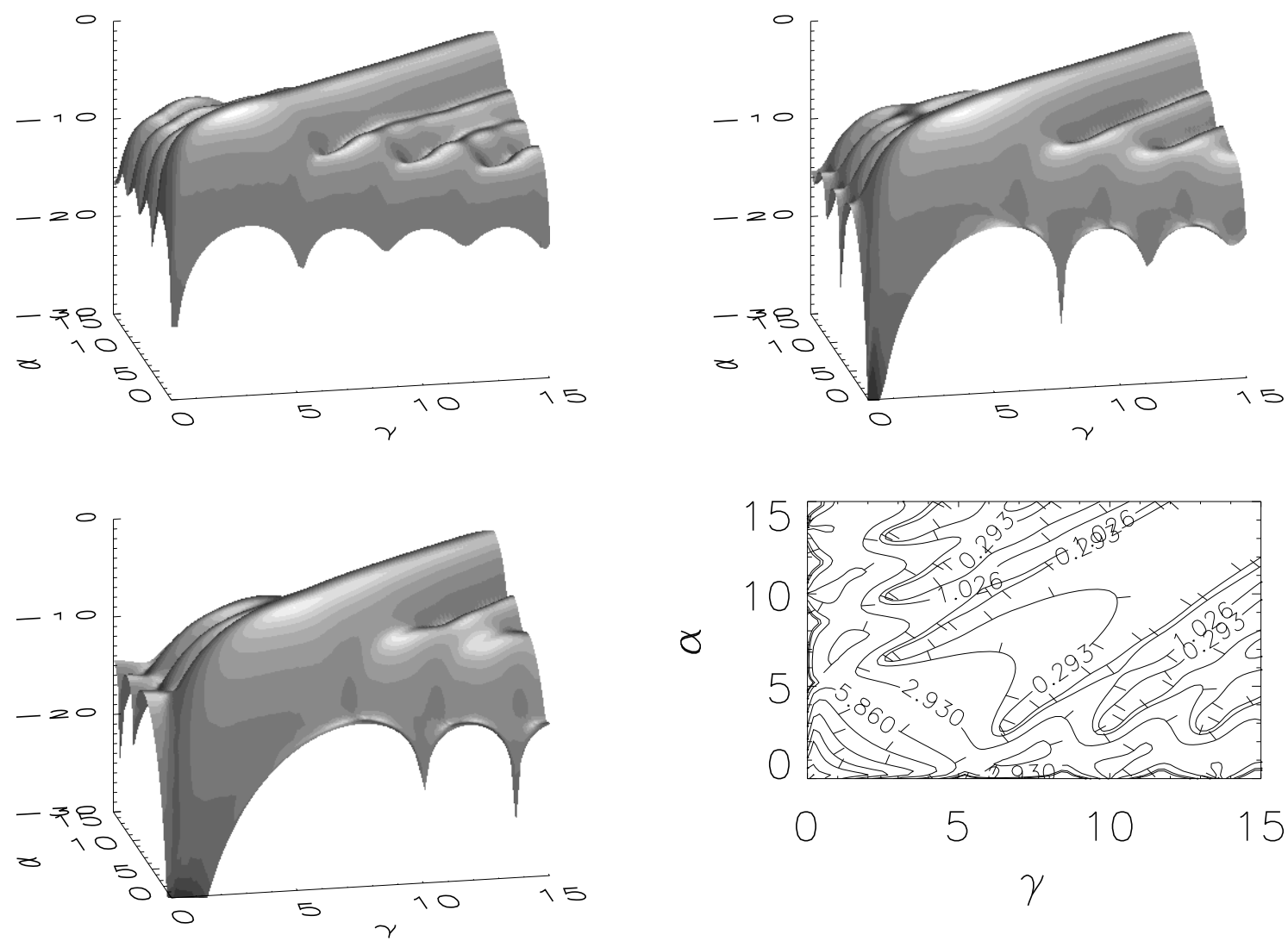

Fig. 8. The azimuthally averaged PSFs (in magnitudes) versus $\alpha=2 \pi \kappa R_{\mathrm{tel}} \rho$ and $\gamma=2 \pi \kappa R_{\mathrm{tel}} w / \Theta_{r}$ for a coronagraph using an $N=2$ (upper left), 4 (upper right), and 6 (lower left) alternating mask. The contour plot in the lower left contains the difference between the $N=2$ and $N=6$ masks.

aberrations in the arms; and 4) for unaberrated arms, the even states are passed at zero delay (standard mode) and odd states are passed at half a wavelength of delay (single-Bracewell mode).

\subsection{Classical forms}

A basic Michelson interferometer consists of two arms, $A$ and $B$. Each arm represents a path from a region in the observation plane (a telescope entrance pupil) to the image plane. The paths overlap at and after an exit pupil in the beam combiner. I modify Eq. (22b) to represent the propagation of the electric fields along the arms to the exit-pupil plane,

$E_{A}(\overrightarrow{\mathbf{B}}, \overrightarrow{\mathbf{\Omega}} ; \overrightarrow{\mathcal{R}}, t)=\mathcal{D}_{A}(\overrightarrow{\mathcal{R}}, \overrightarrow{\boldsymbol{\Omega}}) \mathrm{e}^{j 2 \pi \kappa\left(M \overrightarrow{\mathcal{R}}+\frac{1}{2} \overrightarrow{\mathbf{B}}\right) \cdot \overrightarrow{\mathbf{s}}_{x y}(\overrightarrow{\boldsymbol{\Omega}})} E(\overrightarrow{\boldsymbol{\Omega}} ; t)$

and

$E_{B}(\overrightarrow{\mathbf{B}}, \overrightarrow{\mathbf{\Omega}} ; \overrightarrow{\mathcal{R}}, t)=\mathcal{D}_{B}(\overrightarrow{\mathcal{R}}, \overrightarrow{\mathbf{\Omega}}) \mathrm{e}^{j 2 \pi \kappa\left(M \overrightarrow{\mathcal{R}}-\frac{1}{2} \overrightarrow{\mathbf{B}}\right) \cdot \overrightarrow{\mathbf{s}}_{x y}(\overrightarrow{\boldsymbol{\Omega}})} E(\overrightarrow{\mathbf{\Omega}} ; t)$,

where $\overrightarrow{\mathbf{B}}=\left(B_{x}, B_{y}\right)=(B \cos \beta, B \sin \beta)$ is the baseline vector between the centers of the two telescope entrance pupils in the observation plane, $B_{x}$ and $B_{y}$ are the Cartesian coordinates, $B$ is the baseline length, $\beta$ is the baseline azimuthal angle, and $\mathcal{D}_{A}(\overrightarrow{\mathcal{R}}, \overrightarrow{\mathbf{\Omega}})$ and $\mathcal{D}_{B}(\overrightarrow{\mathcal{R}}, \overrightarrow{\boldsymbol{\Omega}})$ are the amplitude/phase aberration functions of each arm projected onto the exit-pupil plane in the beam combiner. Assuming that the delay difference between the two arms is $d$, the electric field in the exit pupil of a single beam-combiner output is

$E(\overrightarrow{\mathbf{B}}, \overrightarrow{\mathbf{\Omega}} ; d, \overrightarrow{\mathcal{R}}, t)=E_{A}(\overrightarrow{\mathbf{B}}, \overrightarrow{\mathbf{\Omega}} ; \overrightarrow{\mathcal{R}}, t) \mathrm{e}^{-j 2 \pi \kappa \frac{1}{2} d}+E_{B}(\overrightarrow{\mathbf{B}}, \overrightarrow{\boldsymbol{\Omega}} ; \overrightarrow{\mathcal{R}}, t) \mathrm{e}^{j 2 \pi \kappa \frac{1}{2} d}$,

which is the sum of the arm electric fields delayed with respect to each other. I allocated half of the delay to each arm, but this choice is for mathematical convenience and completely arbitrary.

As in Eq. (23a), the image-plane electric field of the interferometer is the Fourier transform of the exit-pupil electric field

$E(\overrightarrow{\mathbf{B}}, \overrightarrow{\boldsymbol{\Omega}} ; d, \overrightarrow{\mathbf{r}}, t)=\frac{1}{A_{\mathrm{ep}}} \int \mathrm{d}^{2} \mathcal{R} \mathrm{e}^{-j 2 \pi \kappa \frac{\overrightarrow{\mathrm{r}}}{f} \cdot \overrightarrow{\mathcal{R}}} E(\overrightarrow{\mathbf{B}}, \overrightarrow{\boldsymbol{\Omega}} ; d, \overrightarrow{\mathcal{R}}, t)=D(\overrightarrow{\mathbf{B}}, \overrightarrow{\boldsymbol{\Omega}} ; d, \overrightarrow{\mathbf{r}}) E(\overrightarrow{\boldsymbol{\Omega}} ; t)$,

where

$$
\begin{aligned}
D(\overrightarrow{\mathbf{B}}, \overrightarrow{\boldsymbol{\Omega}} ; d, \overrightarrow{\mathbf{r}})= & \mathrm{e}^{j 2 \pi \kappa \frac{1}{2} \overrightarrow{\mathbf{B}} \cdot \overrightarrow{\mathbf{s}}_{x y}(\overrightarrow{\boldsymbol{\Omega}})} D_{A}(\overrightarrow{\mathbf{r}}, \overrightarrow{\boldsymbol{\Omega}}) \mathrm{e}^{-j 2 \pi \frac{1}{2} \kappa d}+\mathrm{e}^{-j 2 \pi \kappa \frac{1}{2} \overrightarrow{\mathbf{B}} \cdot \overrightarrow{\mathbf{s}}_{x y}(\overrightarrow{\boldsymbol{\Omega}})} D_{B}(\overrightarrow{\mathbf{r}}, \overrightarrow{\boldsymbol{\Omega}}) \mathrm{e}^{j 2 \pi \frac{1}{2} \kappa d} \\
& \approx \mathrm{e}^{j 2 \pi \kappa \frac{1}{2} B \rho \cos (\beta-\phi)} D_{A}(\overrightarrow{\mathbf{r}}, \overrightarrow{\boldsymbol{\Omega}}) \mathrm{e}^{-j 2 \pi \frac{1}{2} \kappa d}+\mathrm{e}^{-j 2 \pi \kappa \frac{1}{2} B \rho \cos (\beta-\phi)} D_{B}(\overrightarrow{\mathbf{r}}, \overrightarrow{\boldsymbol{\Omega}}) \mathrm{e}^{j 2 \pi \frac{1}{2} \kappa d}
\end{aligned}
$$



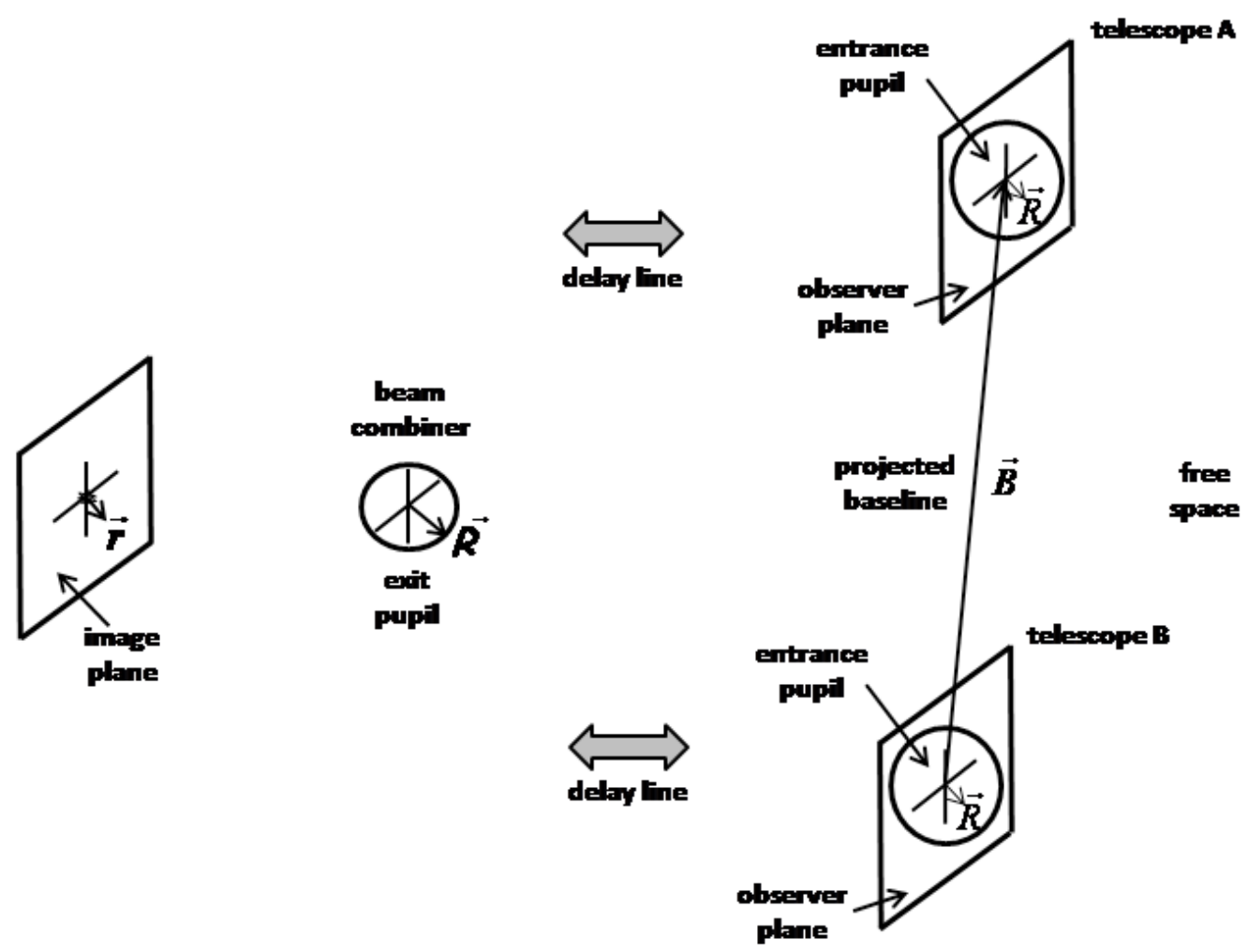

Fig. 9. An interferometer schematic diagram from a source on the celestial sphere to the image plane through two telescopes, two delay lines, and a beam combiner.

is the interferometric DF (IDF), and

$D_{A}(\overrightarrow{\mathbf{r}}, \overrightarrow{\boldsymbol{\Omega}})=\frac{1}{A_{\mathrm{ep}}} \int \mathrm{d}^{2} \mathcal{R} \mathrm{e}^{-j 2 \pi \kappa M\left[\frac{\overrightarrow{\mathbf{r}}}{\Theta}-\overrightarrow{\mathbf{s}}_{x y}(\overrightarrow{\boldsymbol{\Omega}})\right] \cdot \overrightarrow{\mathcal{R}}} \mathcal{D}_{A}(\overrightarrow{\mathcal{R}}, \overrightarrow{\boldsymbol{\Omega}})$

$D_{B}(\overrightarrow{\mathbf{r}}, \overrightarrow{\boldsymbol{\Omega}})=\frac{1}{A_{\text {ep }}} \int \mathrm{d}^{2} \mathcal{R} \mathrm{e}^{-j 2 \pi \kappa M\left[\frac{\overrightarrow{\mathbf{r}}}{\Theta}-\overrightarrow{\mathbf{s}}_{x y}(\overrightarrow{\boldsymbol{\Omega}})\right] \cdot \overrightarrow{\mathcal{R}}} \mathcal{D}_{B}(\overrightarrow{\mathcal{R}}, \overrightarrow{\boldsymbol{\Omega}})$

are the arm DFs (ADFs). The ADFs represent the electric-field impulse responses between the celestial sphere and the image plane for the arms. The IDF is the sum of delayed ADFs multipled by interferometric kernels. The second line of Eq. (46b) employs the small FOV approximation. The electric-field overlap integral becomes

$E(\overrightarrow{\mathbf{B}} ; d, \overrightarrow{\mathbf{r}}, t)=\int \mathrm{d}^{2} \Omega E(\overrightarrow{\mathbf{B}}, \overrightarrow{\mathbf{\Omega}} ; d, \overrightarrow{\mathbf{r}}, t)=\int \mathrm{d}^{2} \Omega D(\overrightarrow{\mathbf{B}}, \overrightarrow{\mathbf{\Omega}} ; d, \overrightarrow{\mathbf{r}}) E(\overrightarrow{\mathbf{\Omega}} ; t)$

The overlap integral, the IDF, and the electric field in the image plane have the same form as the generic versions of Sect. 4 ( $\overrightarrow{\mathbf{N}} \rightarrow$ $\overrightarrow{\mathbf{B}}$, and $\overrightarrow{\mathbf{a}} \rightarrow \overrightarrow{\mathbf{r}}, d$ ).

The intensity overlap integral, integrated over $\overrightarrow{\mathbf{r}}$ as well as $\overrightarrow{\boldsymbol{\Omega}}$, is

$$
\begin{aligned}
I(\overrightarrow{\mathbf{B}} ; d) & =\int \mathrm{d}^{2} r I(\overrightarrow{\mathbf{B}} ; d, \overrightarrow{\mathbf{r}})=\int \mathrm{d}^{2} r\left\langle\frac{1}{2}|E(\overrightarrow{\mathbf{B}} ; d, \overrightarrow{\mathbf{r}}, t)|^{2}\right\rangle=\int \mathrm{d}^{2} \Omega P(\overrightarrow{\mathbf{B}}, \overrightarrow{\mathbf{\Omega}} ; d) I(\overrightarrow{\mathbf{\Omega}}) \\
& =I_{0}+\operatorname{Re}\left\{I_{A B}(\overrightarrow{\mathbf{B}}) \mathrm{e}^{-j 2 \pi \kappa d}\right\}=I_{0}\left[1+\operatorname{Re}\left\{\mathcal{V}_{A B}(\overrightarrow{\mathbf{B}}) \mathrm{e}^{-j 2 \pi \kappa d}\right\}\right],
\end{aligned}
$$

where

$I_{0}=\int \mathrm{d}^{2} r I_{0}(\overrightarrow{\mathbf{r}})=\int \mathrm{d}^{2} \Omega P_{0}(\overrightarrow{\mathbf{\Omega}}) I(\overrightarrow{\mathbf{\Omega}})$

is the observed total zero-spacing flux,

$I_{A B}(\overrightarrow{\mathbf{B}})=\int \mathrm{d}^{2} r I_{A B}(\overrightarrow{\mathbf{B}} ; \overrightarrow{\mathbf{r}})=\int \mathrm{d}^{2} \Omega \mathrm{e}^{j 2 \pi \kappa B \rho \cos (\beta-\phi)} P_{A B}(\overrightarrow{\boldsymbol{\Omega}}) I(\overrightarrow{\boldsymbol{\Omega}})$ 
is the observed unnormalized visibility flux, $\mathcal{V}_{A B}(\overrightarrow{\mathbf{B}})$ is the observed normalized visibility,

$P(\overrightarrow{\mathbf{B}}, \overrightarrow{\boldsymbol{\Omega}} ; d)=\int \mathrm{d}^{2} r P(\overrightarrow{\mathbf{B}}, \overrightarrow{\boldsymbol{\Omega}} ; d, \overrightarrow{\mathbf{r}})=\int \mathrm{d}^{2} r|D(\overrightarrow{\mathbf{B}}, \overrightarrow{\boldsymbol{\Omega}} ; d, \overrightarrow{\mathbf{r}})|^{2}=P_{0}(\overrightarrow{\boldsymbol{\Omega}})+\operatorname{Re}\left\{\mathrm{e}^{j 2 \pi k B \rho \cos (\beta-\phi)} P_{A B}(\overrightarrow{\boldsymbol{\Omega}}) \mathrm{e}^{-j 2 \pi k d}\right\}$

is the interferometer PSF (IPSF),

$P_{0}(\overrightarrow{\boldsymbol{\Omega}})=\int \mathrm{d}^{2} r P_{0}(\overrightarrow{\mathbf{r}}, \overrightarrow{\boldsymbol{\Omega}})=\frac{1}{2}\left[P_{A A}(\overrightarrow{\mathbf{\Omega}})+P_{B B}(\overrightarrow{\mathbf{\Omega}})\right]$

is the mean PSF (MPSF) of the arms, the

$P_{A A}(\overrightarrow{\boldsymbol{\Omega}})=P_{0}(\overrightarrow{\boldsymbol{\Omega}})+\Delta P_{0}(\overrightarrow{\boldsymbol{\Omega}})=\int \mathrm{d}^{2} r P_{A A}(\overrightarrow{\mathbf{r}}, \overrightarrow{\boldsymbol{\Omega}})=\int \mathrm{d}^{2} r\left|D_{A}(\overrightarrow{\mathbf{r}}, \overrightarrow{\mathbf{\Omega}})\right|^{2}$

and

$P_{B B}(\overrightarrow{\boldsymbol{\Omega}})=P_{0}(\overrightarrow{\boldsymbol{\Omega}})-\Delta P_{0}(\overrightarrow{\boldsymbol{\Omega}})=\int \mathrm{d}^{2} r P_{B B}(\overrightarrow{\mathbf{r}}, \overrightarrow{\boldsymbol{\Omega}})=\int \mathrm{d}^{2} r\left|D_{B}(\overrightarrow{\mathbf{r}}, \overrightarrow{\boldsymbol{\Omega}})\right|^{2}$

are the arm PSFs (APSFs), and

$P_{A B}(\overrightarrow{\boldsymbol{\Omega}})=P_{B A}^{*}(\overrightarrow{\boldsymbol{\Omega}})=\int \mathrm{d}^{2} r P_{A B}(\overrightarrow{\mathbf{r}}, \overrightarrow{\boldsymbol{\Omega}})=\int \mathrm{d}^{2} r D_{A}(\overrightarrow{\mathbf{r}}, \overrightarrow{\boldsymbol{\Omega}}) D_{B}^{*}(\overrightarrow{\mathbf{r}}, \overrightarrow{\boldsymbol{\Omega}})$

is the cross PSF (CPSF). The unnormalized visibility contains recoverable information about the source intensity distribution on the celestial sphere. The zero-spacing flux, on the other hand, is of limited use, apart from normalization. If the arms are matched, or $\mathcal{D}_{A}(\overrightarrow{\mathcal{R}}, \overrightarrow{\boldsymbol{\Omega}})=\mathcal{D}_{B}(\overrightarrow{\mathcal{R}}, \overrightarrow{\boldsymbol{\Omega}}) \rightarrow \mathcal{D}(\overrightarrow{\mathcal{R}}, \overrightarrow{\boldsymbol{\Omega}})$ and $D_{A}(\overrightarrow{\mathbf{r}}, \overrightarrow{\boldsymbol{\Omega}})=D_{B}(\overrightarrow{\mathbf{r}}, \overrightarrow{\boldsymbol{\Omega}}) \rightarrow D(\overrightarrow{\mathbf{r}}, \overrightarrow{\boldsymbol{\Omega}})$, then the IDF becomes

$D(\overrightarrow{\mathbf{B}}, \overrightarrow{\mathbf{\Omega}} ; d, \overrightarrow{\mathbf{r}})=D(\overrightarrow{\mathbf{r}}, \overrightarrow{\mathbf{\Omega}}) \cos \left[2 \pi \kappa \frac{1}{2} B \rho \cos (\beta-\phi)-2 \pi \kappa d\right]$,

which is factored into the ADF and the interferometer response. Matched arms also lead to trivial auxilliary PSFs

$P_{A A}(\overrightarrow{\boldsymbol{\Omega}})=P_{B B}(\overrightarrow{\boldsymbol{\Omega}})=P_{0}(\overrightarrow{\boldsymbol{\Omega}})=P_{A B}(\overrightarrow{\boldsymbol{\Omega}}) \rightarrow P(\overrightarrow{\boldsymbol{\Omega}})=\int \mathrm{d}^{2} r|D(\overrightarrow{\mathbf{r}}, \overrightarrow{\boldsymbol{\Omega}})|^{2} \quad$ and $\quad \Delta P(\overrightarrow{\boldsymbol{\Omega}})=0$,

and a simple IPSF

$P(\overrightarrow{\mathbf{B}}, \overrightarrow{\boldsymbol{\Omega}} ; d)=\left[\int \mathrm{d}^{2} r|D(\overrightarrow{\mathbf{r}}, \overrightarrow{\boldsymbol{\Omega}})|^{2}\right] \cos ^{2}\left[2 \pi \kappa \frac{1}{2} B \rho \cos (\beta-\phi)-2 \pi \kappa d\right]=P(\overrightarrow{\boldsymbol{\Omega}}) \frac{1}{2}\{1+\cos [2 \pi \kappa B \rho \cos (\beta-\phi)-2 \pi \kappa d]\}$

which is factored into the APSF and the interferometer response.

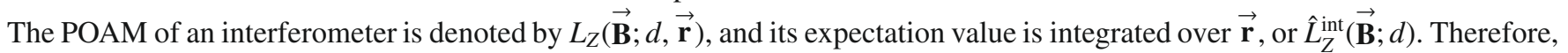
the POAM expectation value, as well as the integrated POAM probabilities, can be changed by varying the delay or moving the baseline vector. Single-Bracewell interferometers track $180^{\circ}$ from the central fringe, changing the observed POAM expectation value and inducing a null. If the arms are unaberrated and all visibilities are sampled up to baseline lengths of $B_{\max }$, the synthesized interferometer POAM expectation value should be the same as the POAM expectation value of a single unaberrated telescope of radius $\frac{1}{2} B_{\max }$.

\subsection{POAM expansions}

According to Table 1, the output states for a Michelson interferometer are

$E_{u}(B ; d, \overrightarrow{\mathbf{r}} ; t)=\sum_{m=-\infty}^{\infty} 2 \pi \int_{0}^{\infty} \mathrm{d} \rho \rho D_{u}^{-m}(B, \rho ; d, \overrightarrow{\mathbf{r}}) E_{m}(\rho ; t)$,

where the

$$
\begin{aligned}
D_{u}^{-m}(B, \rho ; d, \overrightarrow{\mathbf{r}}) & =\frac{1}{2 \pi} \int_{0}^{2 \pi} \mathrm{d} \phi \mathrm{e}^{j m \phi}\left[\frac{1}{2 \pi} \int_{0}^{2 \pi} \mathrm{d} \beta \mathrm{e}^{-j u \beta} D(\overrightarrow{\mathbf{B}}, \overrightarrow{\boldsymbol{\Omega}} ; d, \overrightarrow{\mathbf{r}})\right]=\frac{1}{2 \pi} \int_{0}^{2 \pi} \mathrm{d} \phi \mathrm{e}^{j m \phi} D_{u}(B, \overrightarrow{\boldsymbol{\Omega}} ; d, \overrightarrow{\mathbf{r}}) \\
& =\frac{1}{2 \pi} \int_{0}^{2 \pi} \mathrm{d} \phi \mathrm{e}^{j m \phi}\left[j^{u} J_{u}\left(2 \pi \kappa \frac{1}{2} B \rho\right) \mathrm{e}^{-j u \phi} D_{\operatorname{arm}}(\overrightarrow{\mathbf{r}}, \overrightarrow{\boldsymbol{\Omega}} ; d, u)\right]=j^{u} J_{u}\left(2 \pi \kappa \frac{1}{2} B \rho\right) D_{\text {arm }}^{-m+u}(\overrightarrow{\mathbf{r}}, \rho ; d, u)
\end{aligned}
$$

are the input/output gains of the IDF using the small FOV approximation (Appendix H), the

$$
\begin{aligned}
D_{\operatorname{arm}}(\overrightarrow{\mathbf{r}}, \overrightarrow{\boldsymbol{\Omega}} ; d, u) & =D_{A}(\overrightarrow{\mathbf{r}}, \overrightarrow{\boldsymbol{\Omega}}) \mathrm{e}^{-j 2 \pi \kappa \frac{1}{2} d}+(-1)^{u} D_{B}(\overrightarrow{\mathbf{r}}, \overrightarrow{\boldsymbol{\Omega}}) \mathrm{e}^{j 2 \pi \kappa \frac{1}{2} d} \\
& =E(u)\left[D_{A}(\overrightarrow{\mathbf{r}}, \overrightarrow{\boldsymbol{\Omega}}) \mathrm{e}^{-j 2 \pi \kappa \frac{1}{2} d}+D_{B}(\overrightarrow{\mathbf{r}}, \overrightarrow{\boldsymbol{\Omega}}) \mathrm{e}^{j 2 \pi \kappa \frac{1}{2} d}\right]+O(u)\left[D_{A}(\overrightarrow{\mathbf{r}}, \overrightarrow{\boldsymbol{\Omega}}) \mathrm{e}^{-j 2 \pi \kappa \frac{1}{2} d}-D_{B}(\overrightarrow{\mathbf{r}}, \overrightarrow{\boldsymbol{\Omega}}) \mathrm{e}^{j 2 \pi \kappa \frac{1}{2} d}\right]
\end{aligned}
$$


are the arm factors, the

$$
\begin{aligned}
D_{\mathrm{arm}}^{-m+u}(\overrightarrow{\mathbf{r}}, \rho ; d, u) & =\frac{1}{2 \pi} \int_{0}^{2 \pi} \mathrm{d} \phi \mathrm{e}^{j(m-u) \phi} D_{\mathrm{arm}}(\overrightarrow{\mathbf{r}}, \overrightarrow{\boldsymbol{\Omega}} ; d, u)=D_{A}^{-m+u}(\overrightarrow{\mathbf{r}}, \rho) \mathrm{e}^{-j 2 \pi \kappa \frac{1}{2} d}+(-1)^{u} D_{B}^{-m+u}(\overrightarrow{\mathbf{r}}, \rho) \mathrm{e}^{j 2 \pi \kappa \frac{1}{2} d} \\
& =E(u)\left[D_{A}^{-m+u}(\overrightarrow{\mathbf{r}}, \rho) \mathrm{e}^{-j 2 \pi \kappa \frac{1}{2} d}+D_{B}^{-m+u}(\overrightarrow{\mathbf{r}}, \rho) \mathrm{e}^{j 2 \pi \kappa \frac{1}{2} d}\right]+O(u)\left[D_{A}^{-m+u}(\overrightarrow{\mathbf{r}}, \rho) \mathrm{e}^{-j 2 \pi \kappa \frac{1}{2} d}-D_{B}^{-m+u}(\overrightarrow{\mathbf{r}}, \rho) \mathrm{e}^{j 2 \pi \kappa \frac{1}{2} d}\right](50)
\end{aligned}
$$

are the input sensitivities of the arm factors, the $D_{A}^{-m+u}(\overrightarrow{\mathbf{r}}, \rho)$ and $D_{B}^{-m+u}(\overrightarrow{\mathbf{r}}, \rho)$ are the input sensitivities of the ADFs, and

$E(u)=\frac{1}{2}\left[1+(-1)^{u}\right]$ and $O(u)=\frac{1}{2}\left[1-(-1)^{u}\right]$

are the even and odd selection functions. The arm factors are either sums or differences of the delayed ADFs, depending on the sign of the output state. If the arms are matched, then the source sensitivities of the arm factors become

$D_{\mathrm{arm}}^{-m+u}(\overrightarrow{\mathbf{r}}, \rho ; d, u)=2 D^{-m+u}(\overrightarrow{\mathbf{r}}, \overrightarrow{\mathbf{\Omega}})[E(u) \cos 2 \pi \kappa d-j O(u) \sin 2 \pi \kappa d]$.

The delay dependence is divided into even and odd POAM parts that are in quadrature with each other, which means that the intput/output gains can be changed by simply choosing a different delay tracking point.

According to Table 3, the output correlations for a Michelson interferometer are

$I_{u, v}(B ; d)=\int \mathrm{d}^{2} r I_{u, v}(B ; d, \overrightarrow{\mathbf{r}})=\sum_{m=-\infty}^{\infty} \sum_{n=\infty}^{\infty} 2 \pi \int_{0}^{\infty} \mathrm{d} \rho \rho \mathcal{P}_{u, v}^{-m+n}(B, \rho ; d) I_{m, n}(\rho)$,

where the

$$
\begin{aligned}
\mathcal{P}_{u, v}^{-m+n}(B, \rho ; d) & =\int \mathrm{d}^{2} r \mathcal{P}_{u, v}^{-m+n}(B, \rho ; d, \overrightarrow{\mathbf{r}})=\int \mathrm{d}^{2} r \frac{1}{2 \pi} \int_{0}^{2 \pi} \mathrm{d} \phi \mathrm{e}^{j(m-n) \phi} P_{u, v}(B, \overrightarrow{\mathbf{\Omega}} ; d, \overrightarrow{\mathbf{r}}) \\
& =\int \mathrm{d}^{2} r \frac{1}{2 \pi} \int_{0}^{2 \pi} \mathrm{d} \phi \mathrm{e}^{j(m-n) \phi} D_{u}(B, \overrightarrow{\mathbf{\Omega}} ; d, \overrightarrow{\mathbf{r}}) D_{v}^{*}(B, \overrightarrow{\mathbf{\Omega}} ; d, \overrightarrow{\mathbf{r}})=\int \mathrm{d}^{2} r \sum_{k=-\infty}^{\infty} D_{u}^{-k}(B, \rho ; d, \overrightarrow{\mathbf{r}}) D_{v}^{-k+m-n, *}(B, \rho ; d, \overrightarrow{\mathbf{r}}) \\
& =j^{u-v} J_{u}\left(2 \pi \kappa \frac{1}{2} B \rho\right) J_{v}\left(2 \pi \kappa \frac{1}{2} B \rho\right) \mathcal{P}_{\text {arm }}^{-m+n+u-v}(\rho ; d, u, v)
\end{aligned}
$$

are the input/output gains (combined \#2), the

$$
\begin{aligned}
\mathcal{P}_{\text {arm }}^{-m+n+u-v}(\rho ; d, u, v) & =\int \mathrm{d}^{2} r \mathcal{P}_{\text {arm }}^{-m+n+u-v}(\overrightarrow{\mathbf{r}}, \rho ; d, u, v)=\int \mathrm{d}^{2} r \frac{1}{2 \pi} \int_{0}^{2 \pi} \mathrm{d} \phi \mathrm{e}^{j(m-n-u+v) \phi} P_{\text {arm }}(\overrightarrow{\mathbf{r}}, \overrightarrow{\boldsymbol{\Omega}} ; d, u, v) \\
& =\int \mathrm{d}^{2} r \frac{1}{2 \pi} \int_{0}^{2 \pi} \mathrm{d} \phi \mathrm{e}^{j(m-n-u+v) \phi} D_{\text {arm }}(\overrightarrow{\mathbf{r}}, \overrightarrow{\boldsymbol{\Omega}} ; d, u) D_{\text {arm }}^{*}(\overrightarrow{\mathbf{r}}, \overrightarrow{\boldsymbol{\Omega}} ; d, v) \\
& =\mathcal{P}_{\text {uncorr }}^{-m+n+u-v}(\rho ; u-v)+(-1)^{v} \mathcal{P}_{\text {corr }}^{-m+n+u-v}(\rho ; d, u-v)
\end{aligned}
$$

are the input sensitivities of the arm factor product, the

$$
\begin{aligned}
\mathcal{P}_{\text {uncorr }}^{-m+n+u-v}(\rho ; u-v) & =\int \mathrm{d}^{2} r \mathcal{P}_{\text {uncorr }}^{-m+n+u-v}(\overrightarrow{\mathbf{r}}, \rho ; u-v)=\int \mathrm{d}^{2} r \frac{1}{2 \pi} \int_{0}^{2 \pi} \mathrm{d} \phi \mathrm{e}^{j(m-n-u+v) \phi} P_{\text {uncorr }}(\overrightarrow{\mathbf{r}}, \overrightarrow{\boldsymbol{\Omega}} ; u-v) \\
& =\frac{1}{2 \pi} \int_{0}^{2 \pi} \mathrm{d} \phi \mathrm{e}^{j(m-n-u+v) \phi}\left[2 E(u-v) P_{0}(\overrightarrow{\boldsymbol{\Omega}})+O(u-v) \Delta P_{0}(\overrightarrow{\mathbf{\Omega}})\right] \\
& =2 E(u-v) \mathcal{P}_{0}^{-m+n+u-v}(\rho)+O(u-v) \Delta \mathcal{P}_{0}^{-m+n+u-v}(\rho)
\end{aligned}
$$

are the zero-spacing input sensitivities (Appendix $\mathrm{H}$ ), and the

$$
\begin{aligned}
\mathcal{P}_{\text {corr }}^{-m+n+u-v}(\rho ; d, u-v) & =\int \mathrm{d}^{2} r \mathcal{P}_{\text {corr }}^{-m+n+u-v}(\overrightarrow{\mathbf{r}}, \rho ; d, u-v)=\int \mathrm{d}^{2} r \frac{1}{2 \pi} \int_{0}^{2 \pi} \mathrm{d} \phi \mathrm{e}^{j(m-n-u+v) \phi} P_{\text {corr }}(\overrightarrow{\mathbf{r}}, \overrightarrow{\boldsymbol{\Omega}} ; d, u-v) \\
& =\frac{1}{2 \pi} \int_{0}^{2 \pi} \mathrm{d} \phi \mathrm{e}^{j(m-n-u+v) \phi}\left[2 E(u-v) \operatorname{Re}\left\{P_{A B}(\overrightarrow{\mathbf{\Omega}}) \mathrm{e}^{-j 2 \pi \kappa d}\right\}+j 2 O(u-v) \operatorname{Im}\left\{P_{A B}(\overrightarrow{\mathbf{\Omega}}) \mathrm{e}^{-j 2 \pi \kappa d}\right\}\right] \\
& =2 E(u-v) \operatorname{Re}\left\{\mathcal{P}_{A B}^{-m+n+u-v}(\rho) \mathrm{e}^{-j 2 \pi \kappa d}\right\}+j 2 O(u-v) \operatorname{Im}\left\{\mathcal{P}_{A B}^{-m+n+u-v}(\rho) \mathrm{e}^{-j 2 \pi \kappa d}\right\}
\end{aligned}
$$

are the unnormalized visibility input sensitivities (Appendix H). Also according to Table 3, the output rancors are

$\mathcal{I}_{u}(B ; d)=\int \mathrm{d}^{2} r \mathcal{I}_{u}(B ; d, \overrightarrow{\mathbf{r}})=\sum_{k=-\infty}^{\infty} I_{k, k-u}(B ; d)=\sum_{m=-\infty}^{\infty} 2 \pi \int_{0}^{\infty} \mathrm{d} \rho \rho \mathcal{P}_{u}^{-m}(B, \rho ; d) \mathcal{I}_{m}(\rho)$. 
By the summing rule in Table 4, I obtain the input/output gains (combined \#1)

$\mathcal{P}_{u}^{-m}(B, \rho ; d)=\sum_{k=-\infty}^{\infty} \mathcal{P}_{k, k-u}^{-m}(B, \rho ; d)=\sum_{k=-\infty}^{\infty} j^{u} J_{k}\left(2 \pi \kappa \frac{1}{2} B \rho\right) J_{k-u}\left(2 \pi \kappa \frac{1}{2} B \rho\right) \mathcal{P}_{\mathrm{arm}}^{-m+u}(\rho ; d, k, k-u)$,

where

$\mathcal{P}_{\text {arm }}^{-m+u}(\rho ; d, k, k-u)=\mathcal{P}_{\text {uncorr }}^{-m+u}(\rho ; u)+(-1)^{k-u} \mathcal{P}_{\text {corr }}^{-m+u}(\rho ; d, u)$,

$\mathcal{P}_{\text {uncorr }}^{-m+u}(\rho ; u)=2 E(u) \mathcal{P}_{0}^{-m+u}(\rho)+O(u) \Delta \mathcal{P}_{0}^{-m+u}(\rho)$

and

$\mathcal{P}_{\text {corr }}^{-m+u}(\rho ; d, u)=2 E(u) \operatorname{Re}\left\{\mathcal{P}_{A B}^{-m+u}(\rho) \mathrm{e}^{-j 2 \pi \kappa d}\right\}+j 2 O(u) \operatorname{Im}\left\{\mathcal{P}_{A B}^{-m+u}(\rho) \mathrm{e}^{-j 2 \pi \kappa d}\right\}$.

The zero-spacing part of the correlations and rancors has been expanded into POAM components, but ultimately the sum $I_{0}$ will be of interest only for normalization. If the arms are perfectly matched, only the zero-spacing components with even indices will be non-zero. Also, even (odd) indices determine whether the real (imaginary) part of the unnormalized visibility components are non-zero.

If the arms are matched, the input/output gains (combined \#2 and combined \#1) become

$\mathcal{P}_{u, v}^{-m+n}(B, \rho ; d)=2 j^{u-v} J_{u}\left(2 \pi \kappa \frac{1}{2} B \rho\right) J_{v}\left(2 \pi \kappa \frac{1}{2} B \rho\right) \mathcal{P}^{-m+n+u-v}(\rho)\left\{E(u-v)+(-1)^{v}[E(u-v) \cos 2 \pi \kappa d-j O(u-v) \sin 2 \pi \kappa d]\right\}(54 \mathrm{a})$

and

$\mathcal{P}_{u}^{-m}(B, \rho ; d)=2 \mathcal{P}^{-m+u}(\rho)\left\{\delta_{u, 0}+j^{u} J_{u}(2 \pi \kappa B \rho)[E(u) \cos 2 \pi \kappa d-j O(u) \sin 2 \pi \kappa d]\right\}$.

Inside the curly brackets of both equations, the first term represents the uncorrelated response, while the others represent the correlated response. How the delay dependence changes with indices $u-v$ and $u$ is clearly visible. I take this analysis one step further by assuming that the arms are unaberrated and the telescope FOVs are much larger than the interferometer's, which means that $P(\overrightarrow{\mathbf{\Omega}}) \rightarrow C=$ constant (Appendix F), $\mathcal{P}^{-m+n+u-v}(\rho) \rightarrow C \delta_{n, m-u+v}$ and $\mathcal{P}^{-m+u}(\rho) \rightarrow C \delta_{m, u}$. Therefore, the gains further simplify to

$\mathcal{P}_{u, v}^{-m+n}(B, \rho ; d)=2 C j^{u-v} J_{u}\left(2 \pi \kappa \frac{1}{2} B \rho\right) J_{v}\left(2 \pi \kappa \frac{1}{2} B \rho\right) \delta_{n, m-u+v}\left\{E(u-v)+(-1)^{v}[E(u-v) \cos 2 \pi \kappa d-j O(u-v) \sin 2 \pi \kappa d]\right\}$

and

$\mathcal{P}_{u}^{-m}(B, \rho ; d)=2 C \delta_{m, u}\left\{\delta_{u, 0}+j^{u} J_{u}(2 \pi \kappa B \rho)[E(u) \cos 2 \pi \kappa d-j O(u) \sin 2 \pi \kappa d]\right\}$,

making the important dependencies even clearer. Note that each output correlation (rancor) arises from only a single input correlation (rancor) because of the Kronecker deltas in the input/output gains of the ADFs.

Changing the delay tracking point changes the forms of the gains. If $d=0$, then only the $u, v(u)=$ even correlation (rancor) gains are non-zero. Conversely, if the delay lines are offset tracking at $d=0.5 / \kappa$, then only the $u, v(u)=$ odd correlation (rancor) gains are non-zero. Single-Bracewell nulling interferometers take advantage of this behavior, which is equivalent to shifting the position of the fringes projected on the sky, to null the central star (Bracewell 1986). To perform high-resolution observations, an interferometer can be used as the front end of a rancorimeter (Sect. 9).

Last, I briefly discuss POAM and its possible relationship to astronomical imaging. A fringe packet, obtained when slewing the delay line, contains entangled spectral and spatial information. Multiple slews across fringe packets using different baseline lengths and orientations leads to unnormalized visibility cubes that can be transformed into image cubes (Elias et al. 2007). POAM from the celestial sphere contains spatial information versus wavenumber, and I have shown that unnormalized visibilities can be expanded into correlations and rancors. It follows that fringe packets contain entangled spectral and POAM information. In principle, rancorimetric and interferometric data could be used as inputs to new image-processing algorithms. Whether this idea has merit is a subject for future research.

\section{POAM propagation through limited rancorimeter}

Rancorimetry is the process of measuring correlations. An instrument that measures these correlations is called a rancorimeter. One possible rancorimeter is the Mach-Zehnder interferometer employed as a POAM sorter (Leach et al. 2002). In this paper, however, I describe a limited rancorimeter, which observes only rancors (infinite sums of correlations). In other words, only sources that exhibit azimuthal dependence about the FOV center register non-zero rancors. Although limited rancorimeters do not produce complete source POAM descriptions, they tend to be simpler to build and operate. In this section, I convert a coronagraph (Sect. 7) into a limited rancorimeter by placing a rotating opaque wedge mask in the TIP. Also, I provide an example of super-Rayleigh modeling of unresolved binary stars. 


\subsection{Coronagraph with a rotating wedge mask}

A limited rancorimeter can be constructed from an existing coronagaph (Sect. 7) by replacing stationary coronagraphic masks with a rotating wedge mask, which is a circular opaque disk with a clear sector up to $180^{\circ}$ in size. It can measure only rancors, which are infinite sums of correlations. Different wedge sizes can be used to optimize the range of input/output gains on the celestial sphere, if desired. Padgett et al. (2004) have used a rotating wedge to demonstrate the POAM version of the Uncertainty Principle. The rotating wedge mask is strikingly analogous to a rotating polarizer that measures PSAM (modulation contains information).

An ideal wedge mask has unity throughput within the open region and is independent of position on celestial sphere, which leads to the functional form

$M(\overrightarrow{\mathbf{r}}, \overrightarrow{\mathbf{\Omega}}) \rightarrow M(\xi ; N, \delta)=p\left(\xi-\delta, \frac{2 \pi}{N}\right)$,

where $2 \pi / N$ is the opening angle of the wedge, $N=1,2,3, \ldots$ is the wedge integer, and $\delta$ is the wedge rotation angle. The Fourier components of this wedge are

$M_{q}(r, \overrightarrow{\boldsymbol{\Omega}}) \rightarrow M_{q}(N, \delta)=\frac{1}{2 \pi} \int_{0}^{2 \pi} \mathrm{d} \xi \mathrm{e}^{-j q \xi} M(\xi ; N, \delta)=\left[\frac{1}{N} \operatorname{sinc}\left(\frac{q}{N} \pi\right)\right] \mathrm{e}^{-j q \delta}=f_{N}(q) \mathrm{e}^{-j q \delta}$,

where $f_{N}(q)$ is the POAM selection function. Substituting this wedge mask into the unaberrated CDF of Eq. (35), I obtain the CDF (now rancorimeter) input/output gains

$D_{u}^{-m}(w, \rho)=\sum_{k=-\infty}^{\infty} \sum_{p=-\infty}^{\infty}\left[2 \pi \int_{0}^{r_{\mathrm{tip}}} \mathrm{d} r r D_{u}^{-k}(w, r) D_{p}^{-m}(r, \rho) f_{N}(k-p)\right] \mathrm{e}^{-j(k-p) \delta}=\sum_{k=-\infty}^{\infty} \sum_{p=-\infty}^{\infty} \mathcal{D}_{u, p}^{-k,-m}(w, \rho ; N) \mathrm{e}^{-j(k-p) \delta}$.

I assumed that the reimaging system does not depend on celestial sphere position. According to Table 4, the CPSF input/output gains (combined \#1) can be determined directly from the CDF input/output gains

$$
\begin{aligned}
\mathcal{P}_{u}^{-m}(w, \rho) & =\sum_{g=-\infty}^{\infty} \sum_{h=-\infty}^{\infty} D_{h}^{-g}(w, \rho) D_{h-u}^{-g+m, *}(w, \rho) \\
& =\sum_{k=-\infty}^{\infty} \sum_{l=-\infty}^{\infty} \sum_{p=-\infty}^{\infty} \sum_{q=-\infty}^{\infty}\left[\sum_{g=-\infty}^{\infty} \sum_{h=-\infty}^{\infty} \mathcal{D}_{h, p}^{-k,-g}(w, \rho ; N) \mathcal{D}_{h-n, q}^{-l,-g+m, *}(w, \rho ; N)\right] \mathrm{e}^{-j(k-l-p+q) \delta} .
\end{aligned}
$$

The multiple sums arise from the wedge modulation. For an unaberrated instrument, the CDF and CPSF input/output gains become

$D_{u}^{-m}(w, \rho)=\mathcal{D}_{u, m}^{-u,-m}(w, \rho ; N) \mathrm{e}^{-j(u-m) \delta}$

and

$\mathcal{P}_{u}^{-m}(w, \rho)=\left[\sum_{g=-\infty}^{\infty} \sum_{h=-\infty}^{\infty} \mathcal{D}_{h, g}^{-h,-g}(w, \rho ; N) \mathcal{D}_{h-u, g-m}^{-h+u,-g+m}(w, \rho ; N)\right] \mathrm{e}^{-j(u-m) \delta}=\mathcal{P}(w, \rho ; u, m ; N) \mathrm{e}^{-j(u-m) \delta}$.

Many of the sums disappear because the TDF and RDF of unaberrated instruments have Kronecker deltas between indices. The complex conjugation disappears because $\mathcal{D}_{h-u, q-m}^{-h+u,-g+m}(w, \rho ; N)$ is real for an unaberrated telescope. Also, $\mathcal{P}(w, \rho ; \pm u, \pm m ; N)=$ $\mathcal{P}(w, \rho, u, m ; N)$. I plot the lowest $u=m$ intensity input/output gains (combined \#1) in Figs. 10 and 11 for $N=2,3$, respectively. The $u=m=0$ gains exhibit a peak at the center, while the others exhibit a null.

To discuss measurements of the source rancors from the reimage-plane rancors, I begin with the rancored/rancored entry from Table 3,

$$
\begin{aligned}
I(\overrightarrow{\mathbf{w}}) & =\sum_{u=-\infty}^{\infty} \mathcal{I}_{u}(w) \mathrm{e}^{j u \omega} \\
& =\sum_{u=-\infty}^{\infty}\left[\sum_{m=-\infty}^{\infty} 2 \pi \int_{0}^{\infty} \mathrm{d} \rho \rho \mathcal{P}_{u}^{-m}(w, \rho) \mathcal{I}_{m}(\rho)\right] \mathrm{e}^{j u \omega}=\sum_{u=-\infty}^{\infty}\left[\sum_{m=-\infty}^{\infty} 2 \pi \int_{0}^{\infty} \mathrm{d} d \rho \rho \mathcal{P}(w, \rho ; u, m ; N) \mathrm{e}^{-j(u-m) \delta} \mathcal{I}_{m}(\rho)\right] \mathrm{e}^{j u \omega} .
\end{aligned}
$$

There are several ways to proceed with the analysis. I have chosen the most basic.

For now, consider only a ring source at $\rho=\rho_{*}$. Also, assume that the reimage plane contains only a single detector that captures most of the light incident upon it (analogous to a classical PSAM-measuring instrument). Eq. (59) now becomes

$$
\begin{aligned}
\int \mathrm{d}^{2} w I(\overrightarrow{\mathbf{w}}) & =2 \pi \int_{0}^{\infty} \mathrm{d} w w \mathcal{I}_{0}(w) \rightarrow \mathcal{I}_{0}^{\mathrm{rip}}(N, \delta) \\
& \approx \mathcal{P}(0 ; N) \mathcal{I}_{0}^{\mathrm{cs}}+\sum_{m=1}^{N_{m}} \mathcal{P}(m ; N) \operatorname{Re}\left\{\mathcal{I}_{m}^{\mathrm{cs}}\right\} \cos m \delta-\sum_{m=1}^{N_{m}} \mathcal{P}(m ; N) \operatorname{Im}\left\{\mathcal{I}_{m}^{\mathrm{cs}}\right\} \sin m \delta
\end{aligned}
$$



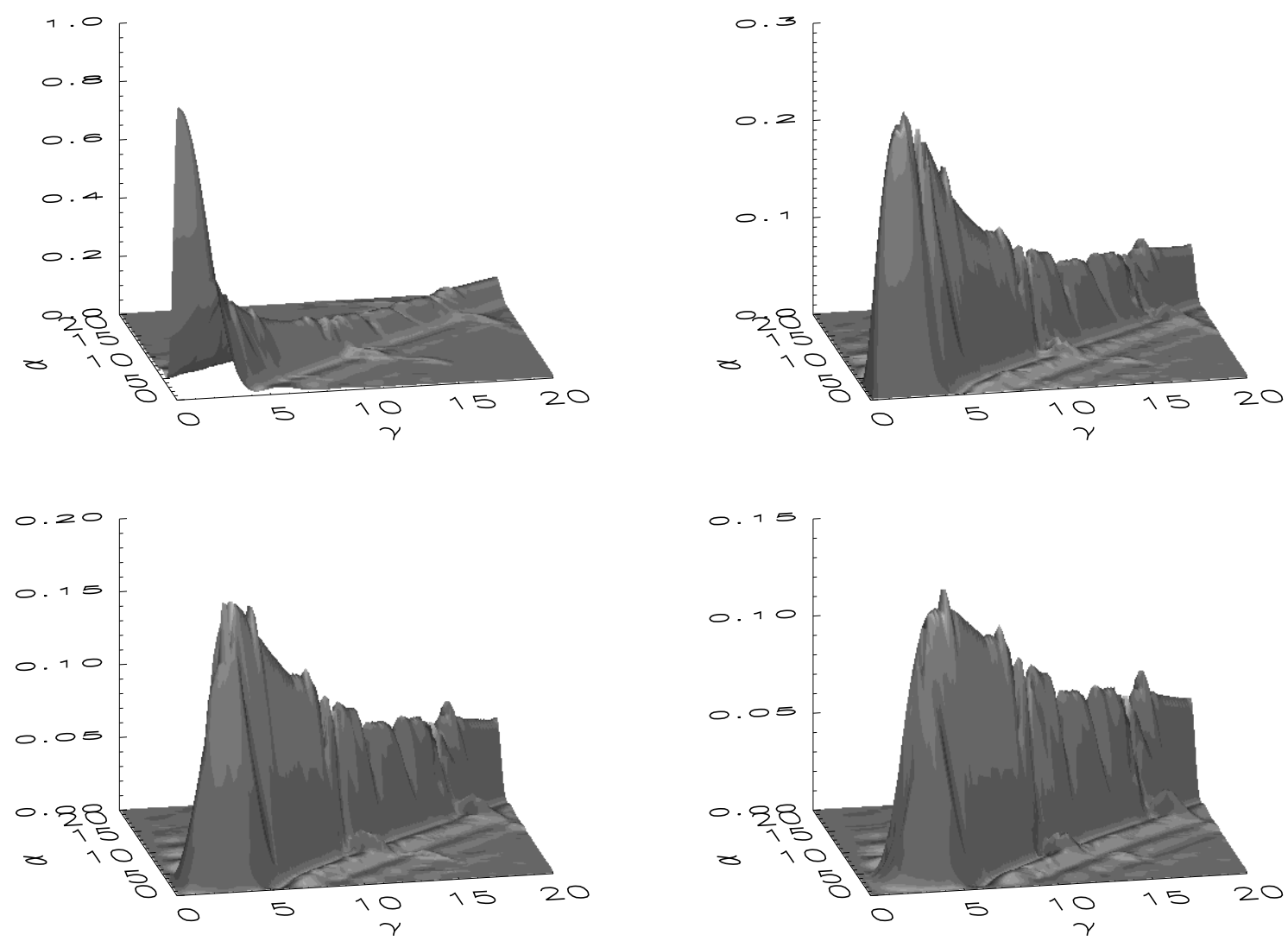

Fig. 10. The $N=2$ wedge mask rancorimeter input/output gains $u=m=0,1,2,3$ (upper left, upper right, lower left, lower right) for $\alpha=2 \pi \kappa R_{\mathrm{tel}} \rho$ and $\gamma=2 \pi \kappa R_{\text {tel }} w / \Theta_{r}$.
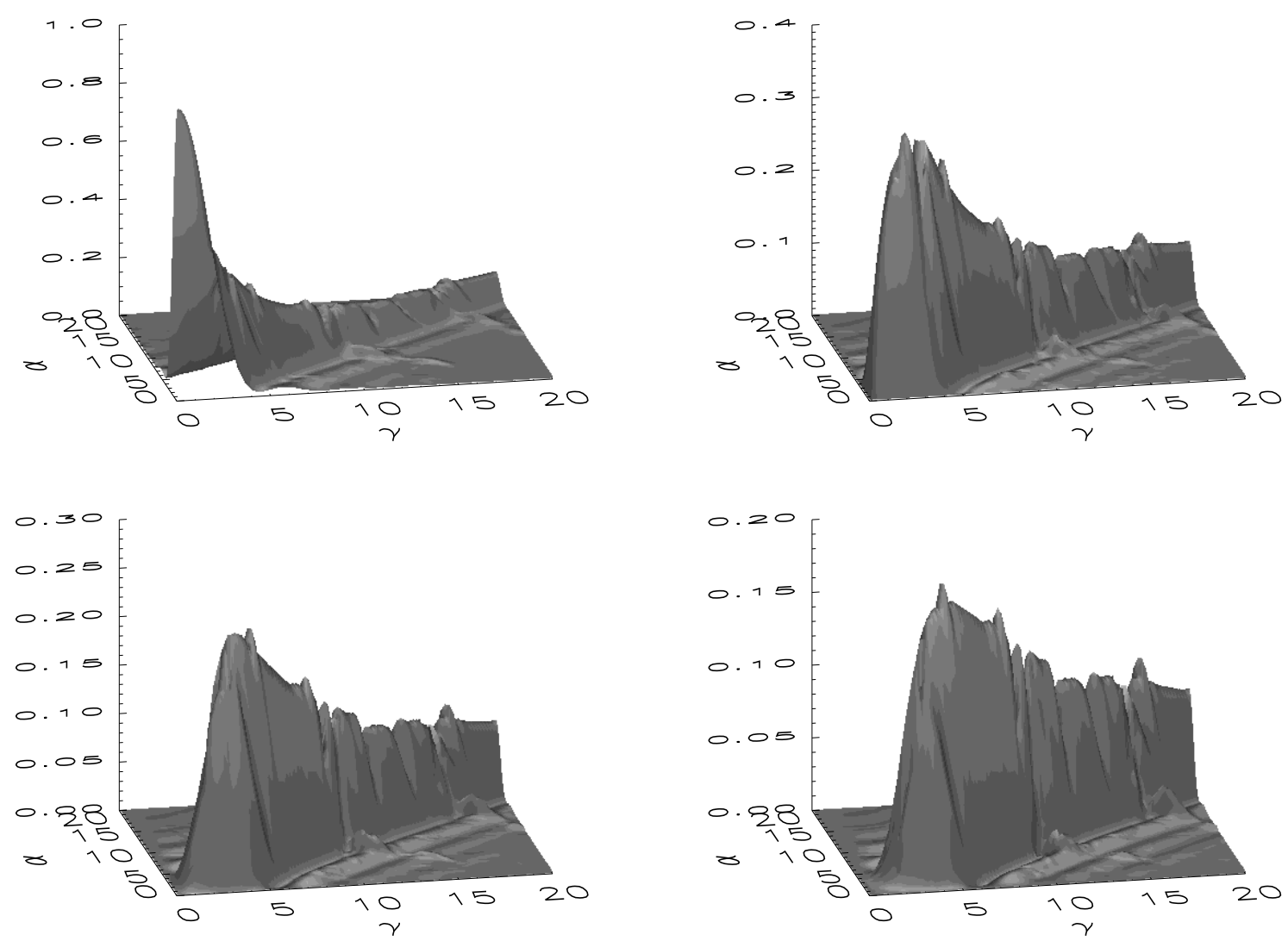

Fig. 11. The $N=3$ wedge mask rancorimeter input/output gains $u=m=0,1,2,3$ (upper left, upper right, lower left, lower right) for $\alpha=2 \pi \kappa R_{\mathrm{tel}} \rho$ and $\gamma=2 \pi \kappa R_{\mathrm{tel}} w / \Theta_{r}$. 
where $N_{m}$ is the number of expected source rancors, the $\mathcal{I}_{m}^{\mathrm{cs}} \approx \mathcal{I}_{m}\left(\rho_{*}\right)$, and the

$\mathcal{P}\left(\rho_{*} ; m ; N\right)=\mathcal{P}(m ; N)=2 \pi \int_{0}^{\infty} \mathrm{d} w w \mathcal{P}\left(w, \rho_{*} ; 0, m ; N\right)$

are the integrated gains. Only the unrancored term remains in the reimage plane. Since $\mathcal{I}_{m}^{\mathrm{cs}}=\mathcal{I}_{-m}^{c s, *}$, the $\pm m$ terms are added together to form separate real and imaginary parts. Equation (60a) has effectively become a Fourier series in $\delta$, which means that the $\mathcal{I}_{m}^{\mathrm{cs}}$ can be estimated directly if the $\mathcal{P}(m ; N)$ are known a priori.

If the wedge is rotated to $N_{\delta}$ discrete azimuthal angles $\delta \rightarrow \delta_{i}$ in the course of a single limited rancorimetry observation, then Eq. (60a) may be rewritten in matrix form

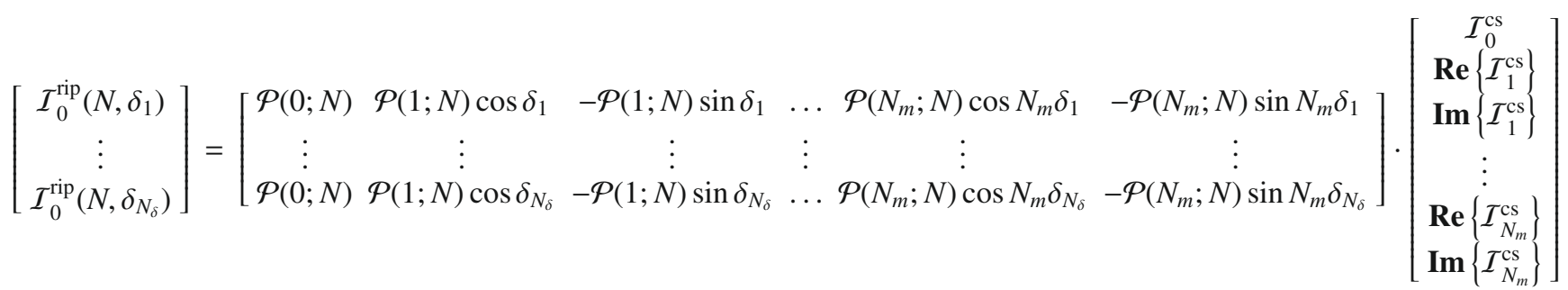

or

$\overrightarrow{\mathcal{I}}_{0}^{\text {rip }}(N)=\stackrel{\mathrm{E}}{N}_{N} \cdot \overrightarrow{\mathcal{I}}^{\mathrm{cs}}$

The least-squares inversion is

$\overrightarrow{\mathcal{I}}^{\mathrm{cs}}=\left[\stackrel{\leftrightarrow}{\mathbf{E}}_{N}^{T} \cdot \stackrel{\leftrightarrow}{\mathbf{E}}_{N}\right]^{-1} \cdot \stackrel{\leftrightarrow}{\mathbf{E}}_{N}^{T} \cdot \overrightarrow{\mathcal{I}}_{0}^{\mathrm{rip}}(N)$

The condition $N_{\delta}>N_{m}+1$ must be met for a non-degenerate solution. If the (co)variances of the $\mathcal{I}_{0}^{\text {rip }}\left(N, \delta_{i}\right)$ are known, then weights can be applied to the least-squares solution

$\overrightarrow{\mathcal{I}}^{\mathrm{cs}}=\left[\stackrel{\leftrightarrow}{\mathbf{E}}_{N}^{T} \cdot \stackrel{\leftrightarrow}{\mathbf{C}}^{-1} \cdot \stackrel{\leftrightarrow}{\mathbf{E}}_{N}\right]^{-1} \cdot \stackrel{\leftrightarrow}{\mathbf{E}}_{N}^{T} \cdot \stackrel{\leftrightarrow}{\mathbf{C}}^{-1} \cdot \overrightarrow{\mathcal{I}}_{0}^{\mathrm{rip}}(N)$

where $\stackrel{\leftrightarrow}{\mathbf{C}}^{-1}$ is the covariance matrix of $\overrightarrow{\mathcal{I}}_{0}^{\text {rip }}(N)$. Since only a finite number of $\mathcal{P}(m ; N)$ will be significant for a given wedge number $N$, the chosen $m$ will likely not be continuous between 0 and $N_{m}$. Systems of equations using different $N$ can be combined for a wider range of observed source rancors, but they must be normalized properly. Complicated astronomical scenes containing multiple non-zero rings must be deconvolved from the input/output gains and $\rho$ integrals. Most of the time, a detector array in the image plane is required to perform this task, which is beyond the scope of this paper.

\subsection{Super-Rayleigh modeling of unresolved binary stars}

Tamburini et al. (2006) used optical vortices in conjunction with LG modes to overcome the classical Rayleigh imaging resolution limit in the laboratory. They also mentioned the possibility of astronomical applications. In this section, I demonstrate a similar concept, super-Rayleigh modeling. The limited rancorimeter (Sect. 9.1) modulates rancors from the celestial sphere, leading to indirect measurements of the separation and orientation of stars in an unresolved binary system.

I assume that the stars are point sources and that the telescope always tracks the center of light (COL) of the binary system, which means that

$I(\overrightarrow{\boldsymbol{\Omega}})=I_{1} \frac{\delta\left(\rho-\rho_{1, *}\right)}{\rho} \delta\left(\phi-\phi_{1, *}\right)+I_{2} \frac{\delta\left(\rho-\rho_{2, *}\right)}{\rho} \delta\left(\phi-\phi_{2, *}\right)=I_{1}\left[\frac{\delta\left(\rho-\rho_{1, *}\right)}{\rho} \delta\left(\phi-\phi_{*}\right)+q_{L} \frac{\delta\left(\rho-\rho_{2, *}\right)}{\rho} \delta\left(\phi-\phi_{*}-180^{\circ}\right)\right]$,

where $I_{1}$ and $I_{2}$ are the stellar brightnesses, $q_{L}=I_{2} / I_{1}$ is the stellar light ratio, $\rho_{*}=\rho_{1, *}+\rho_{2, *}$ is the total stellar separation, $\rho_{1, *}$ and $\rho_{2, *}$ are the COL separations, and $\phi_{1, *}=\phi_{*}$ and $\phi_{2, *}=\phi_{*}+180^{\circ}$ are the azimuthal angles. These quantities are defined further in Appendix I. The corresponding rancors are

$\mathcal{I}_{m}(\rho)=\frac{I_{1}}{2 \pi} \frac{\delta\left(\rho-\rho_{1, *}\right)}{\rho} \mathrm{e}^{j m \phi_{*}}-\frac{I_{2}}{2 \pi} \frac{\delta\left(\rho-\rho_{2, *}\right)}{\rho} \mathrm{e}^{j m \phi_{*}}$.

Substituting this equation into Eq. (60a), I obtain

$$
\begin{aligned}
\mathcal{I}_{0}^{\mathrm{rip}}(N, \delta)= & i_{0}+\sum_{m=1}^{N_{m}} i_{c, m} \cos m \delta+\sum_{m=1}^{N_{m}} i_{s, m} \sin m \delta \\
= & I_{1}\left[\mathcal{P}\left(\rho_{1} ; 0 ; N\right)+q_{L} \mathcal{P}\left(\rho_{2} ; 0 ; N\right)\right]+\sum_{m=1}^{N_{m}} 2 I_{1}\left[\mathcal{P}\left(\rho_{1} ; m ; N\right)-q_{L} \mathcal{P}\left(\rho_{2} ; m ; N\right)\right] \cos m \phi_{*} \cos m \delta \\
& +\sum_{m=1}^{N_{m}} 2 I_{1}\left[\mathcal{P}\left(\rho_{1} ; m ; N\right)-q_{L} \mathcal{P}\left(\rho_{2} ; m ; N\right)\right] \sin m \phi_{*} \sin m \delta
\end{aligned}
$$


Observations over multiple $\delta$ can be used to obtain the coefficients via least squares (Sect. 9.1). The binary azimuthal angle can then be obtained redundantly using the ratios

$\phi_{*}=\tan ^{-1} \frac{i_{s, m}}{i_{c, m}} \quad(m \geq 1)$

Further, the ratios

$\frac{i_{m}}{i_{0}}=\frac{\sqrt{i_{c, m}^{2}+i_{s, m}^{2}}}{i_{0}}=2 \frac{\mathcal{P}\left(\rho_{1, *} ; m ; N\right)-q_{L} \mathcal{P}\left(\rho_{2, *} ; m ; N\right)}{\mathcal{P}\left(\rho_{1, *} ; 0 ; N\right)+q_{L} \mathcal{P}\left(\rho_{2, *} ; 0 ; N\right)} \quad(m \geq 1)$

can be used to solve for $\rho_{1, *}, \rho_{2, *}$, and $q_{L}$. For an unresolved binary star, the $i_{m} / i_{0}$ ratios will be relatively small, on the order of $10^{-2 \text { to }-3}$, comparable to normalized interferometric visibility measurements of stellar diameters near the zero and normalized polarimetric measurements of Be stars and red supergiants. The smallest observable ratio will determine the ultimate resolution. To measure such small values, calibration of instrumental POAM (optical aberrations and atmospheric turbulence; Sect. 11) is vital. So, is this type of instrument feasible?

Extreme Adaptive Optics (EAO) systems greatly minimize the effects of optical and atmospheric aberrations. When employed with coronagraphs, they can be used to detect extrasolar planets (Boccaletti et al. 2002; Crepp et al. 2007). One or more space-based optical coronagraph missions may be launched within the next 10-20 years (Guyon et al. 2006). Atmospheric turbulence is not an issue, plus the optics train will be the best ever built. To get the highest possible return on investment, other instrument packages, such as cameras and spectrographs, will likely be installed. There is no reason that a limited rancorimeter should not be included, to increase the spatial resolution of the telescope.

\section{Classes of astronomical POAM}

Harwit (2003) listed several examples of exotic emission mechanisms for astronomical POAM. Typical objects, such as $\approx$ unresolved spherical stars, generate no POAM because propagation smooths out the random amplitudes and phases of the electric fields. "Natural light" sources are useful for calibration. In this section, I classify the types of non-zero POAM that arise from the celestial sphere: intrinsic, structure, and pointing.

Consider a source that consists only of a single vortex state $k$ at the FOV center (Sect. 2.2),

$E(\overrightarrow{\mathbf{\Omega}} ; t)=\mathcal{E}(\rho ; t) \mathrm{e}^{j k \phi}$.

The intensity is independent of $\phi$,

$I(\overrightarrow{\mathbf{\Omega}})=\left\langle\frac{1}{2}|\mathcal{E}(\rho ; t)|^{2}\right\rangle=\mathcal{I}(\rho)$.

Not surprisingly, there is only a single state in its spectrum

$E_{m}(\rho ; t)=\mathcal{E}(\rho ; t) \delta_{m, k}$,

which yields a single identical component in the correlation and rancor spectra,

$I_{m, n}(\rho)=\left\langle\frac{1}{2}|\mathcal{E}(\rho ; t)|^{2}\right\rangle \delta_{m, k} \delta_{n, k}=\mathcal{I}(\rho) \delta_{m, k} \delta_{n, k}$

and

$\mathcal{I}_{m}(\rho)=\left\langle\frac{1}{2}|\mathcal{E}(\rho ; t)|^{2}\right\rangle \delta_{m, 0}=\mathcal{I}(\rho) \delta_{m, 0}$

Sources containing only a single vortex exhibit intrinsic POAM. They are unrancored, which results from their lack of $\phi$-dependence.

Now consider a source that consists of two vortex states $k$ and $l$ at the FOV center (Sect. 3),

$E(\overrightarrow{\mathbf{\Omega}} ; t)=\mathcal{E}^{(1)}(\rho ; t) \mathrm{e}^{j k \phi}+\mathcal{E}^{(2)}(\rho ; t) \mathrm{e}^{j l \phi}$.

The corresponding intensity is

$$
\begin{aligned}
I(\overrightarrow{\boldsymbol{\Omega}}) & =\left\langle\frac{1}{2}\left|\mathcal{E}^{(1)}(\rho ; t)\right|^{2}\right\rangle+\left\langle\frac{1}{2}\left|\mathcal{E}^{(2)}(\rho ; t)\right|^{2}\right\rangle+2 \operatorname{Re}\left\{\left\langle\frac{1}{2} \mathcal{E}^{(1)}(\rho ; t) \mathcal{E}^{(2), *}(\rho ; t)\right\rangle \mathrm{e}^{j(k-l) \phi}\right\} \\
& =\mathcal{I}^{(1,1)}(\rho)+\mathcal{I}^{(2,2)}(\rho)+2 \operatorname{Re}\left\{\mathcal{I}^{(1,2)}(\rho) \mathrm{e}^{j(k-l) \phi}\right\},
\end{aligned}
$$

which exhibits a $\phi$ dependence when the states $k$ and $l$ are correlated. The state spectrum is

$E_{m}(\rho ; t)=\mathcal{E}^{(1)}(\rho ; t) \delta_{m, k}+\mathcal{E}^{(2)}(\rho ; t) \delta_{n, l}$. 

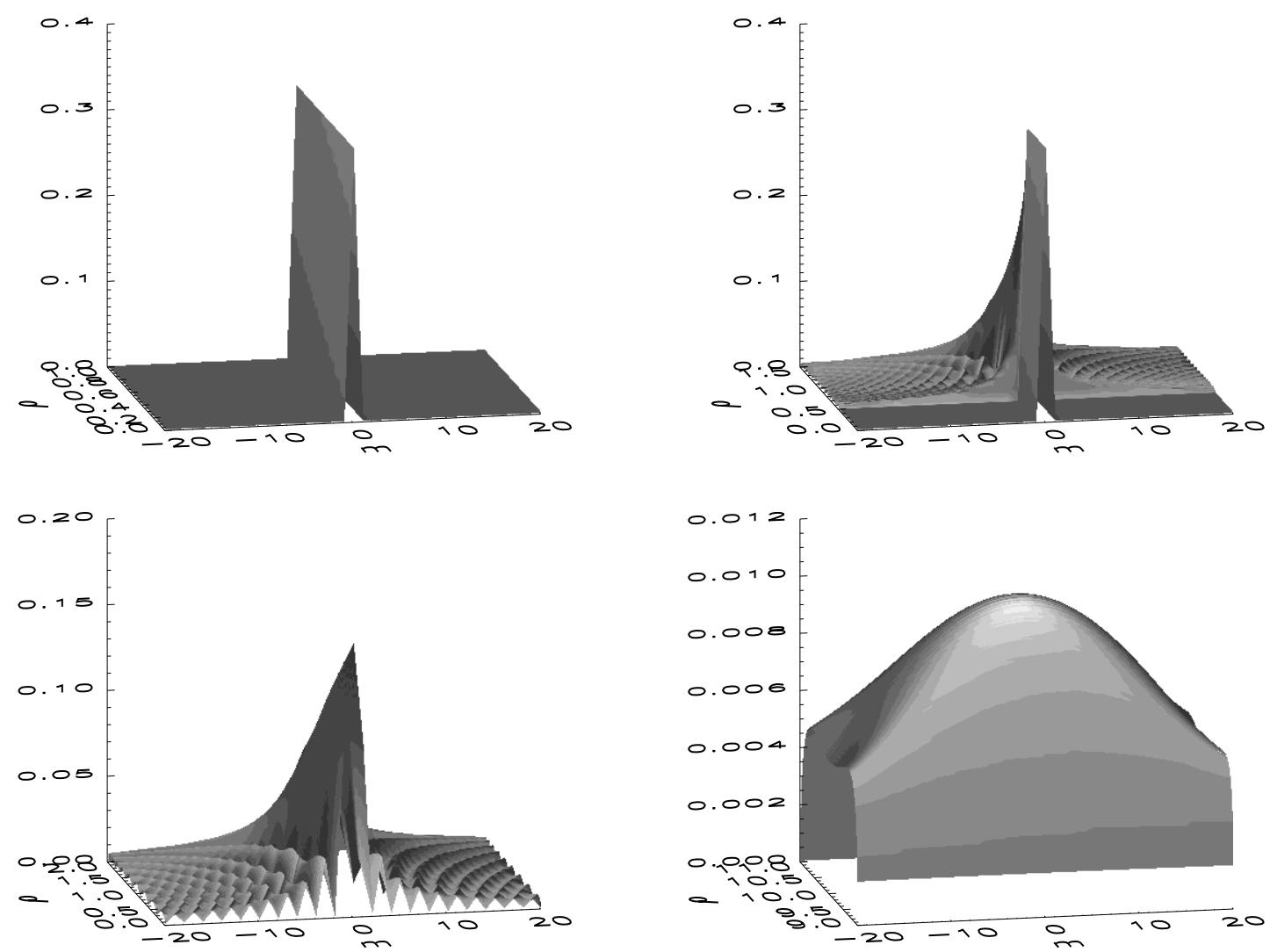

Fig. 12. A circular source of radius $\rho_{*}=1$ with an intrinsic POAM of $k=0$. The pointing offsets are $\rho_{c}=0.0,0.5,1.0$, and 10.0 (upper left, upper right, lower left, and lower right). As the pointing offset increases, the non-zero pointing POAM.

which yields the correlation spectrum

$I_{m, n}(\rho)=\mathcal{I}^{(1,1)}(\rho) \delta_{m, k} \delta_{n, k}+\mathcal{I}^{(2,2)}(\rho) \delta_{m, l} \delta_{n, l}+\mathcal{I}^{(1,2)}(\rho) \delta_{m, k} \delta_{n, l}+\mathcal{I}^{(1,2), *}(\rho) \delta_{m, l} \delta_{n, k}$

and the rancor spectrum

$\mathcal{I}_{m}(\rho)=\left[\mathcal{I}^{(1,1)}(\rho)+\mathcal{I}^{(2,2)}(\rho)\right] \delta_{m, 0}+\mathcal{I}^{(1,2)}(\rho) \delta_{m, k-l}+\mathcal{I}^{(1,2), *}(\rho) \delta_{m, l-k}$

Sources containing multiple vortices exhibit intrinsic POAM and possibly structure POAM if the states $k$ and $l$ are correlated. They are characterized by unrancored and unrancored terms, resulting from the $\phi$-independent and $\phi$-dependent behaviors.

The last POAM class can be demonstrated by shifting a single vortex state $k$ from the FOV origin by $\left(\alpha_{c}, \delta_{c}\right)$,

$E(\overrightarrow{\boldsymbol{\Omega}} ; t)=\mathcal{E}\left(\rho^{\prime}\left(\rho, \phi ; \rho_{c}, \phi_{c}\right) ; t\right) \mathrm{e}^{j k \phi^{\prime}\left(\rho, \phi ; \rho_{c}, \phi_{c}\right)}=\mathcal{E}\left(\sqrt{\rho^{2}+\rho_{c}^{2}-2 \rho \rho_{c} \cos \left(\phi-\phi_{c}\right)} ; t\right) \mathrm{e}^{j k \tan ^{-1} \frac{\rho \sin \phi-\rho_{c} \sin \phi_{c}}{\rho \cos \phi-\rho_{c} \cos \phi_{c}}}$,

where $\rho_{c}=\sqrt{\alpha_{c}^{2}+\delta_{c}^{2}}$, and $\phi_{c}=\tan ^{-1}\left(\delta_{c} / \alpha_{c}\right)$. The intensity is

$I(\overrightarrow{\mathbf{\Omega}})=I\left(\sqrt{\rho^{2}+\rho_{c}^{2}-2 \rho \rho_{c} \cos \left(\phi-\phi_{c}\right)}\right)$.

This electric field leads to the state spectrum

$E_{m}(\rho ; t)=\frac{1}{2 \pi} \int_{\phi_{-}}^{\phi_{+}} \mathrm{d} \phi \mathrm{e}^{-j m \phi} \mathcal{E}\left(\sqrt{\rho^{2}+\rho_{c}^{2}-2 \rho \rho_{c} \cos \left(\phi-\phi_{c}\right)} ; t\right) \mathrm{e}^{j k \tan ^{-1} \frac{\rho \sin \phi-\rho_{c} \sin \phi_{c}}{\rho \cos \phi-\rho_{c} \cos \phi_{c}}}$,

where the

$\phi_{ \pm}=\phi_{c} \pm \cos ^{-1} \frac{\rho^{2}+\rho_{c}^{2}-\rho_{c}^{2}}{2 \rho_{c} \rho}$

are the integration limits. This shifting procedure is not the same as the one in Sect. 2.2. Here, the source is shifted with respect to the FOV center, but we are not changing the reference point for calculating POAM. In Figs. 12 and 13, I give surface plots for the magnitude of the observed state $\left|E_{m}(\rho ; t)\right|$ as a function of radius $\rho$ for intrinsic $k=0$ and $k=5$ states. The electric field is constant with a magnitude of unity, or $|\mathcal{E}(\rho ; t)|=1$. As the offset increases, $\phi$ dependence is introduced and the state $k$ diverges to multiple states $m$. This effect is direct evidence for pointing POAM. 

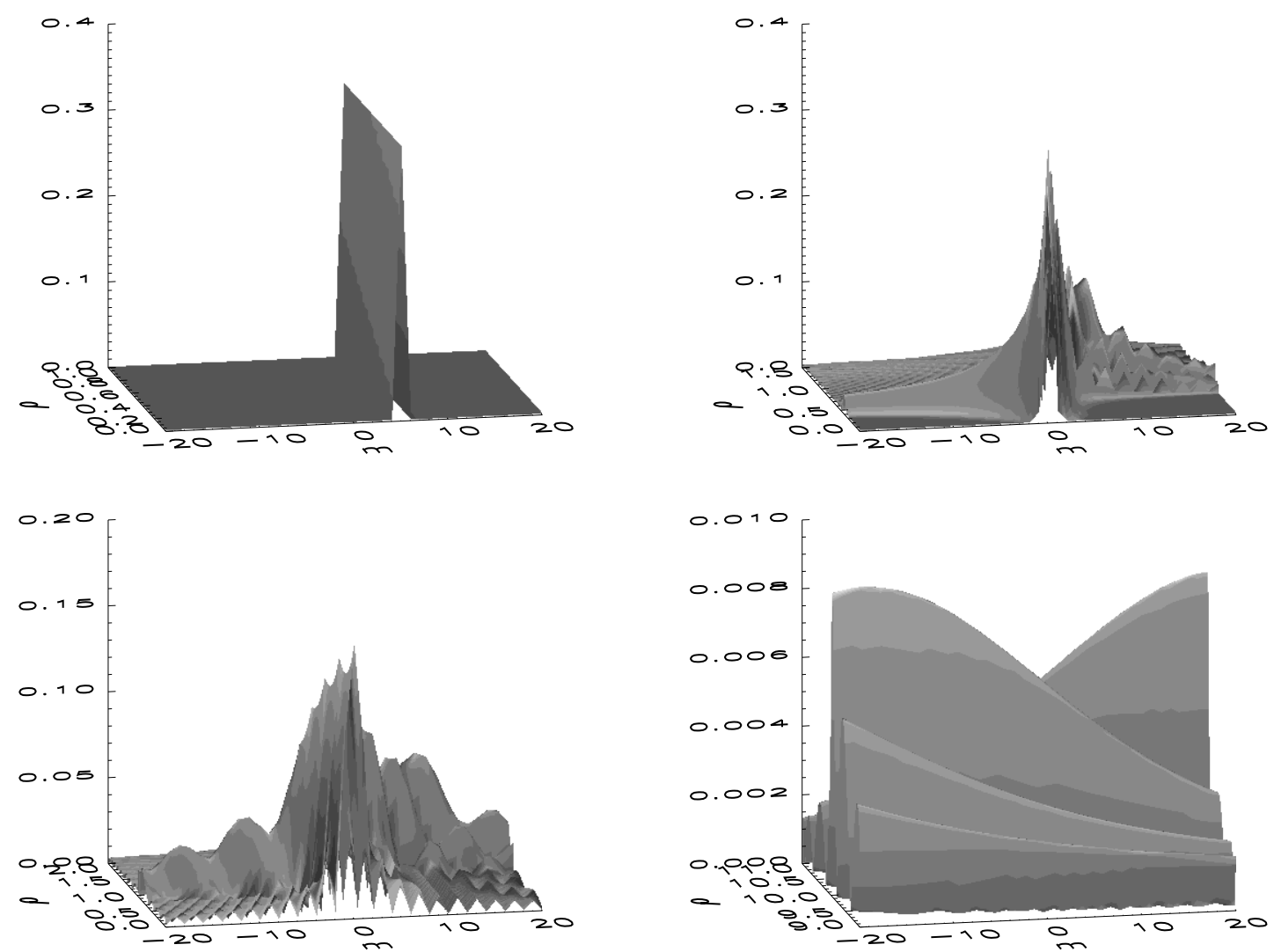

Fig. 13. A circular source of radius $\rho_{*}=1$ with an intrinsic POAM of $k=5$. The pointing offsets are $\rho_{c}=0.0,0.5,1.0$, and 10.0 (upper left, upper right, lower left, and lower right). As the pointing offset increases, the non-zero pointing POAM increases with respect to the $m=5$ state.

The correlation spectrum,

$I_{m, n}(\rho)=\left\langle\frac{1}{2} E_{m}(\rho ; t) E_{n}^{*}(\rho ; t)\right\rangle$,

cannot be simplified. The rancor spectrum is

$\mathcal{I}_{m}(\rho)=\frac{1}{2 \pi} \int_{\phi_{-}}^{\phi_{+}} \mathrm{d} \phi \mathrm{e}^{-j m \phi} \mathcal{I}\left(\sqrt{\rho^{2}+\rho_{c}^{2}-2 \rho \rho_{c} \cos \left(\phi-\phi_{c}\right)}\right)$.

In Fig. 14, I present the rancor spectra as surface plots. They are the same for both $k=0$ and $k=5$. As a matter of fact, they look the same as the $k=0$ state magnitude plots, apart from the scale factor. For a zero offset, the observed rancor $m$ is zero for all radii, which is identical to the intrinsic rancor. As the offset increases, the observed rancor spectrum deviates more and more from zero intrinsic rancor.

Structure and pointing POAM are intimately related. Changing the offset introduces a $\phi$ dependence, thus adding structure. Consider the electric field of a binary star, with each star containing no intrinsic POAM in their own coordinate systems. The observed POAM depends on where the observer decides to place the FOV origin, which could be at the center of star 1, the center of star 2, the system COL, etc. As the stars orbit each other, the rancor changes and can be measured by the limited rancorimeter (Sect. 9.2).

\section{Instrumental POAM}

Instrumental POAM is produced by all types aberrations. It is analogous to instrumental PSAM, which is more commonly known as instrumental polarization. For meaningful observations, instrumental POAM must be calibrated. It is caused by either optical aberrations or atmospheric turbulence. The former can be compensated by adaptive optics or modeled/removed in data reduction. The latter may require EAO (Boccaletti et al. 2002; Crepp et al. 2007), depending on the application.

\subsection{Classical forms and POAM expansions}

Consider a single telescope (Sect. 6). The amplitude/phase aberration function of imperfect optics can be expressed as the sum of a real constant plus a complex perturbation, or

$\mathcal{D}(\overrightarrow{\mathcal{R}}, \overrightarrow{\mathbf{\Omega}})=\mathcal{D}_{0}+\Delta \mathcal{D}(\overrightarrow{\mathcal{R}}, \overrightarrow{\mathbf{\Omega}})$ 

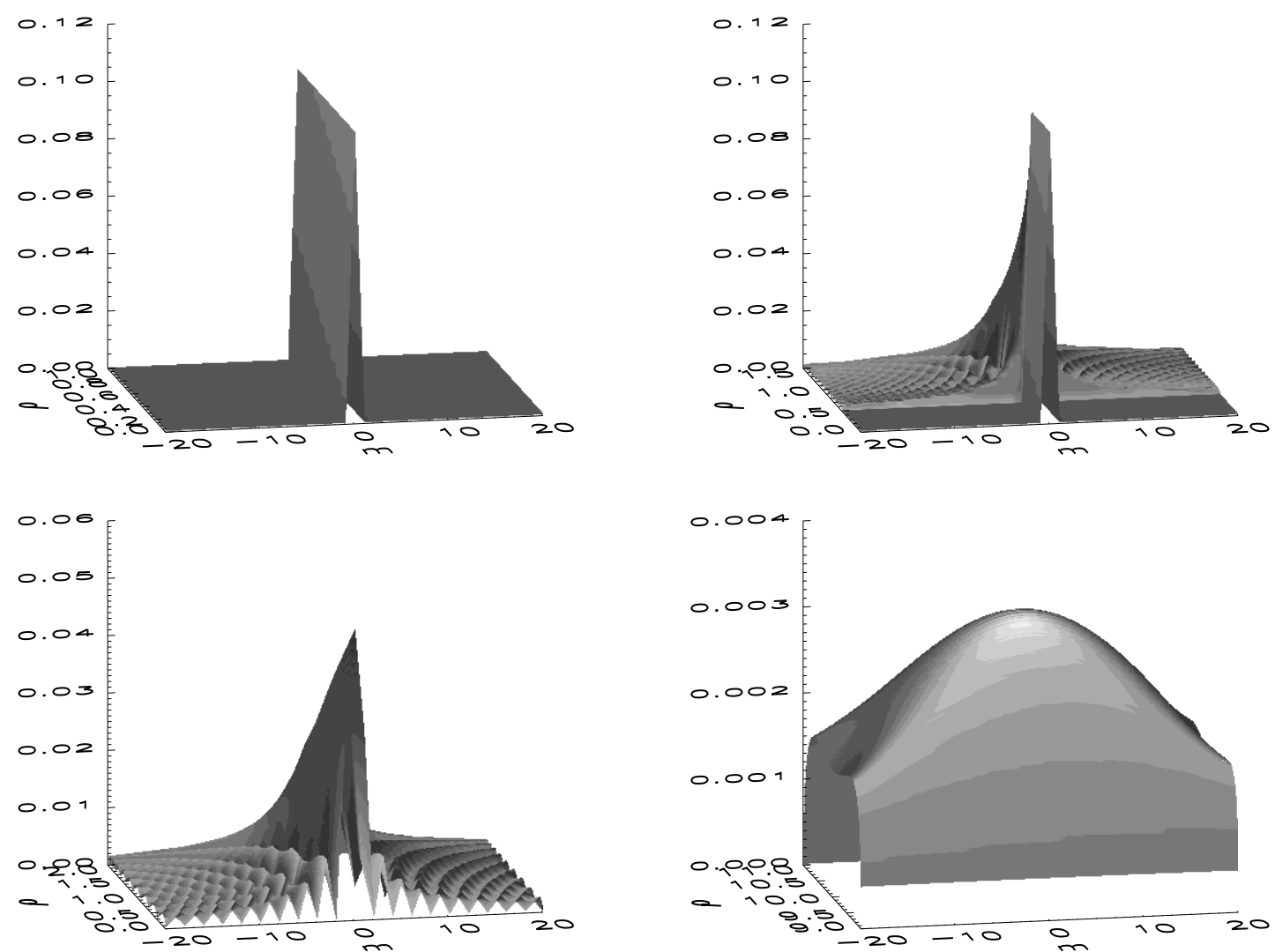

Fig. 14. A circular source of radius $\rho_{*}=1$ with an intrinsic rancor of zero. The pointing offsets are $\rho_{c}=0.0,0.5,1.0$, and 10.0 (upper left, upper right, lower left, and lower right). As the pointing offset increases, the non-zero pointing rancor increases.

I choose $\Delta \mathcal{D}(\overrightarrow{\mathcal{R}}, \overrightarrow{\boldsymbol{\Omega}})=0$ for $\overrightarrow{\mathcal{R}}=\overrightarrow{\boldsymbol{\Omega}}=0$. Since the DF (Eq. (23b)) acts linearly upon the amplitude/phase aberration function, the result consists of an unperturbed DF part (Eq. (25a)) plus a perturbed DF part

$D(\overrightarrow{\mathbf{r}}, \overrightarrow{\boldsymbol{\Omega}})=\mathbf{D}(\overrightarrow{\mathbf{r}}, \overrightarrow{\boldsymbol{\Omega}})+\Delta D(\overrightarrow{\mathbf{r}}, \overrightarrow{\boldsymbol{\Omega}})=\mathcal{D}_{0} \mathcal{J}_{1}\left[2 \pi \kappa R_{\mathrm{tel}} \Gamma(\overrightarrow{\mathbf{r}}, \overrightarrow{\boldsymbol{\Omega}})\right]+\frac{1}{A_{\mathrm{ep}}} \int \mathrm{d}^{2} \mathcal{R} \mathrm{e}^{-j 2 \pi \kappa M\left[\frac{\vec{r}}{\mathrm{\theta}}-\overrightarrow{\mathbf{s}}_{x y}(\overrightarrow{\boldsymbol{\Omega}})\right] \cdot \overrightarrow{\mathcal{R}}} \Delta \mathcal{D}(\overrightarrow{\mathcal{R}}, \overrightarrow{\boldsymbol{\Omega}})$.

The overlap integral (Eq. (24a)) acts linearly upon the DF, so the result consists of an unperturbed electric-field part plus a perturbed electric-field part

$E(\overrightarrow{\mathbf{r}} ; t)=\mathbf{E}(\overrightarrow{\mathbf{r}} ; t)+\Delta E(\overrightarrow{\mathbf{r}} ; t)=\int \mathrm{d}^{2} \Omega[\mathbf{D}(\overrightarrow{\mathbf{r}}, \overrightarrow{\boldsymbol{\Omega}})+\Delta D(\overrightarrow{\mathbf{r}}, \overrightarrow{\boldsymbol{\Omega}})] E(\overrightarrow{\boldsymbol{\Omega}} ; t)$.

When this equation is substituted into Table 1, it is clear that the expansions also act linearly, which means that each input sensitivity, output sensitivity, and input/output gain consists of an unperturbed part (Sect. 6.2) plus a perturbed part.

When I calculate the squared magnitude of an aberrated telescope DF, I obtain the telescope PSF

$P(\overrightarrow{\mathbf{r}}, \overrightarrow{\boldsymbol{\Omega}})=\mathbf{P}(\overrightarrow{\mathbf{r}}, \overrightarrow{\boldsymbol{\Omega}})+\Delta P(\overrightarrow{\mathbf{r}}, \overrightarrow{\boldsymbol{\Omega}})$,

where

$\Delta P(\overrightarrow{\mathbf{r}}, \overrightarrow{\boldsymbol{\Omega}})=2 \operatorname{Re}\left\{\mathbf{D}(\overrightarrow{\mathbf{r}}, \overrightarrow{\boldsymbol{\Omega}}) \Delta D^{*}(\overrightarrow{\mathbf{r}}, \overrightarrow{\boldsymbol{\Omega}})\right\}+|\Delta D(\overrightarrow{\mathbf{r}}, \overrightarrow{\boldsymbol{\Omega}})|^{2} \approx 2 \operatorname{Re}\left\{\mathbf{D}(\overrightarrow{\mathbf{r}}, \overrightarrow{\boldsymbol{\Omega}}) \Delta D^{*}(\overrightarrow{\mathbf{r}}, \overrightarrow{\boldsymbol{\Omega}})\right\}$.

The approximation holds when aberrations are small. Since the overlap integral (Eq. (24b)) acts linearly upon the PSF, the result consists of an unperturbed intensity part plus a perturbed intensity part

$I(\overrightarrow{\mathbf{r}})=\mathbf{I}(\overrightarrow{\mathbf{r}})+\Delta I(\overrightarrow{\mathbf{r}})=\int \mathrm{d}^{2} \Omega[\mathbf{P}(\overrightarrow{\mathbf{r}}, \overrightarrow{\mathbf{\Omega}})+\Delta P(\overrightarrow{\mathbf{r}}, \overrightarrow{\boldsymbol{\Omega}})] I(\overrightarrow{\boldsymbol{\Omega}})$.

When this equation is substituted into Table 3 , it is clear that the expansions also act linearly, which means that each input sensitivity, output sensitivity, and input/output gain consists of an unperturbed part (Sect. 6.2) plus a perturbed part. Perturbed intensities lead to perturbed integrated probabilities and POAM expectation values in the image plane, which means that external torque has been applied to the wavefronts.

In Fig. 15, I combine the three types of POAM discussed in Sect. 10 (intrinsic, structure, and pointing) along with instrumental POAM. Intrinsic and structure POAM are types of source POAM. Instrumental POAM may be divided into pointing and high-order aberrations. I have linked structure and pointing POAM, because off-axis structure and pointing both lead to tilted wavefronts. 


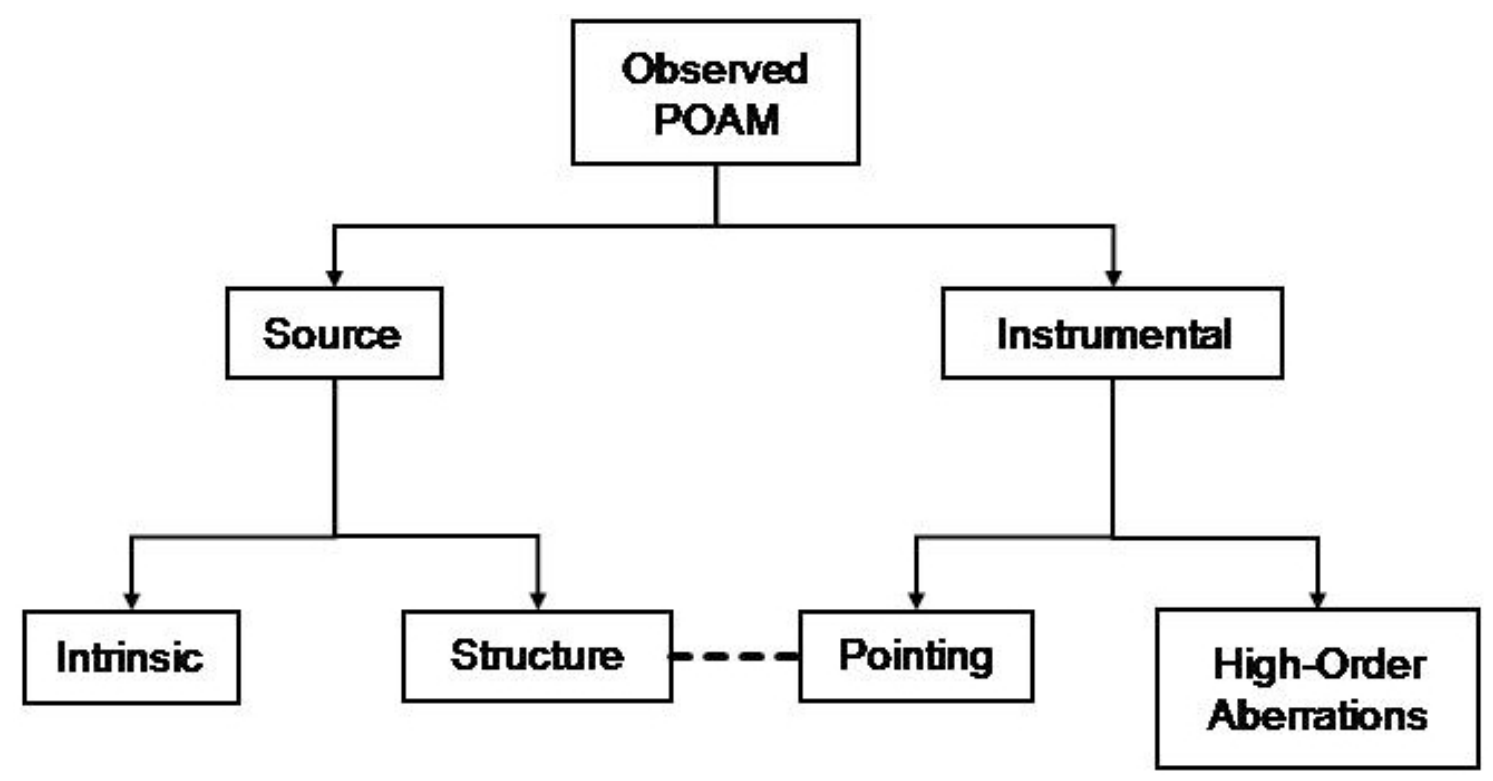

Fig. 15. The types of source and instrumental POAM. The dashed line indicates that structure and pointing POAM are related.

\subsection{Zernike polynomials}

I discuss the POAM behavior of coronagraphs in Sect. 7 and optical aberrations in Sect. 11.1. Ferrari (2007) discusses the imperfect behavior of Lyot coronagraphs in terms of Zernike polynomials. Together these statements suggest that POAM expansions of DFs can be expressed in terms Zernike polynomials (and vice versa). Zernike polynomials are frequently used by optical scientists and engineers to describe aberrations in optical systems. In fact, they can even be used to characterize an instantaneous or time-averaged rms wavefront corrugated by atmospheric turbulence. In this section, I demonstrate that Zernike polynomials are closely related to the perturbed DFs described in Sect. 11.1.

If the amplitude/phase aberration function contains only phase errors, then

$\mathcal{D}(\overrightarrow{\mathcal{R}}, \overrightarrow{\boldsymbol{\Omega}})=\mathcal{D}_{0} \mathrm{e}^{j \Delta \Phi\left(\overrightarrow{\mathcal{R}} / R_{\mathrm{ep}}, \overrightarrow{\boldsymbol{\Omega}}\right)} \approx \mathcal{D}_{0}+j \mathcal{D}_{0} \Delta \Phi\left(\overrightarrow{\mathcal{R}} / R_{\mathrm{ep}}, \overrightarrow{\boldsymbol{\Omega}}\right)$

where $\Delta \Phi\left(\overrightarrow{\mathcal{R}} / R_{\mathrm{ep}}, \overrightarrow{\boldsymbol{\Omega}}\right)$ is the phase aberration function. I assume that the phase aberrations are small. The second term represents the perturbation of Eq. (72a). When it is substituted into Eq. (72b), I obtain the DF perturbation

$$
\begin{aligned}
\Delta D(\overrightarrow{\mathbf{r}}, \overrightarrow{\boldsymbol{\Omega}}) & =j \mathcal{D}_{0} \frac{1}{A_{\mathrm{ep}}} \int \mathrm{d}^{2} \mathcal{R} \mathrm{e}^{-j 2 \pi \kappa M\left[\frac{\overrightarrow{\mathrm{r}}}{\Theta}-\overrightarrow{\mathbf{s}}_{x y}(\overrightarrow{\boldsymbol{\Omega}})\right] \cdot \overrightarrow{\mathcal{R}}} \Delta \Phi\left(\overrightarrow{\mathcal{R}} / R_{\mathrm{ep}}, \overrightarrow{\boldsymbol{\Omega}}\right) \\
& =j \mathcal{D}_{0} 2 \int_{0}^{1} \mathrm{~d} u u \frac{1}{2 \pi} \int_{0}^{2 \pi} \mathrm{d} \psi \mathrm{e}^{-j 2 \pi \kappa R_{\mathrm{tel}} \Gamma(\overrightarrow{\mathbf{r}}, \overrightarrow{\boldsymbol{\Omega}}) u \cos [\psi-\gamma(\overrightarrow{\mathbf{r}}, \overrightarrow{\boldsymbol{\Omega}})]} \Delta \Phi(\overrightarrow{\mathbf{u}}, \overrightarrow{\boldsymbol{\Omega}}),
\end{aligned}
$$

where $\overrightarrow{\mathbf{u}}=\overrightarrow{\mathcal{R}} / R_{\mathrm{ep}}$, and

$\gamma(\overrightarrow{\mathbf{r}}, \overrightarrow{\boldsymbol{\Omega}})=\tan ^{-1} \frac{\frac{r}{\Theta} \sin \xi-\rho \sin \phi}{\frac{r}{\Theta} \cos \xi-\rho \cos \phi}$

is the angle corresponding to $\Gamma(\overrightarrow{\mathbf{r}}, \overrightarrow{\mathbf{\Omega}})($ Sect. 6).

To study the aberrations, I express $\Delta \Phi(\overrightarrow{\mathbf{u}}, \overrightarrow{\boldsymbol{\Omega}})$ in terms of a Zernike expansion,

$\Delta \Phi(\overrightarrow{\mathbf{u}}, \overrightarrow{\boldsymbol{\Omega}})=\sum_{m=0}^{\infty} \sum_{\substack{n=m, n-m=\text { even }}}^{\infty} R_{m, n}(u)\left[a_{m, n}(\overrightarrow{\boldsymbol{\Omega}}) \cos m \psi+b_{m, n}(\overrightarrow{\boldsymbol{\Omega}}) \sin m \psi\right]=\sum_{m=0}^{\infty} \sum_{\substack{n=m, n-m=\text { even }}}^{\infty} R_{m, n}(u)\left[F_{m, n}(\overrightarrow{\boldsymbol{\Omega}}) \mathrm{e}^{j m \psi}+F_{m, n}^{*}(\overrightarrow{\boldsymbol{\Omega}}) \mathrm{e}^{-j m \psi}\right]$

where the $a_{m, n}(\overrightarrow{\mathbf{\Omega}})$ and $b_{m, n}(\overrightarrow{\mathbf{\Omega}})$ are real coefficients, the $F_{m, n}(\overrightarrow{\mathbf{\Omega}})=\frac{1}{2}\left[a_{m, n}(\overrightarrow{\mathbf{\Omega}})-j b_{m, n}(\overrightarrow{\mathbf{\Omega}})\right]$ are complex coefficients, and the $R_{m, n}(u)$ are Zernike radial functions (Wyant \& Creath 1992). Valid $u$ arguments range between 0 and 1 , and $R_{m, n}(1)=1$. Note that $a_{0,0}(\overrightarrow{\mathbf{\Omega}})$ $=b_{0,0}(\overrightarrow{\mathbf{\Omega}})=F_{0,0}(\overrightarrow{\mathbf{\Omega}})=0$. When I substitute this equation into Eq. (75a), I obtain

$\Delta D(\overrightarrow{\mathbf{r}}, \overrightarrow{\boldsymbol{\Omega}})=\mathcal{D}_{0} \sum_{m=0}^{\infty} \sum_{\substack{n=m, n-m=\text { even }}}^{\infty}(-1)^{m} j^{n+1} \mathcal{J}_{n+1}\left[2 \pi \kappa R_{\mathrm{tel}} \Gamma(\overrightarrow{\mathbf{r}}, \overrightarrow{\boldsymbol{\Omega}})\right]\left[F_{m, n}(\overrightarrow{\boldsymbol{\Omega}}) \mathrm{e}^{j m \gamma(\overrightarrow{\mathbf{r}}, \overrightarrow{\boldsymbol{\Omega}})}+F_{m, n}^{*}(\overrightarrow{\boldsymbol{\Omega}}) \mathrm{e}^{-j m \gamma(\overrightarrow{\mathbf{r}}, \overrightarrow{\mathbf{\Omega}})}\right]$, 
where $\mathcal{J}_{k}(x)=2 J_{k}(x) / x$. I derive this equation in Appendix $\mathrm{J}$.

For the sake of simplicity, I consider only a small circular source at the FOV, which means that $\Gamma(\overrightarrow{\mathbf{r}}, \overrightarrow{\boldsymbol{\Omega}}) \approx \Gamma(\overrightarrow{\mathbf{r}}, \overrightarrow{\mathbf{0}})=r / \Theta$ and $\gamma(\overrightarrow{\mathbf{r}}, \overrightarrow{\boldsymbol{\Omega}}) \approx \gamma(\overrightarrow{\mathbf{r}}, \overrightarrow{\mathbf{0}})=\xi$. With this assumption, calculating the input sensitivities or input/output gains of the DF are not advantageous, so I expand it in terms of the output sensitivities

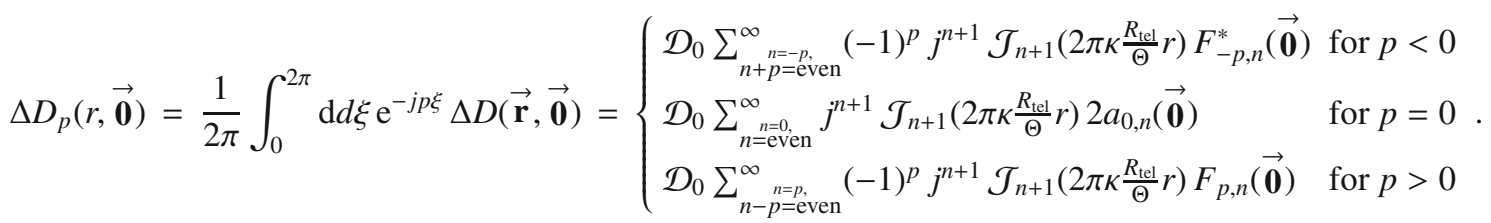

Each component manifests itself as concentric rings. If there are no aberrations (all $F_{k, l}(\overrightarrow{\mathbf{0}})$ are 0$)$, then the total perturbation is identically zero, as expected.

\section{Conclusion}

In this paper, I adopt a semi-classical/semi-quantum model to describe astronomical POAM. Electric fields are expanded into azimuthal Fourier components, which represent pure vortex states. Similarly, intensities can be expanded in terms of correlations or rancors. Each rancor is an azimuthal Fourier component of the intensity, as well as the sum of correlations with the same rancor. There are loose analogies between POAM and PSAM.

I propagated the electric-field and intensity POAM expansions from celestial sphere through free space and instrument to a specific plane. The expressions, which include POAM expansions of the diffraction and point-spread functions, are very generic and adaptable to a wide variety of instruments. I employ these examples to demonstrate their utility (in order of difficulty): free space, single telescopes, coronagraphs, interferometers, and rancorimeters. The electric-field expansions can be used with both spatially coherent and incoherent sources, while the intensity expansions should only be used with spatially incoherent sources.

Propagation through free space and an unaberrated telescope applies no external torque to the wavefronts, so the integrated probabilities and POAM expectation value are conserved. Conversely, propagation through an aberrated telescope applies external torque, which may be expressed in terms of Zernike polynomials. Coronagraphs modulate the zero-order state from the central star to produce a null. An interferometer can filter POAM without additional optics. For example, single-Bracewell interferometers track $180^{\circ}$ from the central fringe, eliminating the even states and producing a null. A limited rancorimeter, which measures only rancors, can be created by placing a rotating focal-plane wedge mask into a coronagraph. As a possible application, I use this instrument to perform super-Rayleigh modeling of an unresolved binary star.

POAM on the celestial sphere can be classified as intrinsic, structure, or pointing. Pointing and structure POAM are related. Sources can contain intrinsic and structure POAM simultaneously. Instrumental POAM, which must be calibrated, includes optical aberrations (pointing and high-order) and atmospheric turbulence.

POAM is a relatively new field within astronomy. The mathematics in this paper should be used as a starting point for future POAM research. Topics include: 1) making a priori predictions for the intrinsic POAM of astronomical sources; 2) designing ground- and space-based POAM-measuring instruments; 3) understanding existing instruments in terms of POAM; 4) minimizing the effects of random noise on POAM; and 5) calibrating instrumental (optical aberrations and atmospheric turbulence) POAM.

Acknowledgements. The author gratefully acknowledges Martin Harwit for his support and encouragement, as well as the referees Immo Appenzeller and Steven N. Shore for their critical review of this manuscript.

\section{Appendix A: Calculating the azimuthal derivative of the aberration function}

Equation (1c), which is the definition of $\Phi(\overrightarrow{\mathbf{H}} ; t)$, contains functions of $\epsilon$, so chain-rule differentiation is required,

$$
\frac{\partial \Phi(\overrightarrow{\mathbf{H}})}{\partial \epsilon}=\left\langle\frac{\partial \Phi(\overrightarrow{\mathbf{H}} ; t)}{\partial \epsilon}\right\rangle=\left\langle\frac{\partial}{\partial \epsilon}\left[\tan ^{-1} \frac{\operatorname{Im} E(\overrightarrow{\mathbf{H}} ; t)}{\operatorname{Re} E(\overrightarrow{\mathbf{H}} ; t)}\right]\right\rangle=\left\langle\frac{1}{1+\left[\frac{\operatorname{Im} E(\overrightarrow{\mathbf{H}} ; t)}{\operatorname{Re} E(\overrightarrow{\mathbf{H}} ; t)}\right]^{2}} \frac{\partial\left[\frac{\operatorname{Im} E(\overrightarrow{\mathbf{H}} ; t)}{\operatorname{Re} E(\overrightarrow{\mathbf{H}} ; t)}\right]}{\partial \epsilon}\right\rangle=\frac{1}{I(\overrightarrow{\mathbf{H}})}\left\langle\frac{1}{2}\left[\operatorname{Re}^{2} E(\overrightarrow{\mathbf{H}} ; t)\right] \frac{\partial\left[\frac{\operatorname{Im} E(\overrightarrow{\mathbf{H}} ; t)}{\operatorname{Re} E(\overrightarrow{\mathbf{H}} ; t)}\right]}{\partial \epsilon}\right\rangle,
$$

where I assume that $|E(\overrightarrow{\mathbf{H}} ; t)|^{2}=\operatorname{Re}^{2}[E(\overrightarrow{\mathbf{H}} ; t)]+\operatorname{Im}^{2}[E(\overrightarrow{\mathbf{H}} ; t)] \approx$ constant compared to the other factors. The other derivative, using the quotient rule, is

$$
\begin{aligned}
\frac{\partial\left[\frac{\operatorname{Im} E(\overrightarrow{\mathbf{H}} ; t)}{\operatorname{Re} E(\overrightarrow{\mathbf{H}} ; t)}\right]}{\partial \epsilon} & =\frac{1}{\operatorname{Re}^{2} E(\overrightarrow{\mathbf{H}} ; t)}\left\{[\operatorname{Re} E(\overrightarrow{\mathbf{H}} ; t)]\left[\frac{\operatorname{Im} \partial E(\overrightarrow{\mathbf{H}} ; t)}{\partial \epsilon}\right]-[\operatorname{Im} E(\overrightarrow{\mathbf{H}} ; t)]\left[\frac{\operatorname{Re} \partial E(\overrightarrow{\mathbf{H}} ; t)}{\partial \epsilon}\right]\right\} \\
& =\frac{-1}{\operatorname{Re}^{2} E(\overrightarrow{\mathbf{H}} ; t)} \operatorname{Im}\left[E(\overrightarrow{\mathbf{H}} ; t) \frac{\partial E^{*}(\overrightarrow{\mathbf{H}} ; t)}{\partial \epsilon}\right] .
\end{aligned}
$$


Substituting Eq. (A.2) into Eq. (A.1), I obtain

$\frac{\partial \Phi(\overrightarrow{\mathbf{H}})}{\partial \epsilon}=-\frac{1}{I(\overrightarrow{\mathbf{H}})} \operatorname{Im}\left\langle\frac{1}{2} E(\overrightarrow{\mathbf{H}} ; t) \frac{\partial E^{*}(\overrightarrow{\mathbf{H}} ; t)}{\partial \epsilon}\right\rangle$.

When I plug Eq. (9a) into Eq. (A.3), I find that

$$
\begin{aligned}
\frac{\partial \Phi(\overrightarrow{\mathbf{H}})}{\partial \epsilon} & =-\frac{1}{I(\overrightarrow{\mathbf{H}})} \operatorname{Im}\left\langle\frac{1}{2} \sum_{m=-\infty}^{\infty} E_{m}(H ; t) \mathrm{e}^{j m \epsilon} \frac{\partial}{\partial \epsilon}\left[\sum_{n=-\infty}^{\infty} E_{n}^{*}(H ; t) \mathrm{e}^{-j n \epsilon}\right]\right\rangle=\frac{1}{I(\overrightarrow{\mathbf{H}})} \operatorname{Im}\left[-j \sum_{m=-\infty}^{\infty} \sum_{n=-\infty}^{\infty} n\left\langle\frac{1}{2} E_{m}(H ; t) E_{n}^{*}(H ; t)\right\rangle \mathrm{e}^{j(m-n) \epsilon}\right] \\
& =\frac{1}{I(\overrightarrow{\mathbf{H}})} \operatorname{Re} \sum_{m=-\infty}^{\infty} \sum_{n=-\infty}^{\infty} n I_{m, n}(H) \mathrm{e}^{j(m-n) \epsilon}
\end{aligned}
$$

Note that the POAM expansion of the electric field makes the $\epsilon$ derivatives trivial.

If the link between the specific plane and the celestial sphere is known, via the DF, the azimuthal derivative of the aberration function can be written as

$\frac{\partial \Phi(\overrightarrow{\mathbf{N}} ; \overrightarrow{\mathbf{a}})}{\partial v}=-\frac{1}{I(\overrightarrow{\mathbf{N}})} \operatorname{Im} \int \mathrm{d}^{2} \Omega D(\overrightarrow{\mathbf{N}}, \overrightarrow{\boldsymbol{\Omega}} ; \overrightarrow{\mathbf{a}}) \frac{\partial D^{*}(\overrightarrow{\mathbf{N}}, \overrightarrow{\boldsymbol{\Omega}} ; \overrightarrow{\mathbf{a}})}{\partial v} I(\overrightarrow{\boldsymbol{\Omega}})=\frac{1}{I(\overrightarrow{\mathbf{N}} ; \overrightarrow{\mathbf{a}})} \operatorname{Re} \sum_{p=-\infty}^{\infty} \sum_{q=-\infty}^{\infty} q I_{p, q}(N ; \overrightarrow{\mathbf{a}}) \mathrm{e}^{j(p-q) v}$

for $\overrightarrow{\mathbf{H}} \rightarrow \overrightarrow{\mathbf{N}}$. This final expression is valid only for spatially incoherent sources. The DF and intensity on the celestial sphere are expanded according to the formulae in Sects. 3 and 4 to obtain the second part of the equation, which has the same form as Eq. (A.4).

\section{Appendix B: Calculating the rancor components in terms of the correlation components}

Substituting Eq. (10a) into Eq. (12b), I obtain

$\mathcal{I}_{k}(H)=\frac{1}{2 \pi} \int_{0}^{2 \pi} \mathrm{d} \epsilon \mathrm{e}^{-j k \epsilon} \sum_{m=-\infty}^{\infty} \sum_{n=-\infty}^{\infty} I_{m, n}(H) \mathrm{e}^{j(m-n) \epsilon}=\sum_{m=-\infty}^{\infty} \sum_{n=-\infty}^{\infty} I_{m, n}(H)\left[\frac{1}{2 \pi} \int_{0}^{2 \pi} \mathrm{d} \epsilon \mathrm{e}^{-j(k-m+n) \epsilon}\right]$.

The integral in the square brackets is simply the Kronecker delta $\delta_{n, m-k}$, which means that one of the sums in Eq. (B.1) disappears, or

$I_{k}(H)=\sum_{m=-\infty}^{\infty} I_{m, m-k}(H)$

The index $k$ is called the rancor index. It is possible to reverse this derivation by substituting Eqs. (9a) and (10b) back into Eq. (12b),

$$
\begin{aligned}
\mathcal{I}_{k}(H) & =\sum_{m=-\infty}^{\infty}\left\langle\frac{1}{2} E_{m}(H ; t) E_{m-k}^{*}(H ; t)\right\rangle=\sum_{m=-\infty}^{\infty}\left\langle\frac{1}{2} \frac{1}{2 \pi} \int_{0}^{2 \pi} \mathrm{d} \epsilon \mathrm{e}^{-j m \epsilon} E(\overrightarrow{\mathbf{H}} ; t) \frac{1}{2 \pi} \int_{0}^{2 \pi} \mathrm{d} \epsilon^{\prime} \mathrm{e}^{j(m-k) \epsilon^{\prime}} E^{*}\left(\overrightarrow{\mathbf{H}^{\prime}} ; t\right)\right\rangle \\
& =\frac{1}{2 \pi} \int_{0}^{2 \pi} \mathrm{d} \epsilon \frac{1}{2 \pi} \int_{0}^{2 \pi} \mathrm{d} \epsilon^{\prime} \mathrm{e}^{-j k \epsilon^{\prime}}\left[\sum_{m=-\infty}^{\infty} \mathrm{e}^{-j m\left(\epsilon-\epsilon^{\prime}\right)}\right]\left\langle\frac{1}{2} E(\overrightarrow{\mathbf{H}} ; t) E^{*}\left(\overrightarrow{\mathbf{H}^{\prime}} ; t\right)\right\rangle
\end{aligned}
$$

where $\overrightarrow{\mathbf{H}}^{\prime}=\left(H \cos \epsilon^{\prime}, H \sin \epsilon^{\prime}\right)$. The quantity in the square brackets is $2 \pi \delta\left(\epsilon-\epsilon^{\prime}\right)$ and the quantity in the angular brackets is $I(\overrightarrow{\mathbf{H}})$ (after the Dirac delta function is applied within the integral).

\section{Appendix C: Calculating POAM quantities in the specific plane}

In this appendix, I make extensive use of the equations and tables of Sects. 3 and 4. One must insure not to confuse the substitutions of vectors $\overrightarrow{\mathbf{N}}$ (the vector in the specific plane) and $\overrightarrow{\boldsymbol{\Omega}}$ (the vector on the celestial sphere) as $\overrightarrow{\mathbf{H}}$ (the vector in the specific plane), depending on the context. All of the electric-field POAM expansions can be used for spatially incoherent and coherent electric fields on the celestial sphere. All of the intensity POAM expansions, however, can be used only with spatially incoherent sources on the celestial sphere. There are multiple ways to derive these equations. I chose the simplest.

The first electric-field POAM expansion (Table 1) is obtained when Eq. (9a) is substituted into Eq. (24a),

$E(\overrightarrow{\mathbf{N}} ; \overrightarrow{\mathbf{a}}, t)=\int \mathrm{d}^{2} \Omega D(\overrightarrow{\mathbf{N}}, \overrightarrow{\boldsymbol{\Omega}} ; \overrightarrow{\mathbf{a}}) \sum_{m=-\infty}^{\infty} E_{m}(\rho ; t) \mathrm{e}^{j m \phi}=2 \pi \int_{0}^{\infty} \mathrm{d} \rho \rho\left[\frac{1}{2 \pi} \int_{0}^{2 \pi} \mathrm{d} \phi \mathrm{e}^{j m \phi} D(\overrightarrow{\mathbf{N}}, \overrightarrow{\boldsymbol{\Omega}} ; \overrightarrow{\mathbf{a}})\right] E_{m}(\rho ; t)$ 
The quantity in the square brackets is the input sensitivity $D^{-m}(\overrightarrow{\mathbf{N}}, \rho ; \overrightarrow{\mathbf{a}})$ defined in Table 2 . The second electric-field POAM expansion is obtained by substituting Eq. (24a) back into Eq. (9a),

$$
\begin{aligned}
E(\overrightarrow{\mathbf{N}} ; \overrightarrow{\mathbf{a}}, t) & =\sum_{p=-\infty}^{\infty} \frac{1}{2 \pi} \int_{0}^{2 \pi} \mathrm{d} v^{\prime} \mathrm{e}^{-j p v^{\prime}} \int \mathrm{d}^{2} \Omega D\left(\overrightarrow{\mathbf{N}^{\prime}}, \overrightarrow{\boldsymbol{\Omega}} ; \overrightarrow{\mathbf{a}}\right) E(\overrightarrow{\boldsymbol{\Omega}} ; t) \mathrm{e}^{j p v} \\
& =\sum_{p=-\infty}^{\infty}\left\{\int \mathrm{d}^{2} \Omega\left[\frac{1}{2 \pi} \int_{0}^{2 \pi} \mathrm{d} v^{\prime} \mathrm{e}^{-j p v^{\prime}} D\left(\overrightarrow{\mathbf{N}^{\prime}}, \overrightarrow{\boldsymbol{\Omega}} ; \overrightarrow{\mathbf{a}}\right)\right] E(\overrightarrow{\boldsymbol{\Omega}} ; t)\right\} \mathrm{e}^{j p v},
\end{aligned}
$$

where $\overrightarrow{\mathbf{N}^{\prime}}=\left(N \cos v^{\prime}, N \sin v^{\prime}\right)$. The quantity within the curly brackets is the specific-plane POAM $E_{p}(N ; \overrightarrow{\mathbf{a}}, t)$, and the quantity within the square brackets is the output sensitivity $D_{p}(N ; \overrightarrow{\mathbf{\Omega}} ; \overrightarrow{\mathbf{a}})$ defined in Table 2. The third electric-field POAM expansion is obtained by substituting Eq. (9a) into the previous equation,

$$
\begin{aligned}
E(\overrightarrow{\mathbf{N}} ; \overrightarrow{\mathbf{a}}, t) & =\sum_{p=-\infty}^{\infty}\left\{\int \mathrm{d}^{2} \Omega\left[\frac{1}{2 \pi} \int_{0}^{2 \pi} \mathrm{d} v^{\prime} \mathrm{e}^{-j p v^{\prime}} D\left(\overrightarrow{\mathbf{N}^{\prime}}, \overrightarrow{\boldsymbol{\Omega}} ; \overrightarrow{\mathbf{a}}\right)\right] \sum_{m=-\infty}^{\infty} E_{m}(\rho ; t) \mathrm{e}^{j m \phi}\right\} \mathrm{e}^{j p v} \\
& =\sum_{p=-\infty}^{\infty}\left\{\sum_{m=-\infty}^{\infty} 2 \pi \int_{0}^{\infty} \mathrm{d} \rho \rho\left[\frac{1}{2 \pi} \int_{0}^{2 \pi} \mathrm{d} \phi \mathrm{e}^{j m \phi} \frac{1}{2 \pi} \int_{0}^{2 \pi} \mathrm{d} v^{\prime} \mathrm{e}^{-j p v^{\prime}} D\left(\overrightarrow{\mathbf{N}^{\prime}}, \overrightarrow{\boldsymbol{\Omega}} ; \overrightarrow{\mathbf{a}}\right)\right] E_{m}(\rho ; t)\right\} \mathrm{e}^{j p v} .
\end{aligned}
$$

Again, the quantity within the curly brackets is the propagated-plane state $E_{p}(N ; \overrightarrow{\mathbf{a}}, t)$, and the quantity within the square brackets is the source/focal gain $D_{p}^{-m}(N, \rho ; \overrightarrow{\mathbf{a}})$ defined in Table 2 .

The first intensity POAM expansion (Table 3) is obtained when Eq. (10a) is substituted into Eq. (24b),

$I(\overrightarrow{\mathbf{N}} ; \overrightarrow{\mathbf{a}})=\int \mathrm{d}^{2} \Omega P(\overrightarrow{\mathbf{N}}, \overrightarrow{\boldsymbol{\Omega}} ; \overrightarrow{\mathbf{a}}) \sum_{m=-\infty}^{\infty} \sum_{n=-\infty}^{\infty} I_{m, n}(\rho) \mathrm{e}^{j(m-n) \phi}=\sum_{m=-\infty}^{\infty} \sum_{n=-\infty}^{\infty} 2 \pi \int_{0}^{\infty} \mathrm{d} \rho \rho\left[\frac{1}{2 \pi} \int_{0}^{2 \pi} \mathrm{d} \phi \mathrm{e}^{j(m-n) \phi} P(\overrightarrow{\mathbf{N}}, \overrightarrow{\boldsymbol{\Omega}} ; \overrightarrow{\mathbf{a}})\right] I_{m, n}(\rho)$.

The quantity in the square brackets is the input sensitivity (combined) $\mathcal{P}^{-m+n}(\overrightarrow{\mathbf{N}}, \rho ; \overrightarrow{\mathbf{a}})$ defined in Table 4 . Using Eq. (12a) in the same manner, I get the second intensity POAM expansion

$I(\overrightarrow{\mathbf{N}} ; \overrightarrow{\mathbf{a}})=\int \mathrm{d}^{2} \Omega P(\overrightarrow{\mathbf{N}}, \overrightarrow{\boldsymbol{\Omega}} ; \overrightarrow{\mathbf{a}}) \sum_{m=-\infty}^{\infty} \mathcal{I}_{m}(\rho) \mathrm{e}^{-j m \phi}=\sum_{m=-\infty}^{\infty} 2 \pi \int_{0}^{\infty} \mathrm{d} \rho \rho\left[\frac{1}{2 \pi} \int_{0}^{2 \pi} \mathrm{d} \phi \mathrm{e}^{j m \phi} P(\overrightarrow{\mathbf{N}}, \overrightarrow{\boldsymbol{\Omega}} ; \overrightarrow{\mathbf{a}})\right] \mathcal{I}_{m}(\rho)$,

where the quantity in the square brackets is the input sensitivity (combined) $\mathcal{P}^{-m}(\overrightarrow{\mathbf{N}}, \rho ; \overrightarrow{\mathbf{a}})$ defined in Table 4

The third intensity POAM expansion is obtained by substituting the output DF sensitivity expansion (Table 2) into Eq. (24b)

$$
\begin{aligned}
I(\overrightarrow{\mathbf{N}} ; \overrightarrow{\mathbf{a}}) & =\int \mathrm{d}^{2} \Omega \sum_{p=-\infty}^{\infty} D_{p}(N, \overrightarrow{\mathbf{\Omega}} ; \overrightarrow{\mathbf{a}}) \mathrm{e}^{j p v} \sum_{q=-\infty}^{\infty} D_{q}^{*}(N, \overrightarrow{\mathbf{\Omega}} ; \overrightarrow{\mathbf{a}}) \mathrm{e}^{-j q v} I(\overrightarrow{\boldsymbol{\Omega}}) \\
& =\sum_{p=-\infty}^{\infty} \sum_{q=-\infty}^{\infty}\left\{\int \mathrm{d}^{2} \Omega\left[D_{p}(N, \overrightarrow{\boldsymbol{\Omega}} ; \overrightarrow{\mathbf{a}}) D_{q}^{*}(N, \overrightarrow{\boldsymbol{\Omega}} ; \overrightarrow{\mathbf{a}})\right] I(\overrightarrow{\boldsymbol{\Omega}})\right\} \mathrm{e}^{j(p-q) v},
\end{aligned}
$$

where quantity within the curly brackets is the specific-plane correlation $I_{p, q}(N)$, and the quantity within the square brackets is the output sensitivity (separate) $P_{p, q}(N, \overrightarrow{\mathbf{\Omega}} ; \overrightarrow{\mathbf{a}})$ defined in Table 4 . To obtain the fourth intensity POAM expansion, I substitute Eq. (24b) back into Eqs. (12a) and (12b)

$I(\overrightarrow{\mathbf{N}} ; \overrightarrow{\mathbf{a}})=\sum_{p=-\infty}^{\infty} \frac{1}{2 \pi} \int_{0}^{2 \pi} \mathrm{d} v^{\prime} \mathrm{e}^{-j p v^{\prime}} \int \mathrm{d} d^{2} \Omega P\left(\overrightarrow{\mathbf{N}^{\prime}}, \overrightarrow{\boldsymbol{\Omega}} ; \overrightarrow{\mathbf{a}}\right) I(\overrightarrow{\boldsymbol{\Omega}}) \mathrm{e}^{j p v}=\sum_{p=-\infty}^{\infty}\left\{d^{2} \Omega\left[\frac{1}{2 \pi} \int_{0}^{2 \pi} \mathrm{d} v^{\prime} \mathrm{e}^{-j p v^{\prime}} P\left(\overrightarrow{\mathbf{N}^{\prime}}, \overrightarrow{\boldsymbol{\Omega}} ; \overrightarrow{\mathbf{a}}\right)\right] I(\overrightarrow{\mathbf{\Omega}})\right\} \mathrm{e}^{j p v}$

The quantity within the curly brackets is the specific-plane rancor $\mathcal{I}_{p}(N)$, and the quantity in the square brackets is the output sensitivity (combined) $\mathcal{P}_{p}(N, \overrightarrow{\mathbf{\Omega}} ; \overrightarrow{\mathbf{a}})$ defined in Table 4 .

The fifth intensity POAM expansion may be obtained by substituting the DF output sensitivity expansion (Table 2) into Eq. (C.2a),

$$
\begin{aligned}
I(\overrightarrow{\mathbf{N}} ; \overrightarrow{\mathbf{a}}) & =\sum_{m=-\infty}^{\infty} \sum_{n=-\infty}^{\infty} 2 \pi \int_{0}^{\infty} \mathrm{d} \rho \rho \frac{1}{2 \pi} \int_{0}^{2 \pi} \mathrm{d} \phi \mathrm{e}^{j(m-n) \phi} \sum_{p=-\infty}^{\infty} D_{p}(N, \rho ; \overrightarrow{\mathbf{a}}) \mathrm{e}^{j p v} \sum_{q=-\infty}^{\infty} D_{q}^{*}(N, \rho ; \overrightarrow{\mathbf{a}}) \mathrm{e}^{-j q v} I_{m, n}(\rho) \\
& =\sum_{p=-\infty}^{\infty} \sum_{q=-\infty}^{\infty}\left\{\sum_{m=-\infty}^{\infty} \sum_{n=-\infty}^{\infty} 2 \pi \int_{0}^{\infty} \mathrm{d} \rho \rho\left[\frac{1}{2 \pi} \int_{0}^{2 \pi} \mathrm{d} \phi \mathrm{e}^{j(m-n) \phi} D_{p}(N, \overrightarrow{\mathbf{\Omega}} ; \overrightarrow{\mathbf{a}}) D_{q}^{*}(N, \overrightarrow{\boldsymbol{\Omega}} ; \overrightarrow{\mathbf{a}})\right] I_{m, n}(\rho)\right\} \mathrm{e}^{j(p-q) v},
\end{aligned}
$$


where the quantity in the curly brackets is the specific-plane correlation $I_{p, q}(N ; \overrightarrow{\mathbf{a}})$, and the quantity in the square brackets is the input/output gain (combined \#2) $\mathcal{P}_{p, q}^{-m+n}(N, \rho ; \overrightarrow{\mathbf{a}})$ defined in Table 4. I obtain the sixth intensity POAM expansion in the same manner by substituting the DF output sensitivity expansion (Table 2) into Eq. (C.2b),

$$
\begin{aligned}
I(\overrightarrow{\mathbf{N}} ; \overrightarrow{\mathbf{a}}) & =\sum_{m=-\infty}^{\infty} 2 \pi \int_{0}^{\infty} \mathrm{d} \rho \rho \frac{1}{2 \pi} \int_{0}^{2 \pi} \mathrm{d} \phi \mathrm{e}^{j m \phi} \sum_{p=-\infty}^{\infty} D_{p}(N, \rho ; \overrightarrow{\mathbf{a}}) \mathrm{e}^{j p v} \sum_{q=-\infty}^{\infty} D_{q}^{*}(N, \rho ; \overrightarrow{\mathbf{a}}) \mathrm{e}^{-j q v} \mathcal{I}_{m}(\rho) \\
& =\sum_{p=-\infty}^{\infty} \sum_{q=-\infty}^{\infty}\left\{\sum_{m=-\infty}^{\infty} 2 \pi \int_{0}^{\infty} \mathrm{d} \rho \rho\left[\frac{1}{2 \pi} \int_{0}^{2 \pi} \mathrm{d} \phi \mathrm{e}^{j m \phi} D_{p}(N, \overrightarrow{\boldsymbol{\Omega}} ; \overrightarrow{\mathbf{a}}) D_{q}^{*}(N, \overrightarrow{\boldsymbol{\Omega}} ; \overrightarrow{\mathbf{a}})\right] \mathcal{I}_{m}(\rho)\right\} \mathrm{e}^{j(p-q) v},
\end{aligned}
$$

where the quantity in the curly brackets is the specific-plane correlation $I_{p, q}(N ; \overrightarrow{\mathbf{a}})$, and the quantity in the square brackets is the input/output gain (combined \#2) $\mathcal{P}_{p, q}^{-m}(N, \rho ; \overrightarrow{\mathbf{a}})$ defined in Table 4 . To obtain the seventh intensity POAM expansion, I substitute Eq. (C.2a) back into Eqs. (12a) and (12b),

$$
\begin{aligned}
I(\overrightarrow{\mathbf{N}} ; \overrightarrow{\mathbf{a}}) & =\sum_{p=-\infty}^{\infty} \frac{1}{2 \pi} \int_{0}^{2 \pi} \mathrm{d} v^{\prime} \mathrm{e}^{-j p v^{\prime}} \sum_{m=-\infty}^{\infty} \sum_{n=-\infty}^{\infty} 2 \pi \int_{0}^{\infty} \mathrm{d} \rho \rho \mathcal{P}^{-m+n}\left(\overrightarrow{\mathbf{N}^{\prime}}, \rho ; \overrightarrow{\mathbf{a}}\right) I_{m, n}(\rho) \mathrm{e}^{j p v} \\
& =\sum_{p=-\infty}^{\infty}\left\{\sum_{m=-\infty}^{\infty} \sum_{n=-\infty}^{\infty} 2 \pi \int_{0}^{\infty} \mathrm{d} \rho \rho\left[\frac{1}{2 \pi} \int_{0}^{2 \pi} \mathrm{d} v^{\prime} \mathrm{e}^{-j p v^{\prime}} \mathcal{P}^{-m+n}\left(\overrightarrow{\mathbf{N}^{\prime}}, \rho ; \overrightarrow{\mathbf{a}}\right)\right] I_{m, n}(\rho)\right\} \mathrm{e}^{j p v},
\end{aligned}
$$

where the quantity in the curly brackets is the specific-plane rancor $\mathcal{I}_{p}(N ; \overrightarrow{\mathbf{a}})$, and the quantity in the square brackets is the input/output gain (combined \#2) $\mathcal{P}_{p}^{-m+n}(N, \rho ; \overrightarrow{\mathbf{a}})$ defined in Table 4 . The last intensity POAM expansion is obtained in the same manner by substituting Eq. (C.2b) back into Eqs. (12a) and (12b),

$$
\begin{aligned}
I(\overrightarrow{\mathbf{N}} ; \overrightarrow{\mathbf{a}}) & =\sum_{p=-\infty}^{\infty} \frac{1}{2 \pi} \int_{0}^{2 \pi} \mathrm{d} v^{\prime} \mathrm{e}^{-j p v^{\prime}} \sum_{m=-\infty}^{\infty} 2 \pi \int_{0}^{\infty} \mathrm{d} \rho \rho \mathcal{P}^{-m}\left(\overrightarrow{\mathbf{N}}^{\prime}, \rho ; \overrightarrow{\mathbf{a}}\right) \mathcal{I}_{m}(\rho) \mathrm{e}^{j p v} \\
& =\sum_{p=-\infty}^{\infty}\left\{\sum_{m=-\infty}^{\infty} 2 \pi \int_{0}^{\infty} \mathrm{d} \rho \rho\left[\frac{1}{2 \pi} \int_{0}^{2 \pi} \mathrm{d} v^{\prime} \mathrm{e}^{-j p v^{\prime}} \mathcal{P}^{-m}\left(\overrightarrow{\mathbf{N}^{\prime}}, \rho ; \overrightarrow{\mathbf{a}}\right)\right] \mathcal{I}_{m}(\rho)\right\} \mathrm{e}^{j p v}
\end{aligned}
$$

where the quantity in the curly brackets is the specific-plane rancor $\mathcal{I}_{p}(N ; \overrightarrow{\mathbf{a}})$, and the quantity in the square brackets is the input/output gain (combined \#1) $\mathcal{P}_{p}^{-m}(N, \rho ; \overrightarrow{\mathbf{a}})$ defined in Table 4.

\section{Appendix D: Propagating the intensity from the celestial sphere}

Assume that the electric field in the specific plane has the form

$E(\overrightarrow{\mathbf{N}} ; \overrightarrow{\mathbf{a}}, t)=\int \mathrm{d}^{2} \Omega K(\overrightarrow{\mathbf{N}}, \overrightarrow{\boldsymbol{\Omega}} ; \overrightarrow{\mathbf{a}}) E(\overrightarrow{\boldsymbol{\Omega}} ; t)$,

where $K(\overrightarrow{\mathbf{N}}, \overrightarrow{\mathbf{\Omega}} ; \overrightarrow{\mathbf{a}})$ is the propagation kernel. The corresponding intensity is

$I(\overrightarrow{\mathbf{N}} ; \overrightarrow{\mathbf{a}})=\left\langle\frac{1}{2}|E(\overrightarrow{\mathbf{N}} ; \overrightarrow{\mathbf{a}}, t)|^{2}\right\rangle=\int \mathrm{d}^{2} \Omega \int \mathrm{d}^{2} \Omega^{\prime} K(\overrightarrow{\mathbf{N}}, \overrightarrow{\mathbf{\Omega}} ; \overrightarrow{\mathbf{a}}) K^{*}\left(\overrightarrow{\mathbf{N}}, \overrightarrow{\mathbf{\Omega}^{\prime}} ; \overrightarrow{\mathbf{a}}\right) I\left(\overrightarrow{\mathbf{\Omega}}, \overrightarrow{\mathbf{\Omega}^{\prime}}\right)$,

where

$I\left(\overrightarrow{\boldsymbol{\Omega}}, \overrightarrow{\mathbf{\Omega}^{\prime}}\right)=\left\langle\frac{1}{2} E(\overrightarrow{\boldsymbol{\Omega}} ; t) E^{*}\left(\overrightarrow{\mathbf{\Omega}^{\prime}} ; t\right)\right\rangle$

is the spatial coherence on the celestial sphere. If the electric fields on the celestial sphere are spatially incoherent over the time average, or

$\left\langle\frac{1}{2} E(\overrightarrow{\mathbf{\Omega}} ; t) E^{*}\left(\overrightarrow{\boldsymbol{\Omega}^{\prime}} ; t\right)\right\rangle=I(\overrightarrow{\mathbf{\Omega}}) \delta\left(\overrightarrow{\mathbf{\Omega}}-\overrightarrow{\mathbf{\Omega}^{\prime}}\right)$,

then Eq. (D.2a) becomes

$I(\overrightarrow{\mathbf{N}} ; \overrightarrow{\mathbf{a}})=\int \mathrm{d}^{2} \Omega|K(\overrightarrow{\mathbf{N}}, \overrightarrow{\boldsymbol{\Omega}} ; \overrightarrow{\mathbf{a}})|^{2} I(\overrightarrow{\boldsymbol{\Omega}})$.

Note that the only time that a PSF can be defined is when the electric field on the celestial sphere is spatially incoherent. For example, when propagating a spatially incoherent electric field from the celestial sphere to the observation plane (Sect. 5), $K(\overrightarrow{\mathbf{N}}, \overrightarrow{\boldsymbol{\Omega}} ; \overrightarrow{\mathbf{a}}) \rightarrow$ $D(\overrightarrow{\mathbf{R}}, \overrightarrow{\boldsymbol{\Omega}})=\mathrm{e}^{j 2 \pi k \overrightarrow{\mathbf{R}} \cdot \overrightarrow{\mathbf{s}}_{x y}(\overrightarrow{\mathbf{\Omega}})}$ and $|K(\overrightarrow{\mathbf{N}}, \overrightarrow{\boldsymbol{\Omega}} ; \overrightarrow{\mathbf{a}})|^{2} \rightarrow P(\overrightarrow{\mathbf{R}}, \overrightarrow{\boldsymbol{\Omega}})=1$. Also, when propagating a spatially incoherent electric field from the celestial sphere to the telescope image plane (Sect. 6), $K(\overrightarrow{\mathbf{N}}, \overrightarrow{\boldsymbol{\Omega}} ; \overrightarrow{\mathbf{a}}) \rightarrow D(\overrightarrow{\mathbf{r}}, \overrightarrow{\boldsymbol{\Omega}})$ and $|K(\overrightarrow{\mathbf{N}}, \overrightarrow{\boldsymbol{\Omega}} ; \overrightarrow{\mathbf{a}})|^{2} \rightarrow P(\overrightarrow{\mathbf{r}}, \overrightarrow{\boldsymbol{\Omega}})$. 


\section{Appendix E: Propagating quantities through free space}

When 9a is substituted into Eq. (17c), I obtain

$E(\overrightarrow{\mathbf{R}} ; t)=\sum_{m=-\infty}^{\infty} 2 \pi \int_{0}^{\infty} \mathrm{d} \rho \rho\left[\frac{1}{2 \pi} \int_{0}^{2 \pi} \mathrm{d} \phi \mathrm{e}^{j 2 \pi \kappa R \rho \cos (\phi-\psi)} \mathrm{e}^{j m \phi}\right] E_{m}(\rho ; t)$.

After the variable change $\chi=\phi-\psi$, the quantity in square brackets becomes

$\frac{1}{2 \pi} \int_{0-\psi}^{2 \pi-\psi} \mathrm{d} \chi \mathrm{e}^{j 2 \pi \kappa R \rho \cos \chi} \mathrm{e}^{j m \chi}=j^{m} J_{m}(2 \pi \kappa R \rho) \mathrm{e}^{j m \psi}$.

Note that the integration range is $2 \pi$ and the kernel function periodic in $2 \pi$, which means that the $-\psi$ in the integration limits may be ignored. Therefore, Eq. (E.1a) becomes

$E(\overrightarrow{\mathbf{R}} ; t)=\sum_{m=-\infty}^{\infty} E_{m}(R ; t) \mathrm{e}^{j m \psi}=\sum_{m=-\infty}^{\infty}\left\{j^{m}\left[2 \pi \int_{0}^{\infty} \mathrm{d} \rho \rho J_{m}(2 \pi \kappa R \rho) E_{m}(\rho ; t)\right]\right\} \mathrm{e}^{j m \psi}$.

The quantity in square brackets is the $m$ th order Hankel function $H\left[E_{m}(\rho ; t) ; R\right]$, arising from diffraction. Note that each source state $E_{m}(\rho ; t)$ leads only one state in the observation plane $E_{m}(R ; t)$ (the quantity in the curly brackets), which means that propagation through free space applies no external torque to the wavefronts.

The POAM expansion of the observation-plane intensity of a spatially incoherent source may be derived in a number of ways. According to Eq. (12b), a rancored intensity is the sum of correlated states with the same rancor. When this equation is substituted into Eqs. (10a) and (20), I obtain

$$
\begin{aligned}
I(\overrightarrow{\mathbf{R}}) & =\sum_{m=-\infty}^{\infty} \sum_{n=-\infty}^{\infty}\left\{j^{m-n} 2 \pi \int_{0}^{\infty} \mathrm{d} \rho \rho J_{m}(2 \pi \kappa R \rho) J_{n}(2 \pi \kappa R \rho) \sum_{k=-\infty}^{\infty} I_{k, k-(m-n)}(\rho)\right\} \mathrm{e}^{j(m-n) \psi} \\
& =\sum_{k=-\infty}^{\infty} \sum_{l=-\infty}^{\infty}\left\{j^{k-l} 2 \pi \int_{0}^{\infty} \mathrm{d} \rho \rho\left[\sum_{m=-\infty}^{\infty} J_{m}(2 \pi \kappa R \rho) J_{m-(k-l)}(2 \pi \kappa R \rho)\right] I_{k, l}(\rho)\right\} \mathrm{e}^{j(k-l) \psi} .
\end{aligned}
$$

Each term in the curly brackets is $I_{m, n}(R)$, and each of them arises from only a single correlation on the celestial sphere. But, the quantity in square brackets is the Kronecker delta function $\delta_{k, l}$, which means that

$I(\overrightarrow{\mathbf{R}})=\sum_{k=-\infty}^{\infty} 2 \pi \int_{0}^{\infty} \mathrm{d} \rho \rho I_{k, k}(\rho)$.

\section{Appendix F: Propagating quantities through an unaberrated telescope}

The DF (Eq. (23b)) of an unaberrated telescope is

$D(\overrightarrow{\mathbf{r}}, \overrightarrow{\boldsymbol{\Omega}}) \rightarrow \mathbf{D}(\overrightarrow{\mathbf{r}}, \overrightarrow{\boldsymbol{\Omega}})=\frac{1}{A_{\mathrm{ep}}} \int \mathrm{d}^{2} \mathcal{R} \mathrm{e}^{-j 2 \pi \kappa M\left[\frac{\overrightarrow{\mathbf{r}}}{\Theta}-\overrightarrow{\mathbf{s}}_{x y}(\overrightarrow{\boldsymbol{\Omega}})\right] \cdot \overrightarrow{\mathcal{R}}} \mathcal{D}_{0}=\frac{1}{A_{\mathrm{ep}}} 2 \pi \int_{0}^{R_{\mathrm{ep}}} \mathrm{d} \mathcal{R} \mathcal{R}\left[\frac{1}{2 \pi} \int_{0}^{2 \pi} \mathrm{d} \psi \mathrm{e}^{-j 2 \pi \kappa M \Gamma(\overrightarrow{\mathbf{r}}, \overrightarrow{\boldsymbol{\Omega}}) \mathcal{R} \cos [\psi-\gamma(\overrightarrow{\mathbf{r}}, \overrightarrow{\boldsymbol{\Omega}})]}\right] \mathcal{D}_{0}$,

where $\mathcal{D}_{0}$ is a real constant. With the changes of variables $\chi=\psi-\gamma(\overrightarrow{\mathbf{r}}, \overrightarrow{\mathbf{\Omega}})$ and $u=\mathcal{R} / R_{\mathrm{ep}}$, I obtain

$\mathbf{D}(\overrightarrow{\mathbf{r}}, \overrightarrow{\boldsymbol{\Omega}})=2 \int_{0}^{1} \mathrm{~d} u u\left[\frac{1}{2 \pi} \int_{0-\gamma(\overrightarrow{\mathbf{r}}, \overrightarrow{\mathbf{\Omega}})}^{2 \pi-\gamma(\overrightarrow{\mathbf{r}}, \overrightarrow{\mathbf{\Omega}})} \mathrm{d} \chi \mathrm{e}^{-j 2 \pi \kappa R_{\mathrm{tel}} \Gamma(\overrightarrow{\mathbf{r}}, \overrightarrow{\boldsymbol{\Omega}}) u \cos \chi}\right] \mathcal{D}_{0}$

The $\gamma(\overrightarrow{\mathbf{r}}, \overrightarrow{\boldsymbol{\Omega}})$ term in both integral limits may be ignored since the integrand is periodic in $2 \pi$. The quantity in the square brackets is $J_{0}\left[2 \pi \kappa R_{\mathrm{tel}} \Gamma(\overrightarrow{\mathbf{r}}, \overrightarrow{\boldsymbol{\Omega}}) u\right]$. I now apply another change of variables $v=2 \pi \kappa R_{\mathrm{tel}} \Gamma(\overrightarrow{\mathbf{r}}, \overrightarrow{\boldsymbol{\Omega}})$, which means that

$\mathbf{D}(\overrightarrow{\mathbf{r}}, \overrightarrow{\boldsymbol{\Omega}})=\frac{2}{\left[2 \pi \kappa R_{\mathrm{tel}} \Gamma(\overrightarrow{\mathbf{r}}, \overrightarrow{\boldsymbol{\Omega}}) u\right]^{2}}\left[\int_{0}^{2 \pi \kappa R_{\mathrm{tel}} \Gamma(\overrightarrow{\mathbf{r}}, \overrightarrow{\mathbf{\Omega}})} \mathrm{d} v v J_{0}(v)\right] \mathcal{D}_{0}$

The indefinite integral is $v J_{1}(v)$, so Eq. (F.1a) becomes

$\mathbf{D}(\overrightarrow{\mathbf{r}}, \overrightarrow{\boldsymbol{\Omega}})=2 \frac{J_{1}\left[2 \pi \kappa R_{\mathrm{tel}} \Gamma(\overrightarrow{\mathbf{r}}, \overrightarrow{\boldsymbol{\Omega}})\right]}{2 \pi \kappa R_{\mathrm{tel}} \Gamma(\overrightarrow{\mathbf{r}}, \overrightarrow{\mathbf{\Omega}})} \mathcal{D}_{0}=\mathcal{D}_{0} \mathcal{J}_{1}\left[2 \pi \kappa R_{\mathrm{tel}} \Gamma(\overrightarrow{\mathbf{r}}, \overrightarrow{\mathbf{\Omega}})\right]$ 
The unaberrated PSF is just the square of this DF,

$$
P(\overrightarrow{\mathbf{r}}, \overrightarrow{\mathbf{\Omega}}) \rightarrow \mathbf{P}(\overrightarrow{\mathbf{r}}, \overrightarrow{\mathbf{\Omega}})=\mathbf{D}^{2}(\overrightarrow{\mathbf{r}}, \overrightarrow{\mathbf{\Omega}})=\mathcal{P}_{0} \mathcal{A}\left[2 \pi \kappa R_{\mathrm{tel}} \Gamma(\overrightarrow{\mathbf{r}}, \overrightarrow{\mathbf{\Omega}})\right]
$$

Deriving the principal DF POAM expansions (Eq. (27a)) is relatively straightforward. I begin with the input/output gains of the unaberrated DFs (Table 2),

$$
\begin{aligned}
D_{p}^{-m}(r, \rho) & =\frac{1}{2 \pi} \int_{0-\psi}^{2 \pi-\psi} \mathrm{d} \phi \mathrm{e}^{j m \phi} \int_{0-\psi}^{2 \pi-\psi} \mathrm{d} \xi \mathrm{e}^{-j p \xi} \frac{1}{A_{\mathrm{ep}}} \int \mathrm{d}^{2} \mathcal{R} \mathrm{e}^{-j 2 \pi \kappa M\left[\frac{\overrightarrow{\mathbf{r}}}{\Theta}-\overrightarrow{\mathbf{s}}_{x y}(\overrightarrow{\boldsymbol{\Omega}})\right] \cdot \overrightarrow{\mathcal{R}}} \mathcal{D}_{0} \\
& =\mathcal{D}_{0} 2 \int_{0}^{1} \mathrm{~d} u u\left[\frac{1}{2 \pi} \int_{0}^{2 \pi} \mathrm{d} \gamma \mathrm{e}^{j p \gamma} \mathrm{e}^{-j 2 \pi \kappa \frac{R_{\mathrm{tel}}}{\Theta} u \cos \gamma}\right]\left[\frac{1}{2 \pi} \int_{0}^{2 \pi} \mathrm{d} \chi \mathrm{e}^{j m \chi} \mathrm{e}^{-j 2 \pi \kappa R_{\mathrm{te}} \rho u \cos \chi}\right]^{*}\left[\frac{1}{2 \pi} \int_{0}^{2 \pi} \mathrm{d} \psi \mathrm{e}^{-j(m-p) \psi}\right],
\end{aligned}
$$

where I made the variable substitutions $\gamma=\xi-\psi, \chi=\phi-\psi$, and $u=\mathcal{R} / R_{\mathrm{ep}}$. The $\psi$ variable has been removed from the integration limits because the integrands are periodic in $2 \pi$. The rightmost quantity in square brackets is $\delta_{m, p}$. Using the Bessel function identity of Eq. (E.1b) to replace the other two quantities in square brackets, I obtain the principal DF POAM expansions. With the same equations, it is then possible to derive the input and output sensitivities

$D^{-m}(\overrightarrow{\mathbf{r}}, \rho)=\sum_{p=-\infty}^{\infty} D_{p}^{-m}(r, \rho) \mathrm{e}^{j p \xi}=\mathbf{D}(r, \rho ; m) \mathrm{e}^{j m \xi}$

and

$D_{p}(r, \overrightarrow{\boldsymbol{\Omega}})=\sum_{m=-\infty}^{\infty} D_{p}^{-m}(r, \rho) \mathrm{e}^{-j m \phi}=\mathbf{D}(r, \rho ; p) \mathrm{e}^{-j p \phi}$.

Deriving principal PSF POAM expansions takes a little more effort. I start with the input/output (correlated/correlated) expansion in Table 3. For an unaberrated instrument, the image-plane correlations become

$$
\begin{aligned}
I_{p, q}(r) & =\sum_{m=-\infty}^{\infty} \sum_{n=-\infty}^{\infty} 2 \pi \int_{0}^{\infty} \mathrm{d} \rho \rho \mathcal{P}_{p, q}^{-m+n}(r, \rho) I_{m, n}(\rho)=\sum_{m=-\infty}^{\infty} \sum_{n=-\infty}^{\infty} 2 \pi \int_{0}^{\infty} \mathrm{d} \rho \rho\left[\frac{1}{2 \pi} \int_{0}^{2 \pi} \mathrm{d} \phi \mathrm{e}^{j(m-n-p+q) \phi}\right] \mathbf{D}(r, \rho ; p) \mathbf{D}(r, \rho ; q) I_{m, n}(\rho) \\
& =2 \pi \int_{0}^{\infty} \mathrm{d} \rho \rho \mathbf{P}(r, \rho ; p, q) \mathcal{I}_{p-q}(\rho)
\end{aligned}
$$

which proves Eq. (28a). If I turn this equation into a relationship between the rancors of the image plane and the celestial sphere, then

$\mathcal{I}_{m}(r)=\sum_{p=-\infty}^{\infty} I_{p, p-m}(r)=2 \pi \int_{0}^{\infty} \mathrm{d} \rho \rho\left[\sum_{p=-\infty}^{\infty} \mathbf{P}(r, \rho ; p, p-m)\right] \mathcal{I}_{m}(\rho)=2 \pi \int_{0}^{\infty} \mathrm{d} \rho \rho \mathbf{P}(r, \rho ; m) \mathcal{I}_{m}(\rho)$,

which proves Eq. (28b).

To prove that the integrated POAM probabilities are conserved between the celestial sphere and the image plane for an unaberrated telescope, I begin with the relationship between correlations on the celestial sphere and correlations in the image plane (Eq. (28a)),

$I_{p, p}(r)=2 \pi \int_{0}^{\infty} \mathrm{d} \rho \rho \mathbf{P}(r, \rho ; 0) I_{p, p}(\rho)$.

When this equation is normalized by the total intensity in the image plane

$I^{i p}=\int \mathrm{d}^{2} r I(\overrightarrow{\mathbf{r}})=\int \mathrm{d}^{2} \Omega\left[\int \mathrm{d}^{2} r \mathbf{P}(\overrightarrow{\mathbf{r}}, \overrightarrow{\boldsymbol{\Omega}})\right] I(\overrightarrow{\boldsymbol{\Omega}})=\int \mathrm{d}^{2} \Omega P(\overrightarrow{\boldsymbol{\Omega}}) I(\overrightarrow{\boldsymbol{\Omega}})$

and rearranged, I obtain the integrated probability in the image plane (Eq. (14b)),

$$
\begin{aligned}
p_{p, p}^{i p} & =\frac{I_{p, p}^{i p}}{I^{i p}}=\frac{2 \pi \int_{0}^{\infty} \mathrm{d} r r I_{p, p}(r)}{I^{i p}}=2 \pi \int_{0}^{\infty} \mathrm{d} r r p_{p, p}(r) \\
& =2 \pi \int_{0}^{\infty} \mathrm{d} \rho \rho\left[\frac{I^{\mathrm{cs}}}{I^{i p}} 2 \pi \int_{0}^{\infty} \mathrm{d} r r \mathbf{P}(r, \rho ; 0)\right] p_{p, p}(\rho)=2 \pi \int_{0}^{\infty} \mathrm{d} \rho \rho\left[\frac{I^{\mathrm{cs}}}{I^{i p}} 2 \pi \int_{0}^{\infty} \mathrm{d} r r \sum_{k=-\infty}^{\infty} \mathbf{P}(r, \rho ; k, k)\right] p_{p, p}(\rho),
\end{aligned}
$$

where I have also normalized the celestial sphere correlation by $I^{\mathrm{cs}}$ to form the radial probability density on the celestial sphere. I let the radial integration limit go to infinity, which will be well approximated by a detector that captures the light from several of the inner rings of the Airy disk. For Eq. (F.5c), which has a form similar to Eq. (14b), to be equal to the integrated probability on the celestial sphere $p_{p, p}^{\mathrm{cs}}($ Eq. $(21 \mathrm{~b}))$, I must prove that the quantity within the square brackets is unity. 
The sum in Eq. (F.5c) may be derived from the following equation,

$$
\begin{aligned}
\frac{1}{2 \pi} \int_{0}^{2 \pi} \mathrm{d} \phi \frac{1}{2 \pi} \int_{0}^{2 \pi} \mathrm{d} \xi P(\overrightarrow{\mathbf{r}}, \overrightarrow{\boldsymbol{\Omega}}) & =\frac{1}{2 \pi} \int_{0}^{2 \pi} \mathrm{d} \phi \frac{1}{2 \pi} \int_{0}^{2 \pi} \mathrm{d} \xi|D(\overrightarrow{\mathbf{r}}, \overrightarrow{\boldsymbol{\Omega}})|^{2} \\
& =\sum_{p=-\infty}^{\infty} \sum_{q=-\infty}^{\infty} \sum_{k=-\infty}^{\infty} \sum_{l=-\infty}^{\infty} D_{p}^{-k}(r, \rho) D_{q}^{-l, *}(r, \rho)\left[\frac{1}{2 \pi} \int_{0}^{2 \pi} \mathrm{d} \phi \mathrm{e}^{j(p-q) \phi}\right]\left[\frac{1}{2 \pi} \int_{0}^{2 \pi} \mathrm{d} \xi \mathrm{e}^{-j(k-l) \xi}\right] \\
& =\sum_{p=-\infty}^{\infty} \sum_{k=-\infty}^{\infty}\left|D_{p}^{-k}(r, \rho)\right|^{2}=\sum_{k=-\infty}^{\infty}|\mathbf{D}(r, \rho ; k)|^{2}=\sum_{k=-\infty}^{\infty} \mathbf{P}(r, \rho ; k, k)
\end{aligned}
$$

Substituting the first line of this equation into the square backets of Eq. (F.5c) and rearranging, I find that

$\frac{I^{\mathrm{cs}}}{I^{i p}} 2 \pi \int_{0}^{\infty} \mathrm{d} r r \frac{1}{2 \pi} \int_{0}^{2 \pi} \mathrm{d} \phi \frac{1}{2 \pi} \int_{0}^{2 \pi} \mathrm{d} \xi \mathbf{P}(\overrightarrow{\mathbf{r}}, \overrightarrow{\mathbf{\Omega}})=\frac{I^{\mathrm{cs}}}{I^{\mathrm{ip}}} \frac{1}{2 \pi} \int_{0}^{2 \pi} \mathrm{d} \phi\left[\int \mathrm{d}^{2} r \mathbf{P}(\overrightarrow{\mathbf{r}}, \overrightarrow{\mathbf{\Omega}})\right]$

When integrating over the entire image plane, the quantity in the square brackets is $C \approx$ constant and the integral over $\phi$ becomes unity. According to Eqs. (F.5b), $I^{\text {ip }} \rightarrow C I^{\mathrm{cs}}$, which confirms that the integrated probabilities in the image plane and on the celestial sphere are identical. Also, since each integrated probability is conserved, the POAM expectation value is also conserved.

\section{Appendix G: Calculating the POAM expansion of a coronagraph sector mask}

Substituting Eq. (37a) into Eq. (34b) and making a simple change of variables, I find that

$$
\begin{aligned}
M_{h}(r, \overrightarrow{\boldsymbol{\Omega}}) & =\sum_{g=0}^{2 N-1}\left[\frac{1}{2 \pi} \int_{\frac{2 \pi g}{2 N}-\frac{\pi}{2 N}}^{\frac{2 \pi g}{2 N}+\frac{\pi}{2 N}} \mathrm{~d} \xi \mathrm{e}^{-j h \xi}\right] m_{g}(r, \overrightarrow{\boldsymbol{\Omega}})=\sum_{g=0}^{2 N-1}\left[\frac{1}{-j 2 \pi h} \int_{-j \frac{2 \pi h g}{2 N}+j \frac{\pi h}{2 N}}^{-j \frac{2 \pi h g}{2 N}-j \frac{\pi h}{2 N}} \mathrm{~d} u \mathrm{e}^{u}\right] m_{g}(r, \overrightarrow{\boldsymbol{\Omega}}) \\
& =\left\{\frac{1}{2 N} \sum_{g=0}^{2 N-1} \mathrm{e}^{-j 2 \pi \frac{g h}{2 N}} m_{g}(r, \overrightarrow{\boldsymbol{\Omega}})\right\} \operatorname{sinc}\left(\pi \frac{h}{2 N}\right)=\mathcal{M}_{h}(r, \overrightarrow{\boldsymbol{\Omega}}) \operatorname{sinc}\left(\pi \frac{h}{2 N}\right),
\end{aligned}
$$

where the quantity in the curly brackets is the $h$ th DFT component. The DFT components of the alternating-phase mask are obtained by substituting Eq. (39a) into Eq. (37c)

$$
\mathcal{M}_{h}(r, \overrightarrow{\boldsymbol{\Omega}}) \rightarrow \mathcal{M}_{h}=\frac{1}{2 N} \sum_{g=0}^{2 N-1} \mathrm{e}^{-j 2 \pi \frac{g h}{2 N}} \mathrm{e}^{j p \pi}=\frac{1}{2 N} \sum_{g=0}^{2 N-1} \mathrm{e}^{-j 2 \pi \frac{g(h-N)}{2 N}}
$$

The components are unity when $h$ is an odd multiple (positive or negative) of $N$, and zero otherwise. Similarly, the DFT components of the vortex mask are obtained by substituting Eq. (41a) into Eq. (37c)

$$
\mathcal{M}_{h}(r, \overrightarrow{\boldsymbol{\Omega}}) \rightarrow \mathcal{M}_{h}=\frac{1}{2 N} \sum_{g=0}^{2 N-1} \mathrm{e}^{-j 2 \pi \frac{g h}{2 N}} \mathrm{e}^{j 2 \pi \frac{g N_{w}}{2 N}}=\frac{1}{2 N} \sum_{g=0}^{2 N-1} \mathrm{e}^{-j 2 \pi \frac{g\left(h-N_{w}\right)}{2 N}}
$$

The components are unity when $h$ is $N_{w}$ plus an even mutiple (positive or negative) of $N$, and zero otherwise.

\section{Appendix H: Calculating the POAM quantities of a Michelson interferometer}

Using Eq. (46b), I calculate the IDF output sensitivity

$$
\begin{aligned}
D_{u}(B, \overrightarrow{\boldsymbol{\Omega}} ; d, \overrightarrow{\mathbf{r}}) & =\frac{1}{2 \pi} \int_{0}^{2 \pi} \mathrm{d} \beta \mathrm{e}^{-j u \beta} D(\overrightarrow{\mathbf{B}}, \overrightarrow{\boldsymbol{\Omega}} ; \mathrm{d}, \overrightarrow{\mathbf{r}}) \\
& =\left[\frac{1}{2 \pi} \int_{0}^{2 \pi} \mathrm{d} \beta \mathrm{e}^{-j u \beta} \mathrm{e}^{j 2 \pi \kappa \frac{1}{2} B \rho \cos (\beta-\phi)}\right] D_{A}(\overrightarrow{\mathbf{r}}, \overrightarrow{\boldsymbol{\Omega}}) \mathrm{e}^{-j 2 \pi \kappa \frac{1}{2} d}+\left[\frac{1}{2 \pi} \int_{0}^{2 \pi} \mathrm{d} \beta \mathrm{e}^{-j u \beta} \mathrm{e}^{-j 2 \pi \kappa \frac{1}{2} B \rho \cos (\beta-\phi)}\right] D_{B}(\overrightarrow{\mathbf{r}}, \overrightarrow{\boldsymbol{\Omega}}) \mathrm{e}^{j 2 \pi \kappa \frac{1}{2} d}
\end{aligned}
$$

The quantities in square brackets are $j^{u} J_{u}\left(2 \pi \kappa \frac{1}{2} B \rho\right) \mathrm{e}^{-j u \phi}$ and $j^{-u} J_{u}\left(2 \pi \kappa \frac{1}{2} B \rho\right) \mathrm{e}^{-j u \phi}$, respectively, which means that

$D_{u}(B, \overrightarrow{\boldsymbol{\Omega}} ; d, \overrightarrow{\mathbf{r}})=j^{u} J_{u}\left(2 \pi \kappa \frac{1}{2} B \rho\right)\left\{[E(u)+O(u)] D_{A}(\overrightarrow{\mathbf{r}}, \overrightarrow{\boldsymbol{\Omega}}) \mathrm{e}^{-j 2 \pi \kappa \frac{1}{2} d}+[E(u)-O(u)] D_{B}(\overrightarrow{\mathbf{r}}, \overrightarrow{\boldsymbol{\Omega}}) \mathrm{e}^{j 2 \pi \kappa \frac{1}{2} d}\right\} \mathrm{e}^{-j u \phi}$

The quantity in the curly brackets is $D_{\text {arm }}(\overrightarrow{\mathbf{r}}, \overrightarrow{\mathbf{\Omega}} ; d, u)$, in Eq. (50b).

According to Eqs. (50b) and (52b), the IPSF output sensitivity (separate) is

$$
P_{u, v}(B, \overrightarrow{\boldsymbol{\Omega}} ; d, \overrightarrow{\mathbf{r}})=D_{u}(B, \overrightarrow{\boldsymbol{\Omega}} ; d, \overrightarrow{\mathbf{r}}) D_{v}^{*}(B, \overrightarrow{\mathbf{\Omega}} ; d, \overrightarrow{\mathbf{r}})=j^{u-v} J_{u}\left(2 \pi \kappa \frac{1}{2} B \rho\right) J_{v}\left(2 \pi \kappa \frac{1}{2} B \rho\right) P_{\mathrm{arm}}(\overrightarrow{\mathbf{r}}, \overrightarrow{\mathbf{\Omega}} ; d, u, v) \mathrm{e}^{-j(u-v) \phi}
$$


Equations (48e-48h), (50c), and (52c), can be used to explicitly state the arm factor product

$$
\begin{aligned}
& P_{\mathrm{arm}}(\overrightarrow{\mathbf{r}}, \overrightarrow{\boldsymbol{\Omega}} ; d, u, v)=\left[P_{A A}(\overrightarrow{\mathbf{r}}, \overrightarrow{\boldsymbol{\Omega}})+P_{B B}(\overrightarrow{\mathbf{r}}, \overrightarrow{\boldsymbol{\Omega}})+2 \operatorname{Re}\left\{P_{A B}(\overrightarrow{\mathbf{r}}, \overrightarrow{\boldsymbol{\Omega}}) \mathrm{e}^{-j 2 \pi k d}\right\}\right] E(u) E(v) \\
& +\left[P_{A A}(\overrightarrow{\mathbf{r}}, \overrightarrow{\mathbf{\Omega}})-P_{B B}(\overrightarrow{\mathbf{r}}, \overrightarrow{\mathbf{\Omega}})-j 2 \operatorname{Im}\left\{P_{A B}(\overrightarrow{\mathbf{r}}, \overrightarrow{\mathbf{\Omega}}) \mathrm{e}^{-j 2 \pi \kappa d}\right\}\right] E(u) O(v) \\
& +\left[P_{A A}(\overrightarrow{\mathbf{r}}, \overrightarrow{\mathbf{\Omega}})-P_{B B}(\overrightarrow{\mathbf{r}}, \overrightarrow{\mathbf{\Omega}})+j 2 \operatorname{Im}\left\{P_{A B}(\overrightarrow{\mathbf{r}}, \overrightarrow{\mathbf{\Omega}}) \mathrm{e}^{-j 2 \pi \kappa d}\right\}\right] O(u) E(v) \\
& +\left[P_{A A}(\overrightarrow{\mathbf{r}}, \overrightarrow{\boldsymbol{\Omega}})+P_{B B}(\overrightarrow{\mathbf{r}}, \overrightarrow{\boldsymbol{\Omega}})-2 \operatorname{Re}\left\{P_{A B}(\overrightarrow{\mathbf{r}}, \overrightarrow{\boldsymbol{\Omega}}) \mathrm{e}^{-j 2 \pi \kappa d}\right\}\right] O(u) O(v) \\
& =\left(P_{0}(\overrightarrow{\mathbf{r}}, \overrightarrow{\mathbf{\Omega}})[E(u) E(v)+O(u) O(v)]+\Delta P_{0}(\overrightarrow{\mathbf{r}}, \overrightarrow{\mathbf{\Omega}})[E(u) O(v)+O(u) E(v)]\right) \\
& +\left(2 \operatorname{Re}\left\{P_{A B}(\overrightarrow{\mathbf{r}}, \overrightarrow{\mathbf{\Omega}})\right\}[E(u) E(v)-O(u) O(v)]-j 2 \operatorname{Im}\left\{P_{A B}(\overrightarrow{\mathbf{r}}, \overrightarrow{\mathbf{\Omega}})\right\}[E(u) O(v)-O(u) E(v)]\right)
\end{aligned}
$$

In the second part of this equation, the quantities in the square brackets are $E(u-v), O(u-v),(-1)^{v} E(u-v)$, and $(-1)^{v+1} O(u-v)$, respectively. Also in the second part of this equation, the first quantity in parentheses is $P_{\text {uncorr }}(\overrightarrow{\mathbf{r}}, \overrightarrow{\mathbf{\Omega}} ; u-v)($ Eq. (52d)) and the second quantity is $(-1)^{v} P_{\text {corr }}(\overrightarrow{\mathbf{r}}, \overrightarrow{\mathbf{\Omega}} ; d, u-v)$ (Eq. (52e)).

\section{Appendix I: Measuring binary star orbital parameters with a limited rancorimeter}

The COL for a binary star, expressed in Cartesian coordinates with respect to a fixed origin, is

$\alpha_{C L}=\frac{I_{1}}{I} \alpha_{1}+\frac{I_{2}}{I} \alpha_{2}=\frac{1}{1+q_{L}} \alpha_{1}+\frac{q_{L}}{1+q_{L}} \alpha_{2}$

and

$\delta_{C L}=\frac{I_{1}}{I} \delta_{1}+\frac{I_{2}}{I} \delta_{2}=\frac{1}{1+q_{L}} \delta_{1}+\frac{q_{L}}{1+q_{L}} \delta_{2}$,

where $q_{L}=I_{2} / I_{1}$ is the light ratio and $I=I_{1}+I_{2}$ is the light total. For each observation, the COL acts as the reference point,

$\alpha_{1, *}=\alpha_{1}-\alpha_{C L}=\frac{q_{L}}{1+q_{L}} \Delta \alpha=\frac{q_{L}}{1+q_{L}} \rho_{*} \cos \phi_{*}=\rho_{1, *} \cos \phi_{*}$,

$\delta_{1, *}=\delta_{1}-\delta_{C L}=\frac{q_{L}}{1+q_{L}} \Delta \delta=\frac{q_{L}}{1+q_{L}} \rho_{*} \sin \phi_{*}=\rho_{1, *} \sin \phi_{*}$,

$\alpha_{2, *}=\alpha_{2}-\alpha_{C L}=-\frac{1}{1+q_{L}} \Delta \alpha=-\frac{1}{1+q_{L}} \rho_{*} \cos \phi_{*}=-\rho_{2, *} \cos \phi_{*}$,

and

$\delta_{2, *}=\delta_{2}-\delta_{C L}=-\frac{1}{1+q_{L}} \Delta \delta=-\frac{1}{1+q_{L}} \rho_{*} \sin \phi_{*}=-\rho_{2, *} \sin \phi_{*}$.

The quantities $q_{L}, \rho_{1, *}, \rho_{2, *}$, and $\phi_{*}$ are measured with the limited rancorimeter (Sect. 9.1), but $\rho_{*}=\rho_{1, *}+\rho_{2, *}$ and $\phi_{*}$ are used for orbit fitting.

\section{Appendix J: Expressing the diffraction function perturbation using the zernike expansion}

When Eq. (76) is substituted into Eq. (75a), I obtain

$$
\begin{aligned}
\Delta D(\overrightarrow{\mathbf{r}}, \overrightarrow{\boldsymbol{\Omega}})= & j \mathcal{D}_{0} \sum_{m=0}^{\infty} \sum_{\substack{n=m, n-m=\text { even }}}^{\infty} 2 \int_{0}^{1} \mathrm{~d} u u\left[\frac{1}{2 \pi} \int_{0}^{2 \pi} \mathrm{d} \psi \mathrm{e}^{-j 2 \pi \kappa R_{\mathrm{te}} \Gamma(\overrightarrow{\mathbf{r}}, \overrightarrow{\mathbf{\Omega}}) u \cos [\psi-\gamma(\overrightarrow{\mathbf{r}}, \overrightarrow{\boldsymbol{\Omega}})]} \mathrm{e}^{j m \psi}\right] R_{m, n}(u) F_{m, n}(\overrightarrow{\boldsymbol{\Omega}}) \\
& +j \mathcal{D}_{0} \sum_{m=0}^{\infty} \sum_{\substack{n=m, n-m=\text { even }}}^{\infty} 2 \int_{0}^{1} \mathrm{~d} u u\left[\frac{1}{2 \pi} \int_{0}^{2 \pi} \mathrm{d} \psi \mathrm{e}^{-j 2 \pi \kappa R_{\mathrm{tel}} \Gamma(\overrightarrow{\mathbf{r}}, \overrightarrow{\boldsymbol{\Omega}}) u \cos [\psi-\gamma(\overrightarrow{\mathbf{r}}, \overrightarrow{\mathbf{\Omega}})]} \mathrm{e}^{-j m \psi}\right] R_{m, n}(u) F_{m, n}^{*}(\overrightarrow{\boldsymbol{\Omega}})
\end{aligned}
$$

after some rearranging. With a change of variables $\chi=\psi-\gamma(\overrightarrow{\mathbf{r}}, \overrightarrow{\mathbf{\Omega}})$, this equation becomes

$$
\begin{aligned}
\Delta D(\overrightarrow{\mathbf{r}}, \overrightarrow{\boldsymbol{\Omega}})= & j \mathcal{D}_{0} \sum_{m=0}^{\infty} \sum_{\substack{n=m, n \\
n-m=\text { even }}}^{\infty} 2 \int_{0}^{1} \mathrm{~d} u u\left[\frac{1}{2 \pi} \int_{0-\gamma(\overrightarrow{\mathbf{r}}, \overrightarrow{\mathbf{\Omega}})}^{2 \pi-\gamma(\overrightarrow{\mathbf{r}}, \overrightarrow{\boldsymbol{\Omega}})} \mathrm{d} \chi \mathrm{e}^{-j 2 \pi \kappa R_{\mathrm{tel}} \Gamma(\overrightarrow{\mathbf{r}}, \overrightarrow{\boldsymbol{\Omega}}) u \cos \chi} \mathrm{e}^{j m \psi}\right] \mathrm{e}^{j m \gamma(\overrightarrow{\mathbf{r}}, \overrightarrow{\boldsymbol{\Omega}})} R_{m, n}(u) F_{m, n}(\overrightarrow{\boldsymbol{\Omega}}) \\
& +j \mathcal{D}_{0} \sum_{m=0}^{\infty} \sum_{\substack{n=m, n-m=\text { even }}}^{\infty} 2 \int_{0}^{1} \mathrm{~d} u u\left[\frac{1}{2 \pi} \int_{0-\gamma(\overrightarrow{\mathbf{r}}, \overrightarrow{\boldsymbol{\Omega}})}^{2 \pi-\gamma(\overrightarrow{\mathbf{r}}, \overrightarrow{\mathbf{\Omega}})} \mathrm{d} \chi \mathrm{e}^{-j 2 \pi \kappa R_{\mathrm{tel}} \Gamma(\overrightarrow{\mathbf{r}}, \overrightarrow{\boldsymbol{\Omega}}) u \cos \chi} \mathrm{e}^{-j m \psi}\right] \mathrm{e}^{-j m \gamma(\overrightarrow{\mathbf{r}}, \overrightarrow{\boldsymbol{\Omega}})} R_{m, n}(u) F_{m, n}^{*}(\overrightarrow{\boldsymbol{\Omega}}) .
\end{aligned}
$$


The $\gamma(\overrightarrow{\mathbf{r}}, \overrightarrow{\boldsymbol{\Omega}})$ terms in the integral limits may be ignored, since the integrands are periodic in $2 \pi$. Using the identities

$j^{k} J_{k}(x)=\frac{1}{2 \pi} \int_{0}^{2 \pi} \mathrm{d} \chi \mathrm{e}^{-j x \cos \chi} \mathrm{e}^{j k \chi}$

and $J_{-k}(x)=(-1)^{k} J_{k}(x)$, Eq. (J.1b) becomes

$\Delta D(\overrightarrow{\mathbf{r}}, \overrightarrow{\boldsymbol{\Omega}})=j \mathcal{D}_{0} \sum_{m=0}^{\infty} \sum_{\substack{n=m, n \\ n-m=\mathrm{even}}}^{\infty} j^{-m}\left\{2 \int_{0}^{1} \mathrm{~d} u u J_{m}\left[2 \pi \kappa R_{\mathrm{tel}} \Gamma(\overrightarrow{\mathbf{r}}, \overrightarrow{\boldsymbol{\Omega}}) u\right] R_{m, n}(u)\right\}\left[F_{m, n}(\overrightarrow{\boldsymbol{\Omega}}) \mathrm{e}^{j m \gamma(\overrightarrow{\mathbf{r}}, \overrightarrow{\boldsymbol{\Omega}})}+F_{m, n}^{*}(\overrightarrow{\boldsymbol{\Omega}}) \mathrm{e}^{-j m \gamma(\overrightarrow{\mathbf{r}}, \overrightarrow{\boldsymbol{\Omega}})}\right]$.

The quantity in the curly braces is $j^{n-m} \mathcal{J}_{n+1}\left[2 \pi \kappa R_{\text {tel }} \Gamma(\overrightarrow{\mathbf{r}}, \overrightarrow{\mathbf{\Omega}})\right]$, thus giving Eq. (77).

\section{References}

Allen, L., Beijersbergen, M. W., Spreeuw, R. J. C., \& Woerdman, J. P. 1992, Phys. Rev. A, 45, 8185

Anzolin, G., Tamburini, F., Bianchini, A., Umbriaco, G., \& Barbieri, C. 2008, A\&A, 488, 1159

Bazhenov, V. Yu., Vasnetsov, M. V., \& Soskin, M. S. 1990, J. Exp. Theor. Phys. Lett., 52, 429

Boccaletti, A., Riaud, P., \& Rouan, D. 2002 PASP, 114, 132

Boccaletti, A., Riaud, P., Baudoz, P., et al. 2004, PASP, 116, 1061

Bracewell, R. 1978, Nature, 274, 780

Bracewell, R. N. 1986, The Fourier Transform \& Its Applications (New York, NY: McGraw-Hill)

Crepp, J. R., Vanden Heuvel, A. D., \& Ge, J. 2007, ApJ, 661, 1323

Danchi, W. C., Allen, R. J., Benford, D. J., et al. 2004, Proc. SPIE, 5491, 236

Elias II, N. M., Harwit, M., Leisawitz, D., \& Rinehart, S. A. 2007, ApJ, 657, 1178

Ferrari, A. 2007, ApJ, 657, 1201

Gori, F., Santarsiero, M., Borghi, R., \& Guattari, G. 1998, Eur. J. Phys., 19, 439

Griffiths, D. J. 1981, Introduction to Electrodynamics (Englewood Cliffs, NJ: Prentice-Hall)

Guyon, O., Pluzhnik, E. A., Kuchner, M. J., Collins, B., \& Ridgway, S. T. 2006, ApJS, 167, 81

Harwit, M. 2003, ApJ, 597, 1266

Heitler, W. 1984, The Quantum Theory of Radiation (New York, NY: Dover)

Indebetouw, G. 1993, J. Mod. Opt., 40, 73

Jackson, J. D. 1998, Classical Electrodynamics (New York, NY: Wiley)

Kennedy, S. A., Szabo, M. J., Teslow, H., Porterfield, J. Z., \& Abraham, E. R. I. 2002, Phys. Rev. A, 66, 043801

Leach, J., Padgett, M. J., Barnett, S. M., Franke-Arnold, S., \& Courtial, J. 2002, Phys. Rev. Lett., 88, 257901

Lee, J. H., Foo, G., Johnson, E. G., \& Swartzlander Jr., G. A. 2006, Phys. Rev. Lett., 97, 3901

Mandel, L., \& Wolf, E. 1995, Optical Coherence and Quantum Optics (Cambrige: Cambridge University Press), 491

Marcuse, D. 1980, Principles of Quantum Electronics (New York, NY: Academic)

Molina-Terriza, G., Torres, J. P., \& Torner, L. 2007, Nat. Phys., 3, 305

Padgett, M., Courtial, J., \& Allen, L. 2004, Physics Today, 57(5) 35

Reitz, J. R., Milford, F. J., \& Christy, R. W. 1980, Foundations of Electromagnetic Theory (Reading, MA: Addison-Wesley)

Riaud, P., Boccaletti, A., Rouan, D., Lemarquis, F., \& Labeyrie, A. 2001, PASP, 113, 1145

Riaud, P., Boccaletti, A., Baudrand, J., \& Rouan, D. 2003, PASP, 115, 712

Rouan, D., Riaud, P., Boccaletti, A., Clénet, Y., \& Labeyrie, A. 2000, PASP, 112, 1479

Sjöholm, J., \& Palmer, K. 2007, Diploma Thesis, Uppsala University

Tamburini, F., Anzolin, G., Umbriaco, G., Bianchini, A., \& Barbieri, C. 2006, Phys. Rev. Lett., 97, 163903

Tamm, C., \& Weiss, C. O. 1990, J. Opt. Soc. Am. B, 7, 1034

Thidé, B., Then, H., Sjöholm, J., et al. 2007, Phys. Rev. Lett., 99, 087701

Wyant, J. C., \& Creath, K. 1992, Applied Optics and Optical Engineering, Vol. XI, Chap. 1 (Burlington, MA: Academic Press)

Yao, E., Franke-Arnold, S., Courtial, J., Barnett, S., \& Padgett, M. 2006, Opt. Exp., 14, 9071 